\title{
Thorium(IV) and Uranium(IV) Ketimide Complexes Prepared by Nitrile Insertion into Actinide-Alkyl and -Aryl Bonds
}

\author{
Kimberly C. Jantunen, Carol J. Burns, Ingrid Castro-Rodriguez, Ryan E. Da Re, Jeffery T. Golden, \\ David E. Morris, Brian L. Scott, Felicia L. Taw, and Jaqueline L. Kiplinger*
}

\section{$\underline{\text { SUPPORTING INFORMATION }}$}

Crystallographic information (tables with bond lengths, bond angles, atomic coordinates, and anisotropic displacement parameters) for complexes 9b (Tables S1-S5), 9c (Tables S6-S10), 10a (Tables S11-S15), 10b (Tables S16-S20), 11b (Tables S21-S25), 11c (Tables S26-S30), and 12b (Tables S31-S35). 
Table S1. Crystal data and structure refinement for $\left(\mathrm{C}_{5} \mathrm{Me}_{5}\right)_{2} \mathrm{Th}\left(\mathrm{CH}_{2} \mathrm{Ph}\right)_{2}(\mathbf{9 b})$.

Identification code

Empirical formula

Formula weight

Temperature

Wavelength

Crystal system

Space group

Unit cell dimensions

Volume

$\mathrm{Z}$

Density (calculated)

Absorption coefficient

$\mathrm{F}(000)$

Crystal size

Theta range for data collection

Index ranges

Reflections collected

Independent reflections

Completeness to theta $=25.43^{\circ}$

Max. and min. transmission

Refinement method

Data / restraints / parameters

Goodness-of-fit on $\mathrm{F}^{2}$

Final R indices [I $>2 \operatorname{sigma}(\mathrm{I})]$

$\mathrm{R}$ indices (all data)

Largest diff. peak and hole $\operatorname{ccd} 783$

C34 H44 Th

684.73

203(2) K

$0.71073 \AA$

monoclinic

P $2{ }_{1} / \mathrm{n}$

$\mathrm{a}=9.886(3) \AA$

$\alpha=90^{\circ}$.

$\mathrm{b}=12.410(4) \AA$

$\beta=100.639(5)^{\circ}$.

$\mathrm{c}=24.078(7) \AA$

$\gamma=90^{\circ}$.

2903.1(14) $\AA^{3}$

4

$1.567 \mathrm{Mg} / \mathrm{m}^{3}$

$5.156 \mathrm{~mm}^{-1}$

1352

$0.28 \times 0.10 \times 0.10 \mathrm{~mm}^{3}$

1.72 to $25.43^{\circ}$.

$-11<=\mathrm{h}<=11,-14<=\mathrm{k}<=13,-28<=1<=29$

18073

$5312[\mathrm{R}(\mathrm{int})=0.0328]$

$99.3 \%$

0.6266 and 0.3262

Full-matrix least-squares on $\mathrm{F}^{2}$

$5312 / 0 / 316$

1.058

$\mathrm{R} 1=0.0404, \mathrm{wR} 2=0.1286$

$\mathrm{R} 1=0.0512, \mathrm{wR} 2=0.1343$

2.038 and -1.154 e. $\AA^{-3}$ 
Table S2. Atomic coordinates ( $\left.\mathrm{x} 10^{4}\right)$ and equivalent isotropic displacement parameters $\left(\AA^{2} \times 10^{3}\right)$ for $\left(\mathrm{C}_{5} \mathrm{Me}_{5}\right)_{2} \mathrm{Th}\left(\mathrm{CH}_{2} \mathrm{Ph}\right)_{2}(9 \mathbf{b})$. U(eq) is defined as one third of the trace of the orthogonalized $\mathrm{U}^{\mathrm{ij}}$ tensor.

\begin{tabular}{|c|c|c|c|c|}
\hline & $\mathrm{x}$ & $\mathrm{y}$ & z & $\mathrm{U}(\mathrm{eq})$ \\
\hline $\operatorname{Th}(1)$ & 3063(1) & 2318(1) & $1137(1)$ & $23(1)$ \\
\hline$C(1)$ & 5122(9) & $3192(7)$ & $563(3)$ & $37(2)$ \\
\hline$C(2)$ & 5797(8) & $3079(7)$ & $1129(4)$ & $38(2)$ \\
\hline$C(3)$ & $5199(8)$ & $3805(7)$ & 1474(4) & $34(2)$ \\
\hline$C(4)$ & 4168(8) & 4394(6) & $1106(3)$ & $33(2)$ \\
\hline$C(5)$ & $4100(8)$ & 3996(6) & $545(3)$ & $30(2)$ \\
\hline$C(6)$ & $5533(13)$ & 2725(8) & $44(5)$ & $59(3)$ \\
\hline$C(7)$ & 7073(10) & 2411(8) & 1352(6) & $53(3)$ \\
\hline $\mathrm{C}(8)$ & $5719(10)$ & 4060(8) & 2083(4) & $46(2)$ \\
\hline$C(9)$ & $3528(10)$ & $5407(7)$ & 1290(4) & $44(2)$ \\
\hline$C(10)$ & $3179(11)$ & $4422(8)$ & 14(3) & $51(3)$ \\
\hline$C(11)$ & $625(8)$ & 1949(8) & $1590(4)$ & $36(2)$ \\
\hline $\mathrm{C}(12)$ & $834(8)$ & $3078(8)$ & 1603(3) & $35(2)$ \\
\hline$C(13)$ & $549(8)$ & $3456(7)$ & 1035(3) & $31(2)$ \\
\hline$C(14)$ & $224(8)$ & 2573(6) & $676(3)$ & $25(2)$ \\
\hline$C(15)$ & $248(7)$ & 1631(7) & 1020(4) & $35(2)$ \\
\hline$C(16)$ & $553(10)$ & $1268(10)$ & 2098(5) & $60(3)$ \\
\hline$C(17)$ & $1089(10)$ & 3782(9) & $2115(4)$ & $52(3)$ \\
\hline $\mathrm{C}(18)$ & 292(9) & 4619(8) & $852(4)$ & $47(2)$ \\
\hline$C(19)$ & $-290(11)$ & 2612(8) & $47(4)$ & $48(2)$ \\
\hline$C(20)$ & $-355(10)$ & $542(8)$ & $808(5)$ & $59(3)$ \\
\hline$C(21)$ & $2880(9)$ & 1051(7) & $292(3)$ & $33(2)$ \\
\hline $\mathrm{C}(22)$ & $3632(8)$ & 202(7) & $642(3)$ & $31(2)$ \\
\hline$C(23)$ & 2971(9) & $-571(7)$ & $919(4)$ & $39(2)$ \\
\hline $\mathrm{C}(24)$ & $3716(10)$ & $-1317(8)$ & $1285(4)$ & $49(2)$ \\
\hline$C(25)$ & $5095(10)$ & $-1323(8)$ & $1397(4)$ & $50(2)$ \\
\hline$C(26)$ & $5760(9)$ & $-581(8)$ & $1118(4)$ & $45(2)$ \\
\hline$C(27)$ & $5084(9)$ & 167(7) & 754(4) & $40(2)$ \\
\hline$C(28)$ & $4096(8)$ & $1273(7)$ & $2027(3)$ & $31(2)$ \\
\hline$C(29)$ & 4849(8) & 1602(6) & $2583(3)$ & $31(2)$ \\
\hline $\mathrm{C}(30)$ & $6229(9)$ & $1342(8)$ & 2764(4) & $43(2)$ \\
\hline
\end{tabular}




$\begin{array}{lllll}\mathrm{C}(31) & 6970(10) & 1665(9) & 3284(4) & 52(3) \\ \mathrm{C}(32) & 6337(13) & 2271(8) & 3648(4) & 54(3) \\ \mathrm{C}(33) & 4956(14) & 2518(8) & 3486(5) & 52(3) \\ \mathrm{C}(34) & 4236(10) & 2200(7) & 2965(4) & 40(2)\end{array}$


Table S3. Bond lengths $[\AA]$ and angles $\left[^{\circ}\right]$ for $\left(\mathrm{C}_{5} \mathrm{Me}_{5}\right)_{2} \mathrm{Th}\left(\mathrm{CH}_{2} \mathrm{Ph}\right)_{2}(\mathbf{9 b})$.

\begin{tabular}{|c|c|}
\hline $\operatorname{Th}(1)-C(28)$ & $2.551(7)$ \\
\hline $\operatorname{Th}(1)-C(21)$ & $2.552(7)$ \\
\hline $\operatorname{Th}(1)-\mathrm{C}(4)$ & $2.805(8)$ \\
\hline $\operatorname{Th}(1)-\mathrm{C}(3)$ & $2.809(8)$ \\
\hline $\operatorname{Th}(1)-C(12)$ & $2.814(8)$ \\
\hline $\operatorname{Th}(1)-C(5)$ & $2.822(7)$ \\
\hline $\operatorname{Th}(1)-C(13)$ & $2.829(8)$ \\
\hline $\operatorname{Th}(1)-C(14)$ & $2.838(8)$ \\
\hline $\operatorname{Th}(1)-C(11)$ & $2.859(7)$ \\
\hline $\operatorname{Th}(1)-C(2)$ & $2.867(8)$ \\
\hline $\operatorname{Th}(1)-C(15)$ & $2.874(7)$ \\
\hline $\operatorname{Th}(1)-C(1)$ & $2.876(8)$ \\
\hline$C(1)-C(2)$ & $1.410(13)$ \\
\hline$C(1)-C(5)$ & $1.415(12)$ \\
\hline$C(1)-C(6)$ & $1.500(12)$ \\
\hline$C(2)-C(3)$ & $1.426(12)$ \\
\hline$C(2)-C(7)$ & $1.522(13)$ \\
\hline$C(3)-C(4)$ & $1.423(11)$ \\
\hline$C(3)-C(8)$ & $1.495(11)$ \\
\hline$C(4)-C(5)$ & $1.429(11)$ \\
\hline$C(4)-C(9)$ & $1.510(12)$ \\
\hline$C(5)-C(10)$ & $1.521(11)$ \\
\hline$C(11)-C(15)$ & $1.412(12)$ \\
\hline$C(11)-C(12)$ & $1.416(14)$ \\
\hline$C(11)-C(16)$ & $1.500(12)$ \\
\hline$C(12)-C(13)$ & $1.426(11)$ \\
\hline$C(12)-C(17)$ & $1.494(11)$ \\
\hline$C(13)-C(14)$ & $1.395(11)$ \\
\hline$C(13)-C(18)$ & $1.516(12)$ \\
\hline$C(14)-C(15)$ & $1.429(12)$ \\
\hline$C(14)-C(19)$ & $1.507(12)$ \\
\hline$C(15)-C(20)$ & $1.527(13)$ \\
\hline$C(21)-C(22)$ & $1.464(12)$ \\
\hline$C(22)-C(23)$ & $1.398(12)$ \\
\hline
\end{tabular}




\begin{tabular}{|c|c|}
\hline$C(22)-C(27)$ & $1.411(12)$ \\
\hline$C(23)-C(24)$ & $1.391(14)$ \\
\hline$C(24)-C(25)$ & $1.340(14)$ \\
\hline$C(25)-C(26)$ & $1.377(14)$ \\
\hline$C(26)-C(27)$ & $1.364(13)$ \\
\hline$C(28)-C(29)$ & $1.464(11)$ \\
\hline $\mathrm{C}(29)-\mathrm{C}(30)$ & $1.392(11)$ \\
\hline$C(29)-C(34)$ & $1.403(12)$ \\
\hline $\mathrm{C}(30)-\mathrm{C}(31)$ & $1.387(12)$ \\
\hline $\mathrm{C}(31)-\mathrm{C}(32)$ & $1.389(16)$ \\
\hline $\mathrm{C}(32)-\mathrm{C}(33)$ & $1.384(19)$ \\
\hline $\mathrm{C}(33)-\mathrm{C}(34)$ & $1.382(14)$ \\
\hline $\mathrm{C}(28)-\mathrm{Th}(1)-\mathrm{C}(21)$ & $108.4(3)$ \\
\hline $\mathrm{C}(28)-\mathrm{Th}(1)-\mathrm{C}(4)$ & $113.1(3)$ \\
\hline $\mathrm{C}(21)-\mathrm{Th}(1)-\mathrm{C}(4)$ & $121.0(2)$ \\
\hline $\mathrm{C}(28)-\operatorname{Th}(1)-\mathrm{C}(3)$ & $85.9(3)$ \\
\hline $\mathrm{C}(21)-\mathrm{Th}(1)-\mathrm{C}(3)$ & $125.0(3)$ \\
\hline $\mathrm{C}(4)-\mathrm{Th}(1)-\mathrm{C}(3)$ & $29.4(2)$ \\
\hline $\mathrm{C}(28)-\mathrm{Th}(1)-\mathrm{C}(12)$ & $93.3(2)$ \\
\hline $\mathrm{C}(21)-\mathrm{Th}(1)-\mathrm{C}(12)$ & $125.4(3)$ \\
\hline $\mathrm{C}(4)-\mathrm{Th}(1)-\mathrm{C}(12)$ & $92.3(3)$ \\
\hline $\mathrm{C}(3)-\mathrm{Th}(1)-\mathrm{C}(12)$ & $105.5(3)$ \\
\hline $\mathrm{C}(28)-\mathrm{Th}(1)-\mathrm{C}(5)$ & $132.3(2)$ \\
\hline $\mathrm{C}(21)-\mathrm{Th}(1)-\mathrm{C}(5)$ & $91.7(2)$ \\
\hline $\mathrm{C}(4)-\mathrm{Th}(1)-\mathrm{C}(5)$ & $29.4(2)$ \\
\hline $\mathrm{C}(3)-\mathrm{Th}(1)-\mathrm{C}(5)$ & $48.5(2)$ \\
\hline $\mathrm{C}(12)-\operatorname{Th}(1)-\mathrm{C}(5)$ & $109.8(3)$ \\
\hline $\mathrm{C}(28)-\mathrm{Th}(1)-\mathrm{C}(13)$ & $122.4(2)$ \\
\hline $\mathrm{C}(21)-\mathrm{Th}(1)-\mathrm{C}(13)$ & $107.8(3)$ \\
\hline $\mathrm{C}(4)-\operatorname{Th}(1)-\mathrm{C}(13)$ & $83.1(2)$ \\
\hline $\mathrm{C}(3)-\mathrm{Th}(1)-\mathrm{C}(13)$ & $107.4(2)$ \\
\hline $\mathrm{C}(12)-\mathrm{Th}(1)-\mathrm{C}(13)$ & $29.3(2)$ \\
\hline$C(5)-\operatorname{Th}(1)-C(13)$ & $89.0(2)$ \\
\hline $\mathrm{C}(28)-\mathrm{Th}(1)-\mathrm{C}(14)$ & $126.8(2)$ \\
\hline $\mathrm{C}(21)-\mathrm{Th}(1)-\mathrm{C}(14)$ & $80.7(2)$ \\
\hline
\end{tabular}




\begin{tabular}{|c|c|}
\hline $\mathrm{C}(4)-\mathrm{Th}(1)-\mathrm{C}(14)$ & $104.4(2)$ \\
\hline $\mathrm{C}(3)-\mathrm{Th}(1)-\mathrm{C}(14)$ & $132.5(2)$ \\
\hline $\mathrm{C}(12)-\mathrm{Th}(1)-\mathrm{C}(14)$ & $47.8(2)$ \\
\hline $\mathrm{C}(5)-\mathrm{Th}(1)-\mathrm{C}(14)$ & $98.3(2)$ \\
\hline $\mathrm{C}(13)-\mathrm{Th}(1)-\mathrm{C}(14)$ & $28.5(2)$ \\
\hline $\mathrm{C}(28)-\mathrm{Th}(1)-\mathrm{C}(11)$ & $80.4(2)$ \\
\hline $\mathrm{C}(21)-\mathrm{Th}(1)-\mathrm{C}(11)$ & $105.4(3)$ \\
\hline $\mathrm{C}(4)-\mathrm{Th}(1)-\mathrm{C}(11)$ & 121.1(3) \\
\hline $\mathrm{C}(3)-\mathrm{Th}(1)-\mathrm{C}(11)$ & $129.5(3)$ \\
\hline $\mathrm{C}(12)-\mathrm{Th}(1)-\mathrm{C}(11)$ & $28.9(3)$ \\
\hline $\mathrm{C}(5)-\mathrm{Th}(1)-\mathrm{C}(11)$ & $136.2(2)$ \\
\hline $\mathrm{C}(13)-\mathrm{Th}(1)-\mathrm{C}(11)$ & $47.5(2)$ \\
\hline $\mathrm{C}(14)-\mathrm{Th}(1)-\mathrm{C}(11)$ & $47.5(2)$ \\
\hline $\mathrm{C}(28)-\mathrm{Th}(1)-\mathrm{C}(2)$ & $86.6(3)$ \\
\hline $\mathrm{C}(21)-\mathrm{Th}(1)-\mathrm{C}(2)$ & $97.2(3)$ \\
\hline $\mathrm{C}(4)-\mathrm{Th}(1)-\mathrm{C}(2)$ & $47.5(2)$ \\
\hline $\mathrm{C}(3)-\mathrm{Th}(1)-\mathrm{C}(2)$ & $29.1(2)$ \\
\hline $\mathrm{C}(12)-\mathrm{Th}(1)-\mathrm{C}(2)$ & $134.5(3)$ \\
\hline $\mathrm{C}(5)-\mathrm{Th}(1)-\mathrm{C}(2)$ & $47.3(3)$ \\
\hline $\mathrm{C}(13)-\mathrm{Th}(1)-\mathrm{C}(2)$ & $130.5(2)$ \\
\hline $\mathrm{C}(14)-\mathrm{Th}(1)-\mathrm{C}(2)$ & $145.7(2)$ \\
\hline $\mathrm{C}(11)-\mathrm{Th}(1)-\mathrm{C}(2)$ & $156.6(3)$ \\
\hline $\mathrm{C}(28)-\mathrm{Th}(1)-\mathrm{C}(15)$ & $99.4(3)$ \\
\hline $\mathrm{C}(21)-\mathrm{Th}(1)-\mathrm{C}(15)$ & $79.3(3)$ \\
\hline$C(4)-T h(1)-C(15)$ & $130.1(2)$ \\
\hline $\mathrm{C}(3)-\mathrm{Th}(1)-\mathrm{C}(15)$ & $152.4(2)$ \\
\hline $\mathrm{C}(12)-\mathrm{Th}(1)-\mathrm{C}(15)$ & $47.4(3)$ \\
\hline $\mathrm{C}(5)-\mathrm{Th}(1)-\mathrm{C}(15)$ & $127.2(2)$ \\
\hline $\mathrm{C}(13)-\mathrm{Th}(1)-\mathrm{C}(15)$ & $47.2(3)$ \\
\hline $\mathrm{C}(14)-\mathrm{Th}(1)-\mathrm{C}(15)$ & $29.0(2)$ \\
\hline $\mathrm{C}(11)-\mathrm{Th}(1)-\mathrm{C}(15)$ & $28.5(2)$ \\
\hline $\mathrm{C}(2)-\mathrm{Th}(1)-\mathrm{C}(15)$ & $173.8(3)$ \\
\hline $\mathrm{C}(28)-\mathrm{Th}(1)-\mathrm{C}(1)$ & $112.7(3)$ \\
\hline$C(21)-\operatorname{Th}(1)-C(1)$ & $78.5(2)$ \\
\hline $\mathrm{C}(4)-\mathrm{Th}(1)-\mathrm{C}(1)$ & $47.7(2)$ \\
\hline $\mathrm{C}(3)-\mathrm{Th}(1)-\mathrm{C}(1)$ & $47.9(2)$ \\
\hline
\end{tabular}




\begin{tabular}{|c|c|}
\hline $\mathrm{C}(12)-\mathrm{Th}(1)-\mathrm{C}(1)$ & $137.8(3)$ \\
\hline $\mathrm{C}(5)-\mathrm{Th}(1)-\mathrm{C}(1)$ & $28.7(2)$ \\
\hline $\mathrm{C}(13)-\mathrm{Th}(1)-\mathrm{C}(1)$ & $117.2(3)$ \\
\hline $\mathrm{C}(14)-\mathrm{Th}(1)-\mathrm{C}(1)$ & $120.4(2)$ \\
\hline $\mathrm{C}(11)-\mathrm{Th}(1)-\mathrm{C}(1)$ & $164.8(3)$ \\
\hline $\mathrm{C}(2)-\mathrm{Th}(1)-\mathrm{C}(1)$ & $28.4(3)$ \\
\hline $\mathrm{C}(15)-\mathrm{Th}(1)-\mathrm{C}(1)$ & $145.4(3)$ \\
\hline $\mathrm{C}(2)-\mathrm{C}(1)-\mathrm{C}(5)$ & $107.9(7)$ \\
\hline $\mathrm{C}(2)-\mathrm{C}(1)-\mathrm{C}(6)$ & $127.9(9)$ \\
\hline$C(5)-C(1)-C(6)$ & $123.2(9)$ \\
\hline $\mathrm{C}(2)-\mathrm{C}(1)-\mathrm{Th}(1)$ & $75.4(5)$ \\
\hline $\mathrm{C}(5)-\mathrm{C}(1)-\mathrm{Th}(1)$ & $73.5(4)$ \\
\hline $\mathrm{C}(6)-\mathrm{C}(1)-\mathrm{Th}(1)$ & $125.4(6)$ \\
\hline $\mathrm{C}(1)-\mathrm{C}(2)-\mathrm{C}(3)$ & 109.1(8) \\
\hline $\mathrm{C}(1)-\mathrm{C}(2)-\mathrm{C}(7)$ & $127.5(9)$ \\
\hline $\mathrm{C}(3)-\mathrm{C}(2)-\mathrm{C}(7)$ & $123.0(9)$ \\
\hline $\mathrm{C}(1)-\mathrm{C}(2)-\mathrm{Th}(1)$ & $76.1(5)$ \\
\hline $\mathrm{C}(3)-\mathrm{C}(2)-\mathrm{Th}(1)$ & $73.2(4)$ \\
\hline$C(7)-C(2)-T h(1)$ & $122.6(6)$ \\
\hline $\mathrm{C}(4)-\mathrm{C}(3)-\mathrm{C}(2)$ & $106.8(8)$ \\
\hline $\mathrm{C}(4)-\mathrm{C}(3)-\mathrm{C}(8)$ & $125.4(8)$ \\
\hline $\mathrm{C}(2)-\mathrm{C}(3)-\mathrm{C}(8)$ & $126.9(8)$ \\
\hline $\mathrm{C}(4)-\mathrm{C}(3)-\operatorname{Th}(1)$ & $75.2(4)$ \\
\hline $\mathrm{C}(2)-\mathrm{C}(3)-\mathrm{Th}(1)$ & $77.7(5)$ \\
\hline $\mathrm{C}(8)-\mathrm{C}(3)-\mathrm{Th}(1)$ & $121.7(6)$ \\
\hline$C(3)-C(4)-C(5)$ & $108.4(7)$ \\
\hline $\mathrm{C}(3)-\mathrm{C}(4)-\mathrm{C}(9)$ & $122.5(8)$ \\
\hline$C(5)-C(4)-C(9)$ & $128.0(8)$ \\
\hline $\mathrm{C}(3)-\mathrm{C}(4)-\mathrm{Th}(1)$ & $75.5(5)$ \\
\hline $\mathrm{C}(5)-\mathrm{C}(4)-\operatorname{Th}(1)$ & $76.0(4)$ \\
\hline $\mathrm{C}(9)-\mathrm{C}(4)-\mathrm{Th}(1)$ & $124.8(6)$ \\
\hline$C(1)-C(5)-C(4)$ & $107.8(7)$ \\
\hline $\mathrm{C}(1)-\mathrm{C}(5)-\mathrm{C}(10)$ & $125.8(7)$ \\
\hline $\mathrm{C}(4)-\mathrm{C}(5)-\mathrm{C}(10)$ & $126.3(8)$ \\
\hline $\mathrm{C}(1)-\mathrm{C}(5)-\mathrm{Th}(1)$ & $77.7(5)$ \\
\hline$C(4)-C(5)-T h(1)$ & $74.6(4)$ \\
\hline
\end{tabular}




\begin{tabular}{|c|c|}
\hline$C(10)-C(5)-\operatorname{Th}(1)$ & $117.6(5)$ \\
\hline $\mathrm{C}(15)-\mathrm{C}(11)-\mathrm{C}(12)$ & $108.1(7)$ \\
\hline$C(15)-C(11)-C(16)$ & $126.3(9)$ \\
\hline $\mathrm{C}(12)-\mathrm{C}(11)-\mathrm{C}(16)$ & $124.6(9)$ \\
\hline$C(15)-C(11)-T h(1)$ & $76.3(4)$ \\
\hline $\mathrm{C}(12)-\mathrm{C}(11)-\mathrm{Th}(1)$ & $73.8(4)$ \\
\hline $\mathrm{C}(16)-\mathrm{C}(11)-\mathrm{Th}(1)$ & $124.9(5)$ \\
\hline $\mathrm{C}(11)-\mathrm{C}(12)-\mathrm{C}(13)$ & $107.6(7)$ \\
\hline $\mathrm{C}(11)-\mathrm{C}(12)-\mathrm{C}(17)$ & $126.8(8)$ \\
\hline$C(13)-C(12)-C(17)$ & $125.0(9)$ \\
\hline $\mathrm{C}(11)-\mathrm{C}(12)-\mathrm{Th}(1)$ & $77.3(4)$ \\
\hline$C(13)-C(12)-T h(1)$ & $76.0(4)$ \\
\hline $\mathrm{C}(17)-\mathrm{C}(12)-\mathrm{Th}(1)$ & $120.0(6)$ \\
\hline$C(14)-C(13)-C(12)$ & $108.5(7)$ \\
\hline$C(14)-C(13)-C(18)$ & $124.2(8)$ \\
\hline $\mathrm{C}(12)-\mathrm{C}(13)-\mathrm{C}(18)$ & $125.8(8)$ \\
\hline $\mathrm{C}(14)-\mathrm{C}(13)-\mathrm{Th}(1)$ & $76.1(5)$ \\
\hline $\mathrm{C}(12)-\mathrm{C}(13)-\mathrm{Th}(1)$ & $74.8(4)$ \\
\hline $\mathrm{C}(18)-\mathrm{C}(13)-\mathrm{Th}(1)$ & $126.8(5)$ \\
\hline$C(13)-C(14)-C(15)$ & $107.9(7)$ \\
\hline$C(13)-C(14)-C(19)$ & $126.4(7)$ \\
\hline$C(15)-C(14)-C(19)$ & $125.0(8)$ \\
\hline $\mathrm{C}(13)-\mathrm{C}(14)-\mathrm{Th}(1)$ & $75.4(5)$ \\
\hline$C(15)-C(14)-T h(1)$ & $76.9(4)$ \\
\hline$C(19)-C(14)-T h(1)$ & $121.5(6)$ \\
\hline $\mathrm{C}(11)-\mathrm{C}(15)-\mathrm{C}(14)$ & $107.9(7)$ \\
\hline$C(11)-C(15)-C(20)$ & $126.1(9)$ \\
\hline $\mathrm{C}(14)-\mathrm{C}(15)-\mathrm{C}(20)$ & $124.6(8)$ \\
\hline $\mathrm{C}(11)-\mathrm{C}(15)-\mathrm{Th}(1)$ & $75.2(4)$ \\
\hline $\mathrm{C}(14)-\mathrm{C}(15)-\mathrm{Th}(1)$ & $74.1(4)$ \\
\hline $\mathrm{C}(20)-\mathrm{C}(15)-\mathrm{Th}(1)$ & $127.3(6)$ \\
\hline $\mathrm{C}(22)-\mathrm{C}(21)-\mathrm{Th}(1)$ & $91.7(4)$ \\
\hline $\mathrm{C}(23)-\mathrm{C}(22)-\mathrm{C}(27)$ & $115.9(8)$ \\
\hline$C(23)-C(22)-C(21)$ & $122.5(8)$ \\
\hline$C(27)-C(22)-C(21)$ & $121.5(8)$ \\
\hline$C(23)-C(22)-T h(1)$ & $106.0(5)$ \\
\hline
\end{tabular}




\begin{tabular}{lr}
$\mathrm{C}(27)-\mathrm{C}(22)-\mathrm{Th}(1)$ & $102.2(5)$ \\
$\mathrm{C}(21)-\mathrm{C}(22)-\mathrm{Th}(1)$ & $58.9(4)$ \\
$\mathrm{C}(24)-\mathrm{C}(23)-\mathrm{C}(22)$ & $121.3(8)$ \\
$\mathrm{C}(25)-\mathrm{C}(24)-\mathrm{C}(23)$ & $122.1(9)$ \\
$\mathrm{C}(24)-\mathrm{C}(25)-\mathrm{C}(26)$ & $117.2(9)$ \\
$\mathrm{C}(27)-\mathrm{C}(26)-\mathrm{C}(25)$ & $123.2(9)$ \\
$\mathrm{C}(26)-\mathrm{C}(27)-\mathrm{C}(22)$ & $120.3(8)$ \\
$\mathrm{C}(29)-\mathrm{C}(28)-\mathrm{Th}(1)$ & $133.0(6)$ \\
$\mathrm{C}(30)-\mathrm{C}(29)-\mathrm{C}(34)$ & $115.8(8)$ \\
$\mathrm{C}(30)-\mathrm{C}(29)-\mathrm{C}(28)$ & $121.7(7)$ \\
$\mathrm{C}(34)-\mathrm{C}(29)-\mathrm{C}(28)$ & $122.6(7)$ \\
$\mathrm{C}(31)-\mathrm{C}(30)-\mathrm{C}(29)$ & $122.6(9)$ \\
$\mathrm{C}(30)-\mathrm{C}(31)-\mathrm{C}(32)$ & $120.1(9)$ \\
$\mathrm{C}(33)-\mathrm{C}(32)-\mathrm{C}(31)$ & $118.6(9)$ \\
$\mathrm{C}(34)-\mathrm{C}(33)-\mathrm{C}(32)$ & $120.6(10)$ \\
$\mathrm{C}(33)-\mathrm{C}(34)-\mathrm{C}(29)$ & $122.3(9)$ \\
& \\
\hline
\end{tabular}

Symmetry transformations used to generate equivalent atoms: 
Table S4. Anisotropic displacement parameters $\left(\AA^{2} \mathrm{x} 10^{3}\right)$ for $\left(\mathrm{C}_{5} \mathrm{Me}_{5}\right)_{2} \mathrm{Th}\left(\mathrm{CH}_{2} \mathrm{Ph}\right)_{2}$ (9b). The anisotropic displacement factor exponent takes the form: $-2 \pi^{2}\left[h^{2} a^{* 2} U^{11}+\ldots+2 h k a^{*} b^{*} U^{12}\right]$

\begin{tabular}{|c|c|c|c|c|c|c|}
\hline & $\mathrm{U}^{11}$ & $\mathrm{U}^{22}$ & $\mathrm{U}^{33}$ & $\mathrm{U}^{23}$ & $\mathrm{U}^{13}$ & $\mathrm{U}^{12}$ \\
\hline $\operatorname{Th}(1)$ & 21(1) & $24(1)$ & $25(1)$ & $-2(1)$ & $5(1)$ & $-1(1)$ \\
\hline$C(1)$ & $45(5)$ & $34(5)$ & $35(4)$ & $-8(3)$ & $20(4)$ & $-11(4)$ \\
\hline$C(2)$ & $25(4)$ & $27(4)$ & $67(6)$ & $-3(4)$ & $18(4)$ & $-2(3)$ \\
\hline$C(3)$ & $21(4)$ & $32(5)$ & $50(5)$ & $-2(4)$ & $3(3)$ & $0(3)$ \\
\hline$C(4)$ & $32(4)$ & $27(4)$ & $41(5)$ & $-5(3)$ & 11(3) & $-8(3)$ \\
\hline$C(5)$ & $41(5)$ & $26(4)$ & $25(4)$ & 1(3) & $10(3)$ & $-12(3)$ \\
\hline$C(6)$ & $73(8)$ & $55(7)$ & $62(7)$ & $-14(5)$ & $44(6)$ & $-17(5)$ \\
\hline$C(7)$ & $27(5)$ & $45(6)$ & $89(8)$ & $-2(5)$ & $16(5)$ & $5(4)$ \\
\hline$C(8)$ & $45(5)$ & $46(6)$ & $43(5)$ & $-3(4)$ & $-3(4)$ & $-10(4)$ \\
\hline$C(9)$ & $46(5)$ & $31(5)$ & $55(6)$ & $-5(4)$ & $9(4)$ & 1(4) \\
\hline$C(10)$ & $72(7)$ & $57(6)$ & $19(4)$ & $4(4)$ & $-5(4)$ & $-18(5)$ \\
\hline $\mathrm{C}(11)$ & $23(4)$ & $40(5)$ & $48(5)$ & $12(4)$ & $16(3)$ & $5(4)$ \\
\hline$C(12)$ & $28(4)$ & $52(5)$ & $26(4)$ & $-5(4)$ & $6(3)$ & $13(4)$ \\
\hline$C(13)$ & $23(4)$ & $35(5)$ & $35(4)$ & $3(3)$ & $6(3)$ & $3(3)$ \\
\hline$C(14)$ & $22(4)$ & $36(4)$ & $18(3)$ & $-2(3)$ & $4(3)$ & $4(3)$ \\
\hline$C(15)$ & $13(4)$ & $42(5)$ & $53(5)$ & $-5(4)$ & $10(3)$ & $-4(3)$ \\
\hline$C(16)$ & $36(5)$ & $81(8)$ & $69(7)$ & $41(6)$ & $24(5)$ & $17(5)$ \\
\hline$C(17)$ & $45(6)$ & $78(8)$ & $30(5)$ & $-15(4)$ & $0(4)$ & $13(5)$ \\
\hline$C(18)$ & $33(5)$ & $43(6)$ & $63(6)$ & $6(4)$ & $3(4)$ & $12(4)$ \\
\hline$C(19)$ & $37(5)$ & $69(7)$ & $34(5)$ & $-5(4)$ & $0(4)$ & $2(4)$ \\
\hline$C(20)$ & $29(5)$ & $50(6)$ & $103(9)$ & $-17(6)$ & $23(5)$ & $-13(4)$ \\
\hline $\mathrm{C}(21)$ & $41(5)$ & $43(5)$ & $17(4)$ & $-9(3)$ & $7(3)$ & $-9(4)$ \\
\hline$C(22)$ & $38(5)$ & $35(5)$ & $23(4)$ & $-13(3)$ & $12(3)$ & $-3(3)$ \\
\hline$C(23)$ & $31(5)$ & $45(5)$ & $42(5)$ & $-2(4)$ & $8(4)$ & $-9(4)$ \\
\hline$C(24)$ & $58(6)$ & $42(6)$ & $55(6)$ & $-2(4)$ & $27(5)$ & $-9(5)$ \\
\hline$C(25)$ & $52(6)$ & $42(6)$ & $53(6)$ & $2(4)$ & $6(5)$ & $5(4)$ \\
\hline$C(26)$ & $34(5)$ & $54(6)$ & $48(5)$ & $-6(4)$ & 11(4) & $4(4)$ \\
\hline$C(27)$ & $37(5)$ & $44(5)$ & $41(5)$ & $-7(4)$ & $16(4)$ & $-3(4)$ \\
\hline$C(28)$ & $29(4)$ & $34(5)$ & $31(4)$ & $4(3)$ & $9(3)$ & $10(3)$ \\
\hline$C(29)$ & $29(4)$ & $28(4)$ & $36(4)$ & $5(3)$ & $6(3)$ & 2(3) \\
\hline
\end{tabular}




\begin{tabular}{lllllll}
$\mathrm{C}(30)$ & $43(5)$ & $49(6)$ & $36(5)$ & $0(4)$ & $3(4)$ & $4(4)$ \\
$\mathrm{C}(31)$ & $38(5)$ & $62(7)$ & $51(6)$ & $11(5)$ & $-9(4)$ & $-7(5)$ \\
$\mathrm{C}(32)$ & $61(7)$ & $56(7)$ & $37(5)$ & $-3(4)$ & $-9(5)$ & $-14(5)$ \\
$\mathrm{C}(33)$ & $69(8)$ & $48(6)$ & $41(6)$ & $-7(4)$ & $14(5)$ & $1(5)$ \\
$\mathrm{C}(34)$ & $38(5)$ & $38(5)$ & $43(5)$ & $-5(4)$ & $3(4)$ & $6(4)$ \\
\hline
\end{tabular}


Table S5. Hydrogen coordinates ( $\left.x 10^{4}\right)$ and isotropic displacement parameters $\left(\AA^{2} \times 10^{3}\right)$ for $\left(\mathrm{C}_{5} \mathrm{Me}_{5}\right)_{2} \mathrm{Th}\left(\mathrm{CH}_{2} \mathrm{Ph}\right)_{2}(\mathbf{9 b})$.

\begin{tabular}{|c|c|c|c|c|}
\hline & $\mathrm{x}$ & $\mathrm{y}$ & z & $\mathrm{U}(\mathrm{eq})$ \\
\hline $\mathrm{H}(6 \mathrm{~A})$ & 4873 & 2930 & -283 & 89 \\
\hline $\mathrm{H}(6 \mathrm{~B})$ & 5565 & 1953 & 74 & 89 \\
\hline $\mathrm{H}(6 \mathrm{C})$ & 6425 & 2992 & 10 & 89 \\
\hline $\mathrm{H}(7 \mathrm{~A})$ & 7311 & 2489 & 1755 & 79 \\
\hline $\mathrm{H}(7 \mathrm{~B})$ & 7824 & 2655 & 1183 & 79 \\
\hline $\mathrm{H}(7 \mathrm{C})$ & 6890 & 1666 & 1260 & 79 \\
\hline $\mathrm{H}(8 \mathrm{~A})$ & 6411 & 3545 & 2239 & 69 \\
\hline $\mathrm{H}(8 \mathrm{~B})$ & 4971 & 4026 & 2287 & 69 \\
\hline $\mathrm{H}(8 \mathrm{C})$ & 6108 & 4771 & 2115 & 69 \\
\hline $\mathrm{H}(9 \mathrm{~A})$ & 2861 & 5680 & 981 & 66 \\
\hline $\mathrm{H}(9 \mathrm{~B})$ & 4231 & 5939 & 1402 & 66 \\
\hline $\mathrm{H}(9 \mathrm{C})$ & 3085 & 5246 & 1603 & 66 \\
\hline $\mathrm{H}(10 \mathrm{~A})$ & 2578 & 4966 & 115 & 77 \\
\hline $\mathrm{H}(10 \mathrm{~B})$ & 2639 & 3842 & -176 & 77 \\
\hline $\mathrm{H}(10 \mathrm{C})$ & 3737 & 4727 & -233 & 77 \\
\hline $\mathrm{H}(16 \mathrm{~A})$ & 871 & 1678 & 2435 & 90 \\
\hline $\mathrm{H}(16 \mathrm{~B})$ & 1123 & 642 & 2095 & 90 \\
\hline $\mathrm{H}(16 \mathrm{C})$ & -382 & 1048 & 2089 & 90 \\
\hline $\mathrm{H}(17 \mathrm{~A})$ & 1247 & 3342 & 2449 & 78 \\
\hline $\mathrm{H}(17 \mathrm{~B})$ & 301 & 4234 & 2118 & 78 \\
\hline $\mathrm{H}(17 \mathrm{C})$ & 1882 & 4224 & 2108 & 78 \\
\hline $\mathrm{H}(18 \mathrm{~A})$ & 598 & 5086 & 1168 & 71 \\
\hline $\mathrm{H}(18 \mathrm{~B})$ & -675 & 4726 & 717 & 71 \\
\hline $\mathrm{H}(18 \mathrm{C})$ & 789 & 4781 & 555 & 71 \\
\hline $\mathrm{H}(19 \mathrm{~A})$ & -434 & 1891 & -97 & 71 \\
\hline $\mathrm{H}(19 \mathrm{~B})$ & 379 & 2966 & -132 & 71 \\
\hline $\mathrm{H}(19 \mathrm{C})$ & -1142 & 3003 & -30 & 71 \\
\hline $\mathrm{H}(20 \mathrm{~A})$ & -523 & 534 & 402 & 89 \\
\hline $\mathrm{H}(20 \mathrm{~B})$ & -1204 & 427 & 938 & 89 \\
\hline $\mathrm{H}(20 \mathrm{C})$ & 284 & -20 & 949 & 89 \\
\hline
\end{tabular}




\begin{tabular}{lrrrr}
$\mathrm{H}(21 \mathrm{~A})$ & 1940 & 854 & 130 & 40 \\
$\mathrm{H}(21 \mathrm{~B})$ & 3357 & 1315 & 1 & 40 \\
$\mathrm{H}(23)$ & 2014 & -588 & 857 & 47 \\
$\mathrm{H}(24)$ & 3242 & -1827 & 1458 & 59 \\
$\mathrm{H}(25)$ & 5583 & -1808 & 1653 & 60 \\
$\mathrm{H}(26)$ & 6716 & -591 & 1180 & 54 \\
$\mathrm{H}(27)$ & 5581 & 657 & 579 & 48 \\
$\mathrm{H}(28 \mathrm{~A})$ & 3330 & 855 & 2112 & 37 \\
$\mathrm{H}(28 \mathrm{~B})$ & 4702 & 754 & 1897 & 37 \\
$\mathrm{H}(30)$ & 6673 & 935 & 2528 & 52 \\
$\mathrm{H}(31)$ & 7892 & 1475 & 3389 & 63 \\
$\mathrm{H}(32)$ & 6832 & 2507 & 3993 & 65 \\
$\mathrm{H}(33)$ & 4508 & 2902 & 3731 & 63 \\
$\mathrm{H}(34)$ & 3312 & 2388 & 2864 & 48 \\
& & & & \\
\hline
\end{tabular}


Table S6. Crystal data and structure refinement for $\left(\mathrm{C}_{5} \mathrm{Me}_{5}\right)_{2} \mathrm{Th}\left(\mathrm{CH}_{3}\right)_{2}(\mathbf{9 c})$.

Identification code

Empirical formula

Formula weight

Temperature

Wavelength

Crystal system

Space group

Unit cell dimensions

Volume

$\mathrm{Z}$

Density (calculated)

Absorption coefficient

$\mathrm{F}(000)$

Crystal size

Theta range for data collection

Index ranges

Reflections collected

Independent reflections

Max. and min. transmission

Refinement method

Data / restraints / parameters

Goodness-of-fit on $\mathrm{F}^{2}$

Final R indices [I $>2 \operatorname{sigma}(\mathrm{I})]$

$\mathrm{R}$ indices (all data)

Largest diff. peak and hole $\operatorname{ccd} 792$

$\mathrm{C}_{22} \mathrm{H}_{36} \mathrm{Th}$

532.55

203(2) K

$0.71073 \AA$

monoclinic

$\mathrm{C} 2 / \mathrm{c}$

$\mathrm{a}=8.3232(14) \AA$

$\mathrm{b}=17.302(3) \AA$

$\beta=117.185(3)^{\circ}$.

$\mathrm{c}=8.4939(15) \AA$

1088.1(3) $\AA^{3}$

2

$1.625 \mathrm{Mg} / \mathrm{m}^{3}$

$6.852 \mathrm{~mm}^{-1}$

516

$0.24 \times 0.10 \times 0.08 \mathrm{~mm}^{3}$

2.70 to $28.10^{\circ}$.

$-9<=\mathrm{h}<=10,-22<=\mathrm{k}<=22,-11<=1<=11$

7884

$2497[\mathrm{R}(\mathrm{int})=0.0226]$

0.6102 and 0.2901

Full-matrix least-squares on $\mathrm{F}^{2}$

2497 / 0 / 151

1.576

$\mathrm{R} 1=0.0326, \mathrm{wR} 2=0.0930$

$\mathrm{R} 1=0.0349, \mathrm{wR} 2=0.0941$

1.512 and -1.878 e. $\AA^{-3}$ 
Table S7. Atomic coordinates ( $\mathrm{x} 10^{4}$ ) and equivalent isotropic displacement parameters $\left(\AA^{2} \times 10^{3}\right)$ for $\left(\mathrm{C}_{5} \mathrm{Me}_{5}\right)_{2} \mathrm{Th}\left(\mathrm{CH}_{3}\right)_{2}(\mathbf{9 c})$. U(eq) is defined as one third of the trace of the orthogonalized $\mathrm{U}^{\mathrm{ij}}$ tensor.

\begin{tabular}{|c|c|c|c|c|}
\hline & $\mathrm{x}$ & $\mathrm{y}$ & z & $\mathrm{U}(\mathrm{eq})$ \\
\hline $\operatorname{Th}(1)$ & $3127(1)$ & 7500 & $3837(1)$ & $32(1)$ \\
\hline$C(1)$ & $2312(14)$ & 7500 & $6293(12)$ & $54(2)$ \\
\hline$C(2)$ & $40(13)$ & 7500 & $1329(14)$ & $62(2)$ \\
\hline$C(3)$ & $3148(8)$ & 6064(4) & $2337(7)$ & $40(4)$ \\
\hline$C(4)$ & 2996(8) & 5899(4) & 3901(9) & $40(3)$ \\
\hline$C(5)$ & 4659(9) & 6096(4) & $5375(7)$ & $40(4)$ \\
\hline$C(6)$ & $5839(7)$ & 6383(4) & $4721(7)$ & $38(2)$ \\
\hline$C(7)$ & 4906(8) & $6362(4)$ & $2844(7)$ & $38(3)$ \\
\hline$C(8)$ & $1697(11)$ & $5942(7)$ & $464(8)$ & $76(5)$ \\
\hline $\mathrm{C}(9)$ & $1355(11)$ & $5572(6)$ & 3983(14) & $77(5)$ \\
\hline$C(10)$ & $5096(14)$ & 6016(6) & $7298(7)$ & $77(5)$ \\
\hline $\mathrm{C}(11)$ & $7751(7)$ & $6660(6)$ & $5828(11)$ & $69(4)$ \\
\hline$C(12)$ & $5650(13)$ & 6614(6) & $1604(10)$ & $65(4)$ \\
\hline$C\left(3^{\prime}\right)$ & $2465(8)$ & $5846(4)$ & $3124(8)$ & $42(3)$ \\
\hline$C\left(4^{\prime}\right)$ & $3578(10)$ & 5886(4) & 4981(8) & $47(3)$ \\
\hline$C\left(5^{\prime}\right)$ & $5306(9)$ & 6156(4) & $5281(8)$ & $54(5)$ \\
\hline$C\left(6^{\prime}\right)$ & 5261(9) & $6283(5)$ & $3610(11)$ & $46(4)$ \\
\hline$C\left(7^{\prime}\right)$ & $3505(10)$ & 6091(4) & $2276(7)$ & $42(4)$ \\
\hline$C\left(8^{\prime}\right)$ & $518(9)$ & $5588(6)$ & $2211(14)$ & $79(5)$ \\
\hline $\mathrm{C}\left(9^{\prime}\right)$ & $3023(17)$ & $5678(6)$ & $6389(12)$ & $81(5)$ \\
\hline$C\left(10^{\prime}\right)$ & $6910(12)$ & $6285(7)$ & $7065(11)$ & $139(13)$ \\
\hline$C\left(11^{\prime}\right)$ & $6807(13)$ & $6571(7)$ & $3304(18)$ & $190(20)$ \\
\hline$C\left(12^{\prime}\right)$ & $2857(17)$ & $6140(7)$ & $304(8)$ & $87(6)$ \\
\hline
\end{tabular}


Table S8. Bond lengths $[\AA]$ and angles $\left[^{\circ}\right]$ for $\left(\mathrm{C}_{5} \mathrm{Me}_{5}\right)_{2} \mathrm{Th}\left(\mathrm{CH}_{3}\right)_{2}(\mathbf{9 c})$.

\begin{tabular}{|c|c|}
\hline $\operatorname{Th}(1)-C(1)$ & $2.471(8)$ \\
\hline $\operatorname{Th}(1)-C(2)$ & $2.478(9)$ \\
\hline $\operatorname{Th}(1)-\mathrm{C}(4) \# 1$ & $2.773(7)$ \\
\hline $\operatorname{Th}(1)-C(4)$ & $2.773(7)$ \\
\hline $\operatorname{Th}(1)-\mathrm{C}(5) \# 1$ & $2.776(11)$ \\
\hline $\operatorname{Th}(1)-C(5)$ & $2.776(6)$ \\
\hline $\operatorname{Th}(1)-\mathrm{C}(3)$ & $2.797(7)$ \\
\hline $\operatorname{Th}(1)-\mathrm{C}(3) \# 1$ & $2.797(7)$ \\
\hline $\operatorname{Th}(1)-\mathrm{C}(6) \# 1$ & $2.802(13)$ \\
\hline $\operatorname{Th}(1)-C(6)$ & $2.802(5)$ \\
\hline $\operatorname{Th}(1)-C(7) \# 1$ & $2.814(14)$ \\
\hline $\operatorname{Th}(1)-C(7)$ & $2.814(6)$ \\
\hline $\mathrm{C}(3)-\mathrm{C}\left(3^{\prime}\right)$ & $1.122(9)$ \\
\hline$C(3)-C(4)$ & 1.4200 \\
\hline$C(3)-C(7)$ & 1.4200 \\
\hline$C(3)-C(8)$ & 1.5095 \\
\hline$C(3)-C\left(6^{\prime}\right)$ & $1.633(9)$ \\
\hline $\mathrm{C}(3)-\mathrm{C}\left(12^{\prime}\right)$ & $1.635(7)$ \\
\hline $\mathrm{C}(4)-\mathrm{C}\left(3^{\prime}\right)$ & $0.611(8)$ \\
\hline$C(4)-C\left(4^{\prime}\right)$ & $0.819(8)$ \\
\hline$C(4)-C(5)$ & 1.4200 \\
\hline$C(4)-C(9)$ & 1.5095 \\
\hline$C(4)-C\left(7^{\prime}\right)$ & $1.652(8)$ \\
\hline$C(4)-C\left(5^{\prime}\right)$ & $1.791(8)$ \\
\hline$C(4)-C\left(8^{\prime}\right)$ & $1.975(8)$ \\
\hline$C(5)-C\left(5^{\prime}\right)$ & $0.588(8)$ \\
\hline$C(5)-C\left(4^{\prime}\right)$ & $0.883(8)$ \\
\hline$C(5)-C(6)$ & 1.4200 \\
\hline$C(5)-C(10)$ & 1.5095 \\
\hline $\mathrm{C}(5)-\mathrm{C}\left(10^{\prime}\right)$ & $1.794(8)$ \\
\hline$C(5)-C\left(6^{\prime}\right)$ & $1.813(8)$ \\
\hline $\mathrm{C}(5)-\mathrm{C}\left(3^{\prime}\right)$ & $1.997(7)$ \\
\hline $\mathrm{C}(6)-\mathrm{C}\left(6^{\prime}\right)$ & $0.859(9)$ \\
\hline$C(6)-C\left(5^{\prime}\right)$ & $0.877(9)$ \\
\hline
\end{tabular}




\begin{tabular}{|c|c|}
\hline$C(6)-C(7)$ & 1.4200 \\
\hline $\mathrm{C}(6)-\mathrm{C}(11)$ & 1.5095 \\
\hline $\mathrm{C}(6)-\mathrm{C}\left(11^{\prime}\right)$ & $1.758(9)$ \\
\hline $\mathrm{C}(6)-\mathrm{C}\left(10^{\prime}\right)$ & $1.778(9)$ \\
\hline $\mathrm{C}(7)-\mathrm{C}\left(6^{\prime}\right)$ & $0.595(9)$ \\
\hline$C(7)-C\left(7^{\prime}\right)$ & $1.140(10)$ \\
\hline C(7)-C(11') & $1.491(9)$ \\
\hline$C(7)-C(12)$ & 1.5095 \\
\hline$C(7)-C\left(5^{\prime}\right)$ & $1.974(8)$ \\
\hline $\mathrm{C}(8)-\mathrm{C}\left(12^{\prime}\right)$ & $1.089(14)$ \\
\hline $\mathrm{C}(8)-\mathrm{C}\left(7^{\prime}\right)$ & $1.608(8)$ \\
\hline $\mathrm{C}(9)-\mathrm{C}\left(8^{\prime}\right)$ & $1.339(12)$ \\
\hline $\mathrm{C}(9)-\mathrm{C}\left(3^{\prime}\right)$ & $1.492(9)$ \\
\hline C(9)-C(4') & $1.734(8)$ \\
\hline $\mathrm{C}(9)-\mathrm{C}\left(9^{\prime}\right)$ & $1.883(11)$ \\
\hline $\mathrm{C}(10)-\mathrm{C}\left(9^{\prime}\right)$ & $1.643(12)$ \\
\hline $\mathrm{C}(10)-\mathrm{C}\left(10^{\prime}\right)$ & $1.676(12)$ \\
\hline$C(10)-C\left(4^{\prime}\right)$ & $1.803(8)$ \\
\hline$C(10)-C\left(5^{\prime}\right)$ & $1.816(8)$ \\
\hline $\mathrm{C}(11)-\mathrm{C}\left(10^{\prime}\right)$ & $1.638(13)$ \\
\hline$C(11)-C\left(11^{\prime}\right)$ & $1.923(14)$ \\
\hline$C(12)-C\left(11^{\prime}\right)$ & $1.322(14)$ \\
\hline $\mathrm{C}(12)-\mathrm{C}\left(6^{\prime}\right)$ & $1.960(8)$ \\
\hline $\mathrm{C}\left(3^{\prime}\right)-\mathrm{C}\left(4^{\prime}\right)$ & 1.4200 \\
\hline $\mathrm{C}\left(3^{\prime}\right)-\mathrm{C}\left(7^{\prime}\right)$ & 1.4200 \\
\hline $\mathrm{C}\left(3^{\prime}\right)-\mathrm{C}\left(8^{\prime}\right)$ & 1.5095 \\
\hline$C\left(4^{\prime}\right)-C\left(5^{\prime}\right)$ & 1.4200 \\
\hline $\mathrm{C}\left(4^{\prime}\right)-\mathrm{C}\left(9^{\prime}\right)$ & 1.5095 \\
\hline$C\left(5^{\prime}\right)-C\left(6^{\prime}\right)$ & 1.4200 \\
\hline $\mathrm{C}\left(5^{\prime}\right)-\mathrm{C}\left(10^{\prime}\right)$ & 1.5095 \\
\hline$C\left(6^{\prime}\right)-C\left(7^{\prime}\right)$ & 1.4200 \\
\hline $\mathrm{C}\left(6^{\prime}\right)-\mathrm{C}\left(11^{\prime}\right)$ & 1.5095 \\
\hline$C\left(7^{\prime}\right)-C\left(12^{\prime}\right)$ & 1.5095 \\
\hline $\mathrm{C}(1)-\mathrm{Th}(1)-\mathrm{C}(2)$ & $98.6(4)$ \\
\hline $\mathrm{C}(1)-\mathrm{Th}(1)-\mathrm{C}(4) \# 1$ & $87.4(4)$ \\
\hline
\end{tabular}




\begin{tabular}{|c|c|}
\hline $\mathrm{C}(2)-\mathrm{Th}(1)-\mathrm{C}(4) \# 1$ & $89.0(4)$ \\
\hline $\mathrm{C}(1)-\mathrm{Th}(1)-\mathrm{C}(4)$ & $87.43(12)$ \\
\hline $\mathrm{C}(2)-\mathrm{Th}(1)-\mathrm{C}(4)$ & $88.98(14)$ \\
\hline $\mathrm{C}(4) \# 1-\mathrm{Th}(1)-\mathrm{C}(4)$ & $174.1(4)$ \\
\hline $\mathrm{C}(1)-\mathrm{Th}(1)-\mathrm{C}(5) \# 1$ & $81.2(4)$ \\
\hline $\mathrm{C}(2)-\mathrm{Th}(1)-\mathrm{C}(5) \# 1$ & $118.5(3)$ \\
\hline $\mathrm{C}(4) \# 1-\mathrm{Th}(1)-\mathrm{C}(5) \# 1$ & $29.65(11)$ \\
\hline $\mathrm{C}(4)-\mathrm{Th}(1)-\mathrm{C}(5) \# 1$ & $151.4(3)$ \\
\hline $\mathrm{C}(1)-\mathrm{Th}(1)-\mathrm{C}(5)$ & $81.21(15)$ \\
\hline $\mathrm{C}(2)-\operatorname{Th}(1)-\mathrm{C}(5)$ & $118.52(14)$ \\
\hline $\mathrm{C}(4) \# 1-\mathrm{Th}(1)-\mathrm{C}(5)$ & $151.4(4)$ \\
\hline $\mathrm{C}(4)-\operatorname{Th}(1)-\mathrm{C}(5)$ & $29.65(6)$ \\
\hline $\mathrm{C}(5) \# 1-\mathrm{Th}(1)-\mathrm{C}(5)$ & $122.0(3)$ \\
\hline $\mathrm{C}(1)-\mathrm{Th}(1)-\mathrm{C}(3)$ & $116.50(13)$ \\
\hline $\mathrm{C}(2)-\mathrm{Th}(1)-\mathrm{C}(3)$ & $80.11(17)$ \\
\hline $\mathrm{C}(4) \# 1-\mathrm{Th}(1)-\mathrm{C}(3)$ & $154.8(4)$ \\
\hline $\mathrm{C}(4)-\operatorname{Th}(1)-\mathrm{C}(3)$ & $29.53(7)$ \\
\hline $\mathrm{C}(5) \# 1-\mathrm{Th}(1)-\mathrm{C}(3)$ & $153.4(4)$ \\
\hline $\mathrm{C}(5)-\mathrm{Th}(1)-\mathrm{C}(3)$ & $48.69(9)$ \\
\hline $\mathrm{C}(1)-\mathrm{Th}(1)-\mathrm{C}(3) \# 1$ & $116.50(13)$ \\
\hline $\mathrm{C}(2)-\mathrm{Th}(1)-\mathrm{C}(3) \# 1$ & $80.11(17)$ \\
\hline $\mathrm{C}(4) \# 1-\mathrm{Th}(1)-\mathrm{C}(3) \# 1$ & $29.5(4)$ \\
\hline $\mathrm{C}(4)-\mathrm{Th}(1)-\mathrm{C}(3) \# 1$ & $154.8(2)$ \\
\hline $\mathrm{C}(5) \# 1-\mathrm{Th}(1)-\mathrm{C}(3) \# 1$ & $48.7(3)$ \\
\hline $\mathrm{C}(5)-\mathrm{Th}(1)-\mathrm{C}(3) \# 1$ & $153.4(2)$ \\
\hline $\mathrm{C}(3)-\mathrm{Th}(1)-\mathrm{C}(3) \# 1$ & $125.4(3)$ \\
\hline $\mathrm{C}(1)-\mathrm{Th}(1)-\mathrm{C}(6) \# 1$ & $105.8(4)$ \\
\hline $\mathrm{C}(2)-\mathrm{Th}(1)-\mathrm{C}(6) \# 1$ & $128.5(3)$ \\
\hline $\mathrm{C}(4) \# 1-\mathrm{Th}(1)-\mathrm{C}(6) \# 1$ & $48.7(2)$ \\
\hline $\mathrm{C}(4)-\mathrm{Th}(1)-\mathrm{C}(6) \# 1$ & $135.9(2)$ \\
\hline $\mathrm{C}(5) \# 1-\mathrm{Th}(1)-\mathrm{C}(6) \# 1$ & $29.49(16)$ \\
\hline $\mathrm{C}(5)-\mathrm{Th}(1)-\mathrm{C}(6) \# 1$ & 109.7(3) \\
\hline $\mathrm{C}(3)-\mathrm{Th}(1)-\mathrm{C}(6) \# 1$ & $124.3(3)$ \\
\hline $\mathrm{C}(3) \# 1-\mathrm{Th}(1)-\mathrm{C}(6) \# 1$ & $48.5(3)$ \\
\hline $\mathrm{C}(1)-\mathrm{Th}(1)-\mathrm{C}(6)$ & $105.8(2)$ \\
\hline $\mathrm{C}(2)-\mathrm{Th}(1)-\mathrm{C}(6)$ & $128.51(17)$ \\
\hline
\end{tabular}




\begin{tabular}{|c|c|}
\hline $\mathrm{C}(4) \# 1-\mathrm{Th}(1)-\mathrm{C}(6)$ & $135.9(4)$ \\
\hline $\mathrm{C}(4)-\mathrm{Th}(1)-\mathrm{C}(6)$ & $48.67(9)$ \\
\hline $\mathrm{C}(5) \# 1-\mathrm{Th}(1)-\mathrm{C}(6)$ & 109.7(3) \\
\hline $\mathrm{C}(5)-\mathrm{Th}(1)-\mathrm{C}(6)$ & $29.49(6)$ \\
\hline $\mathrm{C}(3)-\mathrm{Th}(1)-\mathrm{C}(6)$ & $48.46(9)$ \\
\hline $\mathrm{C}(3) \# 1-\mathrm{Th}(1)-\mathrm{C}(6)$ & $124.3(2)$ \\
\hline $\mathrm{C}(6) \# 1-\mathrm{Th}(1)-\mathrm{C}(6)$ & $87.3(2)$ \\
\hline $\mathrm{C}(1)-\mathrm{Th}(1)-\mathrm{C}(7) \# 1$ & $129.7(2)$ \\
\hline $\mathrm{C}(2)-\mathrm{Th}(1)-\mathrm{C}(7) \# 1$ & $102.9(4)$ \\
\hline $\mathrm{C}(4) \# 1-\mathrm{Th}(1)-\mathrm{C}(7) \# 1$ & $48.6(2)$ \\
\hline $\mathrm{C}(4)-\mathrm{Th}(1)-\mathrm{C}(7) \# 1$ & $137.3(2)$ \\
\hline $\mathrm{C}(5) \# 1-\mathrm{Th}(1)-\mathrm{C}(7) \# 1$ & $48.53(19)$ \\
\hline $\mathrm{C}(5)-\mathrm{Th}(1)-\mathrm{C}(7) \# 1$ & 124.1(3) \\
\hline $\mathrm{C}(3)-\mathrm{Th}(1)-\mathrm{C}(7) \# 1$ & $111.8(3)$ \\
\hline $\mathrm{C}(3) \# 1-\mathrm{Th}(1)-\mathrm{C}(7) \# 1$ & $29.3(2)$ \\
\hline $\mathrm{C}(6) \# 1-\mathrm{Th}(1)-\mathrm{C}(7) \# 1$ & $29.29(13)$ \\
\hline $\mathrm{C}(6)-\mathrm{Th}(1)-\mathrm{C}(7) \# 1$ & $95.3(3)$ \\
\hline $\mathrm{C}(1)-\mathrm{Th}(1)-\mathrm{C}(7)$ & $129.65(15)$ \\
\hline $\mathrm{C}(2)-\operatorname{Th}(1)-\mathrm{C}(7)$ & $102.9(2)$ \\
\hline $\mathrm{C}(4) \# 1-\mathrm{Th}(1)-\mathrm{C}(7)$ & $137.3(4)$ \\
\hline $\mathrm{C}(4)-\mathrm{Th}(1)-\mathrm{C}(7)$ & $48.55(9)$ \\
\hline $\mathrm{C}(5) \# 1-\mathrm{Th}(1)-\mathrm{C}(7)$ & $124.1(4)$ \\
\hline $\mathrm{C}(5)-\operatorname{Th}(1)-\mathrm{C}(7)$ & $48.53(8)$ \\
\hline $\mathrm{C}(3)-\mathrm{Th}(1)-\mathrm{C}(7)$ & $29.32(6)$ \\
\hline $\mathrm{C}(3) \# 1-\mathrm{Th}(1)-\mathrm{C}(7)$ & $111.8(2)$ \\
\hline $\mathrm{C}(6) \# 1-\mathrm{Th}(1)-\mathrm{C}(7)$ & $95.3(3)$ \\
\hline $\mathrm{C}(6)-\mathrm{Th}(1)-\mathrm{C}(7)$ & $29.29(6)$ \\
\hline $\mathrm{C}(7) \# 1-\mathrm{Th}(1)-\mathrm{C}(7)$ & $88.8(2)$ \\
\hline $\mathrm{C}\left(3^{\prime}\right)-\mathrm{C}(3)-\mathrm{C}(4)$ & $24.4(4)$ \\
\hline $\mathrm{C}\left(3^{\prime}\right)-\mathrm{C}(3)-\mathrm{C}(7)$ & $132.3(4)$ \\
\hline $\mathrm{C}(4)-\mathrm{C}(3)-\mathrm{C}(7)$ & 108.0 \\
\hline $\mathrm{C}\left(3^{\prime}\right)-\mathrm{C}(3)-\mathrm{C}(8)$ & $101.6(4)$ \\
\hline $\mathrm{C}(4)-\mathrm{C}(3)-\mathrm{C}(8)$ & 126.0 \\
\hline $\mathrm{C}(7)-\mathrm{C}(3)-\mathrm{C}(8)$ & 126.0 \\
\hline $\mathrm{C}\left(3^{\prime}\right)-\mathrm{C}(3)-\mathrm{C}\left(6^{\prime}\right)$ & $111.6(5)$ \\
\hline $\mathrm{C}(4)-\mathrm{C}(3)-\mathrm{C}\left(6^{\prime}\right)$ & $87.5(3)$ \\
\hline
\end{tabular}




\begin{tabular}{|c|c|}
\hline $\mathrm{C}(7)-\mathrm{C}(3)-\mathrm{C}\left(6^{\prime}\right)$ & $21.0(3)$ \\
\hline $\mathrm{C}(8)-\mathrm{C}(3)-\mathrm{C}\left(6^{\prime}\right)$ & $146.2(3)$ \\
\hline $\mathrm{C}\left(3^{\prime}\right)-\mathrm{C}(3)-\mathrm{C}\left(12^{\prime}\right)$ & $141.7(7)$ \\
\hline $\mathrm{C}(4)-\mathrm{C}(3)-\mathrm{C}\left(12^{\prime}\right)$ & $166.0(5)$ \\
\hline $\mathrm{C}(7)-\mathrm{C}(3)-\mathrm{C}\left(12^{\prime}\right)$ & $85.8(5)$ \\
\hline $\mathrm{C}(8)-\mathrm{C}(3)-\mathrm{C}\left(12^{\prime}\right)$ & $40.3(5)$ \\
\hline $\mathrm{C}\left(6^{\prime}\right)-\mathrm{C}(3)-\mathrm{C}\left(12^{\prime}\right)$ & $106.0(5)$ \\
\hline $\mathrm{C}\left(3^{\prime}\right)-\mathrm{C}(3)-\mathrm{Th}(1)$ & $85.2(5)$ \\
\hline $\mathrm{C}(4)-\mathrm{C}(3)-\mathrm{Th}(1)$ & $74.3(2)$ \\
\hline$C(7)-C(3)-T h(1)$ & 76.01(19) \\
\hline $\mathrm{C}(8)-\mathrm{C}(3)-\mathrm{Th}(1)$ & $115.82(18)$ \\
\hline $\mathrm{C}\left(6^{\prime}\right)-\mathrm{C}(3)-\mathrm{Th}(1)$ & $73.8(4)$ \\
\hline $\mathrm{C}\left(12^{\prime}\right)-\mathrm{C}(3)-\mathrm{Th}(1)$ & $112.4(6)$ \\
\hline $\mathrm{C}\left(3^{\prime}\right)-\mathrm{C}(4)-\mathrm{C}\left(4^{\prime}\right)$ & $166.5(14)$ \\
\hline $\mathrm{C}\left(3^{\prime}\right)-\mathrm{C}(4)-\mathrm{C}(5)$ & $157.2(8)$ \\
\hline $\mathrm{C}\left(4^{\prime}\right)-\mathrm{C}(4)-\mathrm{C}(5)$ & $34.9(6)$ \\
\hline$C\left(3^{\prime}\right)-C(4)-C(3)$ & $49.4(8)$ \\
\hline $\mathrm{C}\left(4^{\prime}\right)-\mathrm{C}(4)-\mathrm{C}(3)$ & $142.5(6)$ \\
\hline$C(5)-C(4)-C(3)$ & 108.0 \\
\hline C(3')-C(4)-C(9) & $76.7(8)$ \\
\hline $\mathrm{C}\left(4^{\prime}\right)-\mathrm{C}(4)-\mathrm{C}(9)$ & $91.3(6)$ \\
\hline $\mathrm{C}(5)-\mathrm{C}(4)-\mathrm{C}(9)$ & 126.0 \\
\hline $\mathrm{C}(3)-\mathrm{C}(4)-\mathrm{C}(9)$ & 126.0 \\
\hline $\mathrm{C}\left(3^{\prime}\right)-\mathrm{C}(4)-\mathrm{C}\left(7^{\prime}\right)$ & $57.5(6)$ \\
\hline $\mathrm{C}\left(4^{\prime}\right)-\mathrm{C}(4)-\mathrm{C}\left(7^{\prime}\right)$ & $134.0(7)$ \\
\hline $\mathrm{C}(5)-\mathrm{C}(4)-\mathrm{C}\left(7^{\prime}\right)$ & $99.8(3)$ \\
\hline $\mathrm{C}(3)-\mathrm{C}(4)-\mathrm{C}\left(7^{\prime}\right)$ & $8.6(3)$ \\
\hline $\mathrm{C}(9)-\mathrm{C}(4)-\mathrm{C}\left(7^{\prime}\right)$ & $134.2(3)$ \\
\hline $\mathrm{C}\left(3^{\prime}\right)-\mathrm{C}(4)-\mathrm{C}\left(5^{\prime}\right)$ & $140.8(9)$ \\
\hline $\mathrm{C}\left(4^{\prime}\right)-\mathrm{C}(4)-\mathrm{C}\left(5^{\prime}\right)$ & $50.6(4)$ \\
\hline $\mathrm{C}(5)-\mathrm{C}(4)-\mathrm{C}\left(5^{\prime}\right)$ & $16.4(3)$ \\
\hline $\mathrm{C}(3)-\mathrm{C}(4)-\mathrm{C}\left(5^{\prime}\right)$ & $92.0(3)$ \\
\hline $\mathrm{C}(9)-\mathrm{C}(4)-\mathrm{C}\left(5^{\prime}\right)$ & $141.8(3)$ \\
\hline$C\left(7^{\prime}\right)-C(4)-C\left(5^{\prime}\right)$ & $83.6(3)$ \\
\hline $\mathrm{C}\left(3^{\prime}\right)-\mathrm{C}(4)-\mathrm{C}\left(8^{\prime}\right)$ & $34.2(5)$ \\
\hline$C\left(4^{\prime}\right)-C(4)-C\left(8^{\prime}\right)$ & $133.9(7)$ \\
\hline
\end{tabular}




\begin{tabular}{|c|c|}
\hline $\mathrm{C}(5)-\mathrm{C}(4)-\mathrm{C}\left(8^{\prime}\right)$ & $168.6(4)$ \\
\hline $\mathrm{C}(3)-\mathrm{C}(4)-\mathrm{C}\left(8^{\prime}\right)$ & $83.4(4)$ \\
\hline $\mathrm{C}(9)-\mathrm{C}(4)-\mathrm{C}\left(8^{\prime}\right)$ & $42.7(4)$ \\
\hline $\mathrm{C}\left(7^{\prime}\right)-\mathrm{C}(4)-\mathrm{C}\left(8^{\prime}\right)$ & $91.6(3)$ \\
\hline $\mathrm{C}\left(5^{\prime}\right)-\mathrm{C}(4)-\mathrm{C}\left(8^{\prime}\right)$ & $174.7(5)$ \\
\hline $\mathrm{C}\left(3^{\prime}\right)-\mathrm{C}(4)-\mathrm{Th}(1)$ & $98.3(10)$ \\
\hline $\mathrm{C}\left(4^{\prime}\right)-\mathrm{C}(4)-\mathrm{Th}(1)$ & $92.4(7)$ \\
\hline $\mathrm{C}(5)-\mathrm{C}(4)-\mathrm{Th}(1)$ & $75.29(19)$ \\
\hline $\mathrm{C}(3)-\mathrm{C}(4)-\operatorname{Th}(1)$ & $76.1(2)$ \\
\hline C(9)-C(4)-Th(1) & $114.82(18)$ \\
\hline $\mathrm{C}\left(7^{\prime}\right)-\mathrm{C}(4)-\mathrm{Th}(1)$ & $75.8(4)$ \\
\hline $\mathrm{C}\left(5^{\prime}\right)-\mathrm{C}(4)-\mathrm{Th}(1)$ & $74.0(3)$ \\
\hline $\mathrm{C}\left(8^{\prime}\right)-\mathrm{C}(4)-\mathrm{Th}(1)$ & $107.2(5)$ \\
\hline$C\left(5^{\prime}\right)-C(5)-C\left(4^{\prime}\right)$ & $149.2(12)$ \\
\hline $\mathrm{C}\left(5^{\prime}\right)-\mathrm{C}(5)-\mathrm{C}(4)$ & $120.5(7)$ \\
\hline $\mathrm{C}\left(4^{\prime}\right)-\mathrm{C}(5)-\mathrm{C}(4)$ & $32.0(5)$ \\
\hline $\mathrm{C}\left(5^{\prime}\right)-\mathrm{C}(5)-\mathrm{C}(6)$ & $17.6(8)$ \\
\hline$C\left(4^{\prime}\right)-C(5)-C(6)$ & $139.7(5)$ \\
\hline$C(4)-C(5)-C(6)$ & 108.0 \\
\hline $\mathrm{C}\left(5^{\prime}\right)-\mathrm{C}(5)-\mathrm{C}(10)$ & 112.3(7) \\
\hline $\mathrm{C}\left(4^{\prime}\right)-\mathrm{C}(5)-\mathrm{C}(10)$ & $94.1(5)$ \\
\hline $\mathrm{C}(4)-\mathrm{C}(5)-\mathrm{C}(10)$ & 126.0 \\
\hline $\mathrm{C}(6)-\mathrm{C}(5)-\mathrm{C}(10)$ & 126.0 \\
\hline $\mathrm{C}\left(5^{\prime}\right)-\mathrm{C}(5)-\mathrm{C}\left(10^{\prime}\right)$ & $52.4(6)$ \\
\hline$C\left(4^{\prime}\right)-C(5)-C\left(10^{\prime}\right)$ & $152.8(7)$ \\
\hline $\mathrm{C}(4)-\mathrm{C}(5)-\mathrm{C}\left(10^{\prime}\right)$ & $171.9(5)$ \\
\hline $\mathrm{C}(6)-\mathrm{C}(5)-\mathrm{C}\left(10^{\prime}\right)$ & $66.0(4)$ \\
\hline $\mathrm{C}(10)-\mathrm{C}(5)-\mathrm{C}\left(10^{\prime}\right)$ & $60.3(4)$ \\
\hline $\mathrm{C}\left(5^{\prime}\right)-\mathrm{C}(5)-\mathrm{C}\left(6^{\prime}\right)$ & $40.7(5)$ \\
\hline $\mathrm{C}\left(4^{\prime}\right)-\mathrm{C}(5)-\mathrm{C}\left(6^{\prime}\right)$ & $112.2(6)$ \\
\hline $\mathrm{C}(4)-\mathrm{C}(5)-\mathrm{C}\left(6^{\prime}\right)$ & $80.8(3)$ \\
\hline $\mathrm{C}(6)-\mathrm{C}(5)-\mathrm{C}\left(6^{\prime}\right)$ & $27.5(3)$ \\
\hline$C(10)-C(5)-C\left(6^{\prime}\right)$ & $152.9(3)$ \\
\hline $\mathrm{C}\left(10^{\prime}\right)-\mathrm{C}(5)-\mathrm{C}\left(6^{\prime}\right)$ & $92.7(4)$ \\
\hline $\mathrm{C}\left(5^{\prime}\right)-\mathrm{C}(5)-\mathrm{C}\left(3^{\prime}\right)$ & $113.7(8)$ \\
\hline$C\left(4^{\prime}\right)-C(5)-C\left(3^{\prime}\right)$ & $38.7(3)$ \\
\hline
\end{tabular}




\begin{tabular}{|c|c|}
\hline $\mathrm{C}(4)-\mathrm{C}(5)-\mathrm{C}\left(3^{\prime}\right)$ & $6.8(2)$ \\
\hline $\mathrm{C}(6)-\mathrm{C}(5)-\mathrm{C}\left(3^{\prime}\right)$ & 101.2(3) \\
\hline $\mathrm{C}(10)-\mathrm{C}(5)-\mathrm{C}\left(3^{\prime}\right)$ & $132.8(3)$ \\
\hline $\mathrm{C}\left(10^{\prime}\right)-\mathrm{C}(5)-\mathrm{C}\left(3^{\prime}\right)$ & $165.8(5)$ \\
\hline $\mathrm{C}\left(6^{\prime}\right)-\mathrm{C}(5)-\mathrm{C}\left(3^{\prime}\right)$ & $74.0(3)$ \\
\hline $\mathrm{C}\left(5^{\prime}\right)-\mathrm{C}(5)-\mathrm{Th}(1)$ & $92.0(10)$ \\
\hline $\mathrm{C}\left(4^{\prime}\right)-\mathrm{C}(5)-\mathrm{Th}(1)$ & $90.7(6)$ \\
\hline$C(4)-C(5)-T h(1)$ & $75.1(2)$ \\
\hline $\mathrm{C}(6)-\mathrm{C}(5)-\operatorname{Th}(1)$ & $76.23(19)$ \\
\hline $\mathrm{C}(10)-\mathrm{C}(5)-\mathrm{Th}(1)$ & 114.94(19) \\
\hline$C\left(10^{\prime}\right)-C(5)-T h(1)$ & $107.7(5)$ \\
\hline $\mathrm{C}\left(6^{\prime}\right)-\mathrm{C}(5)-\mathrm{Th}(1)$ & $72.3(3)$ \\
\hline $\mathrm{C}\left(3^{\prime}\right)-\mathrm{C}(5)-\mathrm{Th}(1)$ & $73.5(3)$ \\
\hline $\mathrm{C}\left(6^{\prime}\right)-\mathrm{C}(6)-\mathrm{C}\left(5^{\prime}\right)$ & 109.7(9) \\
\hline $\mathrm{C}\left(6^{\prime}\right)-\mathrm{C}(6)-\mathrm{C}(7)$ & $10.3(7)$ \\
\hline $\mathrm{C}\left(5^{\prime}\right)-\mathrm{C}(6)-\mathrm{C}(7)$ & $116.4(5)$ \\
\hline$C\left(6^{\prime}\right)-C(6)-C(5)$ & $102.6(5)$ \\
\hline$C\left(5^{\prime}\right)-C(6)-C(5)$ & $11.7(5)$ \\
\hline$C(7)-C(6)-C(5)$ & 108.0 \\
\hline $\mathrm{C}\left(6^{\prime}\right)-\mathrm{C}(6)-\mathrm{C}(11)$ & $130.6(5)$ \\
\hline $\mathrm{C}\left(5^{\prime}\right)-\mathrm{C}(6)-\mathrm{C}(11)$ & $117.0(5)$ \\
\hline$C(7)-C(6)-C(11)$ & 126.0 \\
\hline$C(5)-C(6)-C(11)$ & 126.0 \\
\hline $\mathrm{C}\left(6^{\prime}\right)-\mathrm{C}(6)-\mathrm{C}\left(11^{\prime}\right)$ & $59.1(5)$ \\
\hline$C\left(5^{\prime}\right)-C(6)-C\left(11^{\prime}\right)$ & $163.2(11)$ \\
\hline$C(7)-C(6)-C\left(11^{\prime}\right)$ & $54.7(4)$ \\
\hline $\mathrm{C}(5)-\mathrm{C}(6)-\mathrm{C}\left(11^{\prime}\right)$ & $161.4(5)$ \\
\hline $\mathrm{C}(11)-\mathrm{C}(6)-\mathrm{C}\left(11^{\prime}\right)$ & $71.7(4)$ \\
\hline$C\left(6^{\prime}\right)-C(6)-C\left(10^{\prime}\right)$ & $162.5(10)$ \\
\hline $\mathrm{C}\left(5^{\prime}\right)-\mathrm{C}(6)-\mathrm{C}\left(10^{\prime}\right)$ & $58.0(5)$ \\
\hline$C(7)-C(6)-C\left(10^{\prime}\right)$ & $172.7(5)$ \\
\hline $\mathrm{C}(5)-\mathrm{C}(6)-\mathrm{C}\left(10^{\prime}\right)$ & $67.2(4)$ \\
\hline$C(11)-C(6)-C\left(10^{\prime}\right)$ & $59.1(4)$ \\
\hline $\mathrm{C}\left(11^{\prime}\right)-\mathrm{C}(6)-\mathrm{C}\left(10^{\prime}\right)$ & $129.2(4)$ \\
\hline $\mathrm{C}\left(6^{\prime}\right)-\mathrm{C}(6)-\mathrm{Th}(1)$ & $82.3(6)$ \\
\hline $\mathrm{C}\left(5^{\prime}\right)-\mathrm{C}(6)-\mathrm{Th}(1)$ & $84.8(6)$ \\
\hline
\end{tabular}




\begin{tabular}{|c|c|}
\hline$C(7)-C(6)-T h(1)$ & $75.8(2)$ \\
\hline$C(5)-C(6)-T h(1)$ & $74.3(2)$ \\
\hline$C(11)-C(6)-T h(1)$ & $116.02(19)$ \\
\hline$C\left(11^{\prime}\right)-C(6)-T h(1)$ & $104.7(5)$ \\
\hline$C\left(10^{\prime}\right)-C(6)-T h(1)$ & $107.2(5)$ \\
\hline$C\left(6^{\prime}\right)-C(7)-C\left(7^{\prime}\right)$ & $105.6(10)$ \\
\hline$C\left(6^{\prime}\right)-C(7)-C(6)$ & $15.0(11)$ \\
\hline$C\left(7^{\prime}\right)-C(7)-C(6)$ & $114.5(4)$ \\
\hline$C\left(6^{\prime}\right)-C(7)-C(3)$ & $100.1(8)$ \\
\hline $\mathrm{C}\left(7^{\prime}\right)-\mathrm{C}(7)-\mathrm{C}(3)$ & $7.6(4)$ \\
\hline$C(6)-C(7)-C(3)$ & 108.0 \\
\hline$C\left(6^{\prime}\right)-C(7)-C\left(11^{\prime}\right)$ & $80.3(9)$ \\
\hline$C\left(7^{\prime}\right)-C(7)-C\left(11^{\prime}\right)$ & $165.7(10)$ \\
\hline$C(6)-C(7)-C\left(11^{\prime}\right)$ & $74.3(5)$ \\
\hline $\mathrm{C}(3)-\mathrm{C}(7)-\mathrm{C}\left(11^{\prime}\right)$ & $172.1(7)$ \\
\hline$C\left(6^{\prime}\right)-C(7)-C(12)$ & $132.3(7)$ \\
\hline$C\left(7^{\prime}\right)-C(7)-C(12)$ & $119.4(4)$ \\
\hline$C(6)-C(7)-C(12)$ & 126.0 \\
\hline$C(3)-C(7)-C(12)$ & 126.0 \\
\hline$C\left(11^{\prime}\right)-C(7)-C(12)$ & $52.3(5)$ \\
\hline$C\left(6^{\prime}\right)-C(7)-C\left(5^{\prime}\right)$ & $18.1(6)$ \\
\hline$C\left(7^{\prime}\right)-C(7)-C\left(5^{\prime}\right)$ & $91.1(4)$ \\
\hline $\mathrm{C}(6)-\mathrm{C}(7)-\mathrm{C}\left(5^{\prime}\right)$ & $23.5(2)$ \\
\hline $\mathrm{C}(3)-\mathrm{C}(7)-\mathrm{C}\left(5^{\prime}\right)$ & $84.8(2)$ \\
\hline $\mathrm{C}\left(11^{\prime}\right)-\mathrm{C}(7)-\mathrm{C}\left(5^{\prime}\right)$ & $96.8(5)$ \\
\hline$C(12)-C(7)-C\left(5^{\prime}\right)$ & $149.0(2)$ \\
\hline $\mathrm{C}\left(6^{\prime}\right)-\mathrm{C}(7)-\mathrm{Th}(1)$ & $84.3(10)$ \\
\hline$C\left(7^{\prime}\right)-C(7)-T h(1)$ & $80.7(5)$ \\
\hline$C(6)-C(7)-T h(1)$ & $74.86(19)$ \\
\hline$C(3)-C(7)-T h(1)$ & $74.67(19)$ \\
\hline$C\left(11^{\prime}\right)-C(7)-T h(1)$ & $113.2(7)$ \\
\hline$C(12)-C(7)-T h(1)$ & $116.55(19)$ \\
\hline $\mathrm{C}\left(5^{\prime}\right)-\mathrm{C}(7)-\mathrm{Th}(1)$ & $70.9(3)$ \\
\hline $\mathrm{C}\left(12^{\prime}\right)-\mathrm{C}(8)-\mathrm{C}(3)$ & $76.1(4)$ \\
\hline $\mathrm{C}\left(12^{\prime}\right)-\mathrm{C}(8)-\mathrm{C}\left(7^{\prime}\right)$ & $64.8(5)$ \\
\hline $\mathrm{C}(3)-\mathrm{C}(8)-\mathrm{C}\left(7^{\prime}\right)$ & $11.5(3)$ \\
\hline
\end{tabular}




\begin{tabular}{|c|c|}
\hline $\mathrm{C}\left(8^{\prime}\right)-\mathrm{C}(9)-\mathrm{C}\left(3^{\prime}\right)$ & $64.2(5)$ \\
\hline $\mathrm{C}\left(8^{\prime}\right)-\mathrm{C}(9)-\mathrm{C}(4)$ & $87.6(4)$ \\
\hline $\mathrm{C}\left(3^{\prime}\right)-\mathrm{C}(9)-\mathrm{C}(4)$ & $23.5(3)$ \\
\hline C(8')-C(9)-C(4') & $115.7(6)$ \\
\hline $\mathrm{C}\left(3^{\prime}\right)-\mathrm{C}(9)-\mathrm{C}\left(4^{\prime}\right)$ & $51.6(2)$ \\
\hline $\mathrm{C}(4)-\mathrm{C}(9)-\mathrm{C}\left(4^{\prime}\right)$ & $28.2(2)$ \\
\hline $\mathrm{C}\left(8^{\prime}\right)-\mathrm{C}(9)-\mathrm{C}\left(9^{\prime}\right)$ & $164.8(6)$ \\
\hline $\mathrm{C}\left(3^{\prime}\right)-\mathrm{C}(9)-\mathrm{C}\left(9^{\prime}\right)$ & $100.7(5)$ \\
\hline $\mathrm{C}(4)-\mathrm{C}(9)-\mathrm{C}\left(9^{\prime}\right)$ & $77.3(3)$ \\
\hline $\mathrm{C}\left(4^{\prime}\right)-\mathrm{C}(9)-\mathrm{C}\left(9^{\prime}\right)$ & 49.1(3) \\
\hline $\mathrm{C}(5)-\mathrm{C}(10)-\mathrm{C}\left(9^{\prime}\right)$ & $80.9(3)$ \\
\hline $\mathrm{C}(5)-\mathrm{C}(10)-\mathrm{C}\left(10^{\prime}\right)$ & $68.3(3)$ \\
\hline $\mathrm{C}\left(9^{\prime}\right)-\mathrm{C}(10)-\mathrm{C}\left(10^{\prime}\right)$ & $148.5(5)$ \\
\hline $\mathrm{C}(5)-\mathrm{C}(10)-\mathrm{C}\left(4^{\prime}\right)$ & $29.2(2)$ \\
\hline $\mathrm{C}\left(9^{\prime}\right)-\mathrm{C}(10)-\mathrm{C}\left(4^{\prime}\right)$ & $51.7(3)$ \\
\hline $\mathrm{C}\left(10^{\prime}\right)-\mathrm{C}(10)-\mathrm{C}\left(4^{\prime}\right)$ & $97.2(4)$ \\
\hline $\mathrm{C}(5)-\mathrm{C}(10)-\mathrm{C}\left(5^{\prime}\right)$ & $17.4(2)$ \\
\hline $\mathrm{C}\left(9^{\prime}\right)-\mathrm{C}(10)-\mathrm{C}\left(5^{\prime}\right)$ & $97.9(4)$ \\
\hline $\mathrm{C}\left(10^{\prime}\right)-\mathrm{C}(10)-\mathrm{C}\left(5^{\prime}\right)$ & $51.0(3)$ \\
\hline$C\left(4^{\prime}\right)-C(10)-C\left(5^{\prime}\right)$ & $46.21(18)$ \\
\hline $\mathrm{C}(6)-\mathrm{C}(11)-\mathrm{C}\left(10^{\prime}\right)$ & $68.7(3)$ \\
\hline $\mathrm{C}(6)-\mathrm{C}(11)-\mathrm{C}\left(11^{\prime}\right)$ & $60.2(2)$ \\
\hline $\mathrm{C}\left(10^{\prime}\right)-\mathrm{C}(11)-\mathrm{C}\left(11^{\prime}\right)$ & $127.3(5)$ \\
\hline$C\left(11^{\prime}\right)-C(12)-C(7)$ & $63.1(3)$ \\
\hline$C\left(11^{\prime}\right)-C(12)-C\left(6^{\prime}\right)$ & $50.3(3)$ \\
\hline $\mathrm{C}(7)-\mathrm{C}(12)-\mathrm{C}\left(6^{\prime}\right)$ & $13.0(2)$ \\
\hline $\mathrm{C}(4)-\mathrm{C}\left(3^{\prime}\right)-\mathrm{C}(3)$ & $106.2(10)$ \\
\hline $\mathrm{C}(4)-\mathrm{C}\left(3^{\prime}\right)-\mathrm{C}\left(4^{\prime}\right)$ & $7.8(8)$ \\
\hline $\mathrm{C}(3)-\mathrm{C}\left(3^{\prime}\right)-\mathrm{C}\left(4^{\prime}\right)$ & $113.2(4)$ \\
\hline $\mathrm{C}(4)-\mathrm{C}\left(3^{\prime}\right)-\mathrm{C}\left(7^{\prime}\right)$ & $101.2(7)$ \\
\hline $\mathrm{C}(3)-\mathrm{C}\left(3^{\prime}\right)-\mathrm{C}\left(7^{\prime}\right)$ & $6.1(5)$ \\
\hline $\mathrm{C}\left(4^{\prime}\right)-\mathrm{C}\left(3^{\prime}\right)-\mathrm{C}\left(7^{\prime}\right)$ & 108.0 \\
\hline C(4)-C(3')-C(9) & $79.8(8)$ \\
\hline $\mathrm{C}(3)-\mathrm{C}\left(3^{\prime}\right)-\mathrm{C}(9)$ & $173.4(8)$ \\
\hline $\mathrm{C}\left(4^{\prime}\right)-\mathrm{C}\left(3^{\prime}\right)-\mathrm{C}(9)$ & $73.0(4)$ \\
\hline $\mathrm{C}\left(7^{\prime}\right)-\mathrm{C}\left(3^{\prime}\right)-\mathrm{C}(9)$ & $178.6(5)$ \\
\hline
\end{tabular}




\begin{tabular}{|c|c|}
\hline $\mathrm{C}(4)-\mathrm{C}\left(3^{\prime}\right)-\mathrm{C}\left(8^{\prime}\right)$ & $132.6(7)$ \\
\hline $\mathrm{C}(3)-\mathrm{C}\left(3^{\prime}\right)-\mathrm{C}\left(8^{\prime}\right)$ & $120.7(4)$ \\
\hline $\mathrm{C}\left(4^{\prime}\right)-\mathrm{C}\left(3^{\prime}\right)-\mathrm{C}\left(8^{\prime}\right)$ & 126.0 \\
\hline $\mathrm{C}\left(7^{\prime}\right)-\mathrm{C}\left(3^{\prime}\right)-\mathrm{C}\left(8^{\prime}\right)$ & 126.0 \\
\hline $\mathrm{C}(9)-\mathrm{C}\left(3^{\prime}\right)-\mathrm{C}\left(8^{\prime}\right)$ & $53.0(4)$ \\
\hline $\mathrm{C}(4)-\mathrm{C}\left(3^{\prime}\right)-\mathrm{C}(5)$ & $16.0(6)$ \\
\hline $\mathrm{C}(3)-\mathrm{C}\left(3^{\prime}\right)-\mathrm{C}(5)$ & $90.4(4)$ \\
\hline$C\left(4^{\prime}\right)-C\left(3^{\prime}\right)-C(5)$ & $22.9(2)$ \\
\hline$C\left(7^{\prime}\right)-C\left(3^{\prime}\right)-C(5)$ & $85.3(2)$ \\
\hline$C(9)-C\left(3^{\prime}\right)-C(5)$ & $95.8(4)$ \\
\hline $\mathrm{C}\left(8^{\prime}\right)-\mathrm{C}\left(3^{\prime}\right)-\mathrm{C}(5)$ & $148.6(2)$ \\
\hline $\mathrm{C}(4)-\mathrm{C}\left(3^{\prime}\right)-\mathrm{Th}(1)$ & $69.8(10)$ \\
\hline $\mathrm{C}(3)-\mathrm{C}\left(3^{\prime}\right)-\mathrm{Th}(1)$ & $72.3(5)$ \\
\hline $\mathrm{C}\left(4^{\prime}\right)-\mathrm{C}\left(3^{\prime}\right)-\mathrm{Th}(1)$ & $75.9(2)$ \\
\hline $\mathrm{C}\left(7^{\prime}\right)-\mathrm{C}\left(3^{\prime}\right)-\mathrm{Th}(1)$ & $73.3(2)$ \\
\hline $\mathrm{C}(9)-\mathrm{C}\left(3^{\prime}\right)-\mathrm{Th}(1)$ & $108.0(5)$ \\
\hline $\mathrm{C}\left(8^{\prime}\right)-\mathrm{C}\left(3^{\prime}\right)-\mathrm{Th}(1)$ & $116.9(2)$ \\
\hline $\mathrm{C}(5)-\mathrm{C}\left(3^{\prime}\right)-\mathrm{Th}(1)$ & $65.6(3)$ \\
\hline $\mathrm{C}(4)-\mathrm{C}\left(4^{\prime}\right)-\mathrm{C}(5)$ & $113.1(8)$ \\
\hline $\mathrm{C}(4)-\mathrm{C}\left(4^{\prime}\right)-\mathrm{C}\left(3^{\prime}\right)$ & $5.8(6)$ \\
\hline $\mathrm{C}(5)-\mathrm{C}\left(4^{\prime}\right)-\mathrm{C}\left(3^{\prime}\right)$ & $118.4(5)$ \\
\hline $\mathrm{C}(4)-\mathrm{C}\left(4^{\prime}\right)-\mathrm{C}\left(5^{\prime}\right)$ & $103.0(5)$ \\
\hline $\mathrm{C}(5)-\mathrm{C}\left(4^{\prime}\right)-\mathrm{C}\left(5^{\prime}\right)$ & $12.3(5)$ \\
\hline $\mathrm{C}\left(3^{\prime}\right)-\mathrm{C}\left(4^{\prime}\right)-\mathrm{C}\left(5^{\prime}\right)$ & 108.0 \\
\hline $\mathrm{C}(4)-\mathrm{C}\left(4^{\prime}\right)-\mathrm{C}\left(9^{\prime}\right)$ & $130.9(5)$ \\
\hline $\mathrm{C}(5)-\mathrm{C}\left(4^{\prime}\right)-\mathrm{C}\left(9^{\prime}\right)$ & $115.2(5)$ \\
\hline $\mathrm{C}\left(3^{\prime}\right)-\mathrm{C}\left(4^{\prime}\right)-\mathrm{C}\left(9^{\prime}\right)$ & 126.0 \\
\hline $\mathrm{C}\left(5^{\prime}\right)-\mathrm{C}\left(4^{\prime}\right)-\mathrm{C}\left(9^{\prime}\right)$ & 126.0 \\
\hline $\mathrm{C}(4)-\mathrm{C}\left(4^{\prime}\right)-\mathrm{C}(9)$ & $60.5(5)$ \\
\hline $\mathrm{C}(5)-\mathrm{C}\left(4^{\prime}\right)-\mathrm{C}(9)$ & $172.0(9)$ \\
\hline $\mathrm{C}\left(3^{\prime}\right)-\mathrm{C}\left(4^{\prime}\right)-\mathrm{C}(9)$ & $55.4(4)$ \\
\hline$C\left(5^{\prime}\right)-\mathrm{C}\left(4^{\prime}\right)-\mathrm{C}(9)$ & $163.4(4)$ \\
\hline $\mathrm{C}\left(9^{\prime}\right)-\mathrm{C}\left(4^{\prime}\right)-\mathrm{C}(9)$ & $70.6(4)$ \\
\hline$C(4)-C\left(4^{\prime}\right)-C(10)$ & $168.7(9)$ \\
\hline$C(5)-C\left(4^{\prime}\right)-C(10)$ & $56.6(4)$ \\
\hline$C\left(3^{\prime}\right)-C\left(4^{\prime}\right)-C(10)$ & $174.5(4)$ \\
\hline
\end{tabular}




\begin{tabular}{|c|c|}
\hline$C\left(5^{\prime}\right)-C\left(4^{\prime}\right)-C(10)$ & $67.4(4)$ \\
\hline $\mathrm{C}\left(9^{\prime}\right)-\mathrm{C}\left(4^{\prime}\right)-\mathrm{C}(10)$ & $58.7(4)$ \\
\hline$C(9)-C\left(4^{\prime}\right)-C(10)$ & $129.2(4)$ \\
\hline$C(4)-C\left(4^{\prime}\right)-\operatorname{Th}(1)$ & 71.4(7) \\
\hline$C(5)-C\left(4^{\prime}\right)-\operatorname{Th}(1)$ & $71.7(6)$ \\
\hline $\mathrm{C}\left(3^{\prime}\right)-\mathrm{C}\left(4^{\prime}\right)-\mathrm{Th}(1)$ & $76.0(2)$ \\
\hline $\mathrm{C}\left(5^{\prime}\right)-\mathrm{C}\left(4^{\prime}\right)-\mathrm{Th}(1)$ & $73.2(2)$ \\
\hline $\mathrm{C}\left(9^{\prime}\right)-\mathrm{C}\left(4^{\prime}\right)-\mathrm{Th}(1)$ & $116.8(2)$ \\
\hline$C(9)-C\left(4^{\prime}\right)-\operatorname{Th}(1)$ & $101.0(5)$ \\
\hline $\mathrm{C}(10)-\mathrm{C}\left(4^{\prime}\right)-\mathrm{Th}(1)$ & $99.4(4)$ \\
\hline$C(5)-C\left(5^{\prime}\right)-C(6)$ & $150.7(13)$ \\
\hline $\mathrm{C}(5)-\mathrm{C}\left(5^{\prime}\right)-\mathrm{C}\left(6^{\prime}\right)$ & $123.7(7)$ \\
\hline $\mathrm{C}(6)-\mathrm{C}\left(5^{\prime}\right)-\mathrm{C}\left(6^{\prime}\right)$ & $34.7(6)$ \\
\hline $\mathrm{C}(5)-\mathrm{C}\left(5^{\prime}\right)-\mathrm{C}\left(4^{\prime}\right)$ & $18.6(8)$ \\
\hline $\mathrm{C}(6)-\mathrm{C}\left(5^{\prime}\right)-\mathrm{C}\left(4^{\prime}\right)$ & $140.5(5)$ \\
\hline $\mathrm{C}\left(6^{\prime}\right)-\mathrm{C}\left(5^{\prime}\right)-\mathrm{C}\left(4^{\prime}\right)$ & 108.0 \\
\hline $\mathrm{C}(5)-\mathrm{C}\left(5^{\prime}\right)-\mathrm{C}\left(10^{\prime}\right)$ & $109.6(7)$ \\
\hline $\mathrm{C}(6)-\mathrm{C}\left(5^{\prime}\right)-\mathrm{C}\left(10^{\prime}\right)$ & $92.5(5)$ \\
\hline $\mathrm{C}\left(6^{\prime}\right)-\mathrm{C}\left(5^{\prime}\right)-\mathrm{C}\left(10^{\prime}\right)$ & 126.0 \\
\hline$C\left(4^{\prime}\right)-C\left(5^{\prime}\right)-C\left(10^{\prime}\right)$ & 126.0 \\
\hline $\mathrm{C}(5)-\mathrm{C}\left(5^{\prime}\right)-\mathrm{C}(4)$ & $43.1(6)$ \\
\hline$C(6)-C\left(5^{\prime}\right)-C(4)$ & $114.4(6)$ \\
\hline$C\left(6^{\prime}\right)-C\left(5^{\prime}\right)-C(4)$ & $81.6(2)$ \\
\hline$C\left(4^{\prime}\right)-C\left(5^{\prime}\right)-C(4)$ & $26.5(3)$ \\
\hline $\mathrm{C}\left(10^{\prime}\right)-\mathrm{C}\left(5^{\prime}\right)-\mathrm{C}(4)$ & $152.4(2)$ \\
\hline$C(5)-C\left(5^{\prime}\right)-C(10)$ & $50.2(6)$ \\
\hline $\mathrm{C}(6)-\mathrm{C}\left(5^{\prime}\right)-\mathrm{C}(10)$ & $149.5(8)$ \\
\hline $\mathrm{C}\left(6^{\prime}\right)-\mathrm{C}\left(5^{\prime}\right)-\mathrm{C}(10)$ & $173.6(4)$ \\
\hline$C\left(4^{\prime}\right)-C\left(5^{\prime}\right)-C(10)$ & $66.4(4)$ \\
\hline $\mathrm{C}\left(10^{\prime}\right)-\mathrm{C}\left(5^{\prime}\right)-\mathrm{C}(10)$ & $59.7(4)$ \\
\hline$C(4)-C\left(5^{\prime}\right)-C(10)$ & $92.7(4)$ \\
\hline$C(5)-C\left(5^{\prime}\right)-C(7)$ & $116.6(8)$ \\
\hline $\mathrm{C}(6)-\mathrm{C}\left(5^{\prime}\right)-\mathrm{C}(7)$ & $40.1(4)$ \\
\hline$C\left(6^{\prime}\right)-C\left(5^{\prime}\right)-C(7)$ & $7.5(3)$ \\
\hline$C\left(4^{\prime}\right)-C\left(5^{\prime}\right)-C(7)$ & $101.5(3)$ \\
\hline$C\left(10^{\prime}\right)-C\left(5^{\prime}\right)-C(7)$ & $132.4(3)$ \\
\hline
\end{tabular}




\begin{tabular}{|c|c|}
\hline $\mathrm{C}(4)-\mathrm{C}\left(5^{\prime}\right)-\mathrm{C}(7)$ & $75.0(3)$ \\
\hline$C(10)-C\left(5^{\prime}\right)-C(7)$ & $166.1(5)$ \\
\hline$C(5)-C\left(5^{\prime}\right)-\operatorname{Th}(1)$ & 76.1(9) \\
\hline $\mathrm{C}(6)-\mathrm{C}\left(5^{\prime}\right)-\mathrm{Th}(1)$ & $77.4(6)$ \\
\hline $\mathrm{C}\left(6^{\prime}\right)-\mathrm{C}\left(5^{\prime}\right)-\mathrm{Th}(1)$ & $73.9(2)$ \\
\hline $\mathrm{C}\left(4^{\prime}\right)-\mathrm{C}\left(5^{\prime}\right)-\mathrm{Th}(1)$ & $78.4(2)$ \\
\hline $\mathrm{C}\left(10^{\prime}\right)-\mathrm{C}\left(5^{\prime}\right)-\mathrm{Th}(1)$ & $114.0(2)$ \\
\hline $\mathrm{C}(4)-\mathrm{C}\left(5^{\prime}\right)-\mathrm{Th}(1)$ & $68.9(3)$ \\
\hline$C(10)-C\left(5^{\prime}\right)-\operatorname{Th}(1)$ & $101.4(4)$ \\
\hline$C(7)-C\left(5^{\prime}\right)-\operatorname{Th}(1)$ & $68.4(3)$ \\
\hline $\mathrm{C}(7)-\mathrm{C}\left(6^{\prime}\right)-\mathrm{C}(6)$ & $154.8(17)$ \\
\hline $\mathrm{C}(7)-\mathrm{C}\left(6^{\prime}\right)-\mathrm{C}\left(5^{\prime}\right)$ & $154.5(9)$ \\
\hline $\mathrm{C}(6)-\mathrm{C}\left(6^{\prime}\right)-\mathrm{C}\left(5^{\prime}\right)$ & $35.6(6)$ \\
\hline $\mathrm{C}(7)-\mathrm{C}\left(6^{\prime}\right)-\mathrm{C}\left(7^{\prime}\right)$ & $50.6(9)$ \\
\hline $\mathrm{C}(6)-\mathrm{C}\left(6^{\prime}\right)-\mathrm{C}\left(7^{\prime}\right)$ & $141.2(5)$ \\
\hline $\mathrm{C}\left(5^{\prime}\right)-\mathrm{C}\left(6^{\prime}\right)-\mathrm{C}\left(7^{\prime}\right)$ & 108.0 \\
\hline$C(7)-C\left(6^{\prime}\right)-C\left(11^{\prime}\right)$ & $76.8(8)$ \\
\hline $\mathrm{C}(6)-\mathrm{C}\left(6^{\prime}\right)-\mathrm{C}\left(11^{\prime}\right)$ & $91.6(5)$ \\
\hline $\mathrm{C}\left(5^{\prime}\right)-\mathrm{C}\left(6^{\prime}\right)-\mathrm{C}\left(11^{\prime}\right)$ & 126.0 \\
\hline $\mathrm{C}\left(7^{\prime}\right)-\mathrm{C}\left(6^{\prime}\right)-\mathrm{C}\left(11^{\prime}\right)$ & 126.0 \\
\hline $\mathrm{C}(7)-\mathrm{C}\left(6^{\prime}\right)-\mathrm{C}(3)$ & $58.9(7)$ \\
\hline $\mathrm{C}(6)-\mathrm{C}\left(6^{\prime}\right)-\mathrm{C}(3)$ & $131.9(7)$ \\
\hline$C\left(5^{\prime}\right)-C\left(6^{\prime}\right)-C(3)$ & $98.9(3)$ \\
\hline$C\left(7^{\prime}\right)-C\left(6^{\prime}\right)-C(3)$ & $9.3(3)$ \\
\hline $\mathrm{C}\left(11^{\prime}\right)-\mathrm{C}\left(6^{\prime}\right)-\mathrm{C}(3)$ & $135.0(3)$ \\
\hline $\mathrm{C}(7)-\mathrm{C}\left(6^{\prime}\right)-\mathrm{C}(5)$ & $139.3(10)$ \\
\hline $\mathrm{C}(6)-\mathrm{C}\left(6^{\prime}\right)-\mathrm{C}(5)$ & $49.8(4)$ \\
\hline$C\left(5^{\prime}\right)-C\left(6^{\prime}\right)-C(5)$ & $15.7(2)$ \\
\hline$C\left(7^{\prime}\right)-C\left(6^{\prime}\right)-C(5)$ & $92.6(2)$ \\
\hline $\mathrm{C}\left(11^{\prime}\right)-\mathrm{C}\left(6^{\prime}\right)-\mathrm{C}(5)$ & $141.3(2)$ \\
\hline $\mathrm{C}(3)-\mathrm{C}\left(6^{\prime}\right)-\mathrm{C}(5)$ & $83.4(3)$ \\
\hline$C(7)-C\left(6^{\prime}\right)-C(12)$ & $34.8(6)$ \\
\hline$C(6)-C\left(6^{\prime}\right)-C(12)$ & $131.6(8)$ \\
\hline$C\left(5^{\prime}\right)-C\left(6^{\prime}\right)-C(12)$ & $167.1(5)$ \\
\hline$C\left(7^{\prime}\right)-C\left(6^{\prime}\right)-C(12)$ & $83.9(4)$ \\
\hline$C\left(11^{\prime}\right)-C\left(6^{\prime}\right)-C(12)$ & $42.4(4)$ \\
\hline
\end{tabular}




\begin{tabular}{|c|c|}
\hline$C(3)-C\left(6^{\prime}\right)-C(12)$ & $92.7(4)$ \\
\hline$C(5)-C\left(6^{\prime}\right)-C(12)$ & $171.3(6)$ \\
\hline$C(7)-C\left(6^{\prime}\right)-\operatorname{Th}(1)$ & $83.6(10)$ \\
\hline$C(6)-C\left(6^{\prime}\right)-\operatorname{Th}(1)$ & $80.1(7)$ \\
\hline $\mathrm{C}\left(5^{\prime}\right)-\mathrm{C}\left(6^{\prime}\right)-\mathrm{Th}(1)$ & 77.1(2) \\
\hline$C\left(7^{\prime}\right)-C\left(6^{\prime}\right)-T h(1)$ & $77.2(2)$ \\
\hline$C\left(11^{\prime}\right)-C\left(6^{\prime}\right)-\operatorname{Th}(1)$ & $112.3(2)$ \\
\hline$C(3)-C\left(6^{\prime}\right)-\operatorname{Th}(1)$ & $72.4(4)$ \\
\hline $\mathrm{C}(5)-\mathrm{C}\left(6^{\prime}\right)-\mathrm{Th}(1)$ & $69.9(3)$ \\
\hline$C(12)-C\left(6^{\prime}\right)-T h(1)$ & $101.6(5)$ \\
\hline $\mathrm{C}(7)-\mathrm{C}\left(7^{\prime}\right)-\mathrm{C}\left(6^{\prime}\right)$ & $23.8(4)$ \\
\hline $\mathrm{C}(7)-\mathrm{C}\left(7^{\prime}\right)-\mathrm{C}\left(3^{\prime}\right)$ & $130.7(4)$ \\
\hline $\mathrm{C}\left(6^{\prime}\right)-\mathrm{C}\left(7^{\prime}\right)-\mathrm{C}\left(3^{\prime}\right)$ & 108.0 \\
\hline $\mathrm{C}(7)-\mathrm{C}\left(7^{\prime}\right)-\mathrm{C}\left(12^{\prime}\right)$ & $102.9(4)$ \\
\hline $\mathrm{C}\left(6^{\prime}\right)-\mathrm{C}\left(7^{\prime}\right)-\mathrm{C}\left(12^{\prime}\right)$ & 126.0 \\
\hline $\mathrm{C}\left(3^{\prime}\right)-\mathrm{C}\left(7^{\prime}\right)-\mathrm{C}\left(12^{\prime}\right)$ & 126.0 \\
\hline $\mathrm{C}(7)-\mathrm{C}\left(7^{\prime}\right)-\mathrm{C}(8)$ & $143.2(7)$ \\
\hline $\mathrm{C}\left(6^{\prime}\right)-\mathrm{C}\left(7^{\prime}\right)-\mathrm{C}(8)$ & $166.7(5)$ \\
\hline $\mathrm{C}\left(3^{\prime}\right)-\mathrm{C}\left(7^{\prime}\right)-\mathrm{C}(8)$ & $85.2(5)$ \\
\hline $\mathrm{C}\left(12^{\prime}\right)-\mathrm{C}\left(7^{\prime}\right)-\mathrm{C}(8)$ & $40.8(5)$ \\
\hline $\mathrm{C}(7)-\mathrm{C}\left(7^{\prime}\right)-\mathrm{C}(4)$ & $109.4(5)$ \\
\hline $\mathrm{C}\left(6^{\prime}\right)-\mathrm{C}\left(7^{\prime}\right)-\mathrm{C}(4)$ & $86.8(3)$ \\
\hline $\mathrm{C}\left(3^{\prime}\right)-\mathrm{C}\left(7^{\prime}\right)-\mathrm{C}(4)$ & 21.3(3) \\
\hline $\mathrm{C}\left(12^{\prime}\right)-\mathrm{C}\left(7^{\prime}\right)-\mathrm{C}(4)$ & $147.2(3)$ \\
\hline $\mathrm{C}(8)-\mathrm{C}\left(7^{\prime}\right)-\mathrm{C}(4)$ & $106.4(5)$ \\
\hline$C(7)-C\left(7^{\prime}\right)-T h(1)$ & $76.2(5)$ \\
\hline $\mathrm{C}\left(6^{\prime}\right)-\mathrm{C}\left(7^{\prime}\right)-\mathrm{Th}(1)$ & $73.9(2)$ \\
\hline $\mathrm{C}\left(3^{\prime}\right)-\mathrm{C}\left(7^{\prime}\right)-\mathrm{Th}(1)$ & $78.4(2)$ \\
\hline$C\left(12^{\prime}\right)-C\left(7^{\prime}\right)-\operatorname{Th}(1)$ & $114.1(2)$ \\
\hline $\mathrm{C}(8)-\mathrm{C}\left(7^{\prime}\right)-\mathrm{Th}(1)$ & 109.2(6) \\
\hline$C(4)-C\left(7^{\prime}\right)-\operatorname{Th}(1)$ & 70.1(4) \\
\hline $\mathrm{C}(9)-\mathrm{C}\left(8^{\prime}\right)-\mathrm{C}\left(3^{\prime}\right)$ & $62.8(3)$ \\
\hline C(9)-C(8')-C(4) & $49.8(2)$ \\
\hline $\mathrm{C}\left(3^{\prime}\right)-\mathrm{C}\left(8^{\prime}\right)-\mathrm{C}(4)$ & $13.2(2)$ \\
\hline$C\left(4^{\prime}\right)-C\left(9^{\prime}\right)-C(10)$ & $69.6(3)$ \\
\hline $\mathrm{C}\left(4^{\prime}\right)-\mathrm{C}\left(9^{\prime}\right)-\mathrm{C}(9)$ & $60.3(2)$ \\
\hline
\end{tabular}




$\begin{array}{lr}\mathrm{C}(10)-\mathrm{C}\left(9^{\prime}\right)-\mathrm{C}(9) & 129.8(4) \\ \mathrm{C}\left(5^{\prime}\right)-\mathrm{C}\left(10^{\prime}\right)-\mathrm{C}(11) & 81.7(3) \\ \mathrm{C}\left(5^{\prime}\right)-\mathrm{C}\left(10^{\prime}\right)-\mathrm{C}(10) & 69.3(3) \\ \mathrm{C}(11)-\mathrm{C}\left(10^{\prime}\right)-\mathrm{C}(10) & 149.0(5) \\ \mathrm{C}\left(5^{\prime}\right)-\mathrm{C}\left(10^{\prime}\right)-\mathrm{C}(6) & 29.5(3) \\ \mathrm{C}(11)-\mathrm{C}\left(10^{\prime}\right)-\mathrm{C}(6) & 52.2(3) \\ \mathrm{C}(10)-\mathrm{C}\left(10^{\prime}\right)-\mathrm{C}(6) & 98.1(4) \\ \mathrm{C}\left(5^{\prime}\right)-\mathrm{C}\left(10^{\prime}\right)-\mathrm{C}(5) & 18.0(3) \\ \mathrm{C}(11)-\mathrm{C}\left(10^{\prime}\right)-\mathrm{C}(5) & 98.9(5) \\ \mathrm{C}(10)-\mathrm{C}\left(10^{\prime}\right)-\mathrm{C}(5) & 51.4(3) \\ \mathrm{C}(6)-\mathrm{C}\left(10^{\prime}\right)-\mathrm{C}(5) & 46.84(18) \\ \mathrm{C}(12)-\mathrm{C}\left(11^{\prime}\right)-\mathrm{C}(7) & 64.6(5) \\ \mathrm{C}(12)-\mathrm{C}\left(11^{\prime}\right)-\mathrm{C}\left(6^{\prime}\right) & 87.3(4) \\ \mathrm{C}(7)-\mathrm{C}\left(11^{\prime}\right)-\mathrm{C}\left(6^{\prime}\right) & 22.9(3) \\ \mathrm{C}(12)-\mathrm{C}\left(11^{\prime}\right)-\mathrm{C}(6) & 115.2(6) \\ \mathrm{C}(7)-\mathrm{C}\left(11^{\prime}\right)-\mathrm{C}(6) & 51.0(2) \\ \mathrm{C}\left(6^{\prime}\right)-\mathrm{C}\left(11^{\prime}\right)-\mathrm{C}(6) & 29.2(3) \\ \mathrm{C}(12)-\mathrm{C}\left(11^{\prime}\right)-\mathrm{C}(11) & 159.3(9) \\ \mathrm{C}(7)-\mathrm{C}\left(11^{\prime}\right)-\mathrm{C}(11) & 98.9(5) \\ \mathrm{C}\left(6^{\prime}\right)-\mathrm{C}\left(11^{\prime}\right)-\mathrm{C}(11) & 77.3(3) \\ \mathrm{C}(6)-\mathrm{C}\left(11^{\prime}\right)-\mathrm{C}(11) & 48.2(3) \\ \mathrm{C}(8)-\mathrm{C}\left(12^{\prime}\right)-\mathrm{C}\left(7^{\prime}\right) & 74.5(4) \\ \mathrm{C}(8)-\mathrm{C}\left(12^{\prime}\right)-\mathrm{C}(3) & 63.6(4) \\ \mathrm{C}\left(7^{\prime}\right)-\mathrm{C}\left(12^{\prime}\right)-\mathrm{C}(3) & 11.0(3) \\ & \end{array}$

Symmetry transformations used to generate equivalent atoms:

\#1 x,-y+3/2,z 
Table S9. Anisotropic displacement parameters $\left(\AA^{2} \times 10^{3}\right)$ for $\left(\mathrm{C}_{5} \mathrm{Me}_{5}\right)_{2} \mathrm{Th}\left(\mathrm{CH}_{3}\right)_{2}(\mathbf{9 c})$. The anisotropic displacement factor exponent takes the form: $-2 \pi^{2}\left[h^{2} a^{* 2} U^{11}+\ldots+2 h k a^{*} b^{*} U^{12}\right]$

\begin{tabular}{|c|c|c|c|c|c|c|}
\hline & $\mathrm{U}^{11}$ & $\mathrm{U}^{22}$ & $\mathrm{U}^{33}$ & $\mathrm{U}^{23}$ & $\mathrm{U}^{13}$ & $\mathrm{U}^{12}$ \\
\hline $\operatorname{Th}(1)$ & $32(1)$ & $32(1)$ & $32(1)$ & 0 & $14(1)$ & 0 \\
\hline$C(1)$ & $63(5)$ & $53(6)$ & $60(5)$ & 0 & $40(5)$ & 0 \\
\hline$C(2)$ & $37(4)$ & $56(6)$ & $71(6)$ & 0 & $4(4)$ & 0 \\
\hline$C(3)$ & $47(7)$ & $38(8)$ & $36(7)$ & $-15(6)$ & $20(6)$ & $0(6)$ \\
\hline$C(4)$ & $45(8)$ & $24(5)$ & $58(11)$ & $-13(6)$ & $29(9)$ & $-15(5)$ \\
\hline$C(5)$ & $62(10)$ & $36(7)$ & $28(6)$ & $10(5)$ & $26(6)$ & $23(7)$ \\
\hline$C(6)$ & $35(6)$ & $42(6)$ & $39(6)$ & $-2(5)$ & $20(5)$ & $9(5)$ \\
\hline$C(7)$ & $49(7)$ & $40(6)$ & $28(6)$ & $-3(5)$ & $20(5)$ & $3(5)$ \\
\hline$C(8)$ & $77(12)$ & $66(11)$ & $65(10)$ & $-22(9)$ & $14(8)$ & $-14(9)$ \\
\hline $\mathrm{C}(9)$ & $82(10)$ & $43(8)$ & $140(16)$ & $-1(8)$ & $80(11)$ & $-15(7)$ \\
\hline$C(10)$ & $116(14)$ & $86(12)$ & $39(7)$ & $23(7)$ & $43(8)$ & $26(11)$ \\
\hline $\mathrm{C}(11)$ & $48(8)$ & $79(11)$ & $57(8)$ & $-4(7)$ & $4(6)$ & $13(7)$ \\
\hline$C(12)$ & $85(11)$ & $67(9)$ & $65(8)$ & $1(7)$ & $54(8)$ & $5(8)$ \\
\hline$C\left(3^{\prime}\right)$ & $46(7)$ & $37(6)$ & $37(8)$ & $-12(5)$ & $15(7)$ & $-8(5)$ \\
\hline$C\left(4^{\prime}\right)$ & $64(10)$ & $36(6)$ & $37(6)$ & $-10(6)$ & $20(7)$ & $-4(6)$ \\
\hline$C\left(5^{\prime}\right)$ & $53(10)$ & $46(8)$ & $47(9)$ & $-10(6)$ & $10(7)$ & $11(7)$ \\
\hline$C\left(6^{\prime}\right)$ & $50(8)$ & $44(7)$ & $60(11)$ & $-15(7)$ & $38(8)$ & $-6(6)$ \\
\hline$C\left(7^{\prime}\right)$ & $55(8)$ & $36(8)$ & $42(8)$ & $10(6)$ & $27(7)$ & $8(6)$ \\
\hline$C\left(8^{\prime}\right)$ & $52(8)$ & $56(9)$ & $126(15)$ & $-25(10)$ & $39(9)$ & $-17(7)$ \\
\hline$C\left(9^{\prime}\right)$ & 131(16) & $62(10)$ & $83(11)$ & $12(8)$ & $79(11)$ & $14(10)$ \\
\hline$C\left(10^{\prime}\right)$ & $132(19)$ & $61(11)$ & $83(13)$ & $-20(10)$ & $-71(13)$ & $36(12)$ \\
\hline$C\left(11^{\prime}\right)$ & $150(20)$ & $92(17)$ & $460(60)$ & $80(30)$ & $240(40)$ & $46(17)$ \\
\hline$C\left(12^{\prime}\right)$ & $137(18)$ & $85(13)$ & $41(7)$ & $-9(7)$ & $44(10)$ & $28(12)$ \\
\hline
\end{tabular}


Table S10. Hydrogen coordinates ( $\left.x 10^{4}\right)$ and isotropic displacement parameters $\left(\AA^{2} \times 10^{3}\right)$ for $\left(\mathrm{C}_{5} \mathrm{Me}_{5}\right)_{2} \mathrm{Th}\left(\mathrm{CH}_{3}\right)_{2}(\mathbf{9 c})$.

\begin{tabular}{lrrrr}
\hline & $x$ & $y$ & $z$ & U(eq) \\
\hline $\mathrm{H}(1 \mathrm{~A})$ & 3392 & 7500 & 7402 & 81 \\
$\mathrm{H}(1 \mathrm{~B})$ & 1614 & 7047 & 6216 & 81 \\
$\mathrm{H}(1 \mathrm{C})$ & 1614 & 7953 & 6216 & 81 \\
$\mathrm{H}(2 \mathrm{~A})$ & 113 & 7500 & 234 & 94 \\
$\mathrm{H}(2 \mathrm{~B})$ & -595 & 7953 & 1391 & 94 \\
$\mathrm{H}(2 \mathrm{C})$ & -595 & 7047 & 1391 & 94 \\
& & & & \\
\hline
\end{tabular}


Table S11. Crystal data and structure refinement for $\left(\mathrm{C}_{5} \mathrm{Me}_{5}\right)_{2} \mathrm{Th}\left(-\mathrm{N}=\mathrm{CPh}_{2}\right)_{2}$ (10a).

Identification code

Empirical formula

Formula weight

Temperature

Wavelength

Crystal system

Space group

Unit cell dimensions

Volume

Z

Density (calculated)

Absorption coefficient

$\mathrm{F}(000)$

Crystal size

Theta range for data collection

Index ranges

Reflections collected

Independent reflections

Max. and min. transmission

Refinement method

Data / restraints / parameters

Goodness-of-fit on $\mathrm{F}^{2}$

Final $\mathrm{R}$ indices [I $>2 \operatorname{sigma}(\mathrm{I})]$

$\mathrm{R}$ indices (all data)

Largest diff. peak and hole $\operatorname{ccd} 776$

$\mathrm{C}_{46} \mathrm{H}_{50} \mathrm{~N}_{2} \mathrm{Th}$

862.92

203(2) K

$0.71073 \AA$

triclinic

P -1

$\mathrm{a}=10.640(4) \AA$

$\alpha=64.241(5)^{\circ}$.

$\mathrm{b}=20.378(7) \AA$

$\beta=82.931(6)^{\circ}$.

$\mathrm{c}=20.555(7) \AA$ $\gamma=82.307(7)^{\circ}$.

3967(2) $\AA^{3}$

4

$1.445 \mathrm{Mg} / \mathrm{m}^{3}$

$3.791 \mathrm{~mm}^{-1}$

1720

$0.40 \times 0.28 \times 0.26 \mathrm{~mm}^{3}$

1.10 to $23.26^{\circ}$.

$-11<=\mathrm{h}<=11,-22<=\mathrm{k}<=22,-22<=1<=22$

20868

$10444[\mathrm{R}(\mathrm{int})=0.0184]$

0.4390 and 0.3124

Full-matrix least-squares on $\mathrm{F}^{2}$

10444 / 0 / 883

2.092

$\mathrm{R} 1=0.0337, \mathrm{wR} 2=0.0601$

$\mathrm{R} 1=0.0437, \mathrm{wR} 2=0.0612$

0.678 and -0.770 e. $\AA^{-3}$ 
Table S12. Atomic coordinates ( x 104) and equivalent isotropic displacement parameters $\left(\AA^{2} \times 10^{3}\right)$ for $\left(\mathrm{C}_{5} \mathrm{Me}_{5}\right)_{2} \mathrm{Th}\left(-\mathrm{N}=\mathrm{CPh}_{2}\right)_{2}$ (10a). U(eq) is defined as one third of the trace of the orthogonalized $\mathrm{U}^{\mathrm{ij}}$ tensor.

\begin{tabular}{|c|c|c|c|c|}
\hline & $\mathrm{x}$ & $\mathrm{y}$ & $\mathrm{z}$ & $\mathrm{U}(\mathrm{eq})$ \\
\hline $\operatorname{Th}(1)$ & $1815(1)$ & $5250(1)$ & 2741(1) & $30(1)$ \\
\hline $\operatorname{Th}(2)$ & $15848(1)$ & $9707(1)$ & $2296(1)$ & $28(1)$ \\
\hline $\mathrm{N}(1)$ & $1635(5)$ & $4040(2)$ & $3157(3)$ & $40(1)$ \\
\hline $\mathrm{N}(2)$ & $482(5)$ & $5874(3)$ & $1847(3)$ & $43(1)$ \\
\hline $\mathrm{N}(3)$ & $15005(4)$ & $10877(2)$ & 1931(2) & $36(1)$ \\
\hline $\mathrm{N}(4)$ & $14538(5)$ & $9020(3)$ & $3224(2)$ & $42(1)$ \\
\hline $\mathrm{C}(1)$ & 1097(6) & $6007(3)$ & $3602(3)$ & $42(2)$ \\
\hline$C(2)$ & $1563(6)$ & $5285(4)$ & $4090(3)$ & $49(2)$ \\
\hline $\mathrm{C}(3)$ & $645(7)$ & $4807(3)$ & $4156(3)$ & $50(2)$ \\
\hline $\mathrm{C}(4)$ & $-335(6)$ & $5221(3)$ & $3708(3)$ & $44(2)$ \\
\hline$C(5)$ & $-73(6)$ & $5953(3)$ & $3376(3)$ & $41(2)$ \\
\hline$C(6)$ & $1676(7)$ & $6709(4)$ & $3427(4)$ & $71(2)$ \\
\hline$C(7)$ & $2710(7)$ & $5080(5)$ & $4506(4)$ & $81(2)$ \\
\hline $\mathrm{C}(8)$ & $665(8)$ & 4012(4) & $4696(4)$ & $81(3)$ \\
\hline$C(9)$ & $-1519(7)$ & 4938(4) & $3612(4)$ & $76(2)$ \\
\hline $\mathrm{C}(10)$ & $-988(6)$ & $6582(4)$ & 2955(4) & $62(2)$ \\
\hline $\mathrm{C}(11)$ & $4456(6)$ & 4957(3) & $2512(3)$ & $45(2)$ \\
\hline$C(12)$ & $4338(6)$ & $5703(3)$ & $2370(3)$ & $42(2)$ \\
\hline$C(13)$ & $3735(6)$ & 6099(3) & $1722(3)$ & $42(2)$ \\
\hline $\mathrm{C}(14)$ & $3469(5)$ & 5591(3) & $1464(3)$ & $40(2)$ \\
\hline$C(15)$ & $3917(6)$ & $4888(3)$ & 1962(3) & $45(2)$ \\
\hline$C(16)$ & $5150(7)$ & $4357(4)$ & $3145(4)$ & $79(2)$ \\
\hline $\mathrm{C}(17)$ & 4929(7) & $6040(5)$ & $2773(4)$ & $75(2)$ \\
\hline$C(18)$ & $3570(7)$ & 6919(3) & $1322(4)$ & $71(2)$ \\
\hline$C(19)$ & $2949(7)$ & $5798(4)$ & $741(3)$ & $72(2)$ \\
\hline $\mathrm{C}(20)$ & $3908(8)$ & 4193(4) & $1867(4)$ & $86(3)$ \\
\hline$C(21)$ & $1503(5)$ & $3380(3)$ & $3324(3)$ & $37(1)$ \\
\hline $\mathrm{C}(22)$ & $2589(5)$ & 2791(3) & $3592(3)$ & $34(1)$ \\
\hline$C(23)$ & $2867(6)$ & $2247(3)$ & $3342(3)$ & $41(2)$ \\
\hline$C(24)$ & $3916(6)$ & $1757(3)$ & $3541(3)$ & $46(2)$ \\
\hline
\end{tabular}




\begin{tabular}{|c|c|c|c|c|}
\hline$C(25)$ & $4722(6)$ & $1780(3)$ & $4006(3)$ & $50(2)$ \\
\hline$C(26)$ & $4453(7)$ & 2309(4) & $4263(4)$ & $55(2)$ \\
\hline$C(27)$ & $3412(6)$ & 2803(3) & 4061(3) & $46(2)$ \\
\hline $\mathrm{C}(28)$ & $264(5)$ & $3140(3)$ & $3254(3)$ & $37(1)$ \\
\hline$C(29)$ & $-114(6)$ & 2448(3) & $3702(4)$ & $49(2)$ \\
\hline$C(30)$ & $-1309(7)$ & $2262(4)$ & $3634(4)$ & $59(2)$ \\
\hline $\mathrm{C}(31)$ & $-2087(7)$ & $2755(5)$ & $3132(4)$ & $66(2)$ \\
\hline$C(32)$ & $-1722(7)$ & $3449(4)$ & $2679(4)$ & $63(2)$ \\
\hline$C(33)$ & $-558(6)$ & $3641(3)$ & $2740(3)$ & $47(2)$ \\
\hline$C(34)$ & $-274(6)$ & $6219(3)$ & $1348(3)$ & $39(2)$ \\
\hline$C(35)$ & $-324(6)$ & $7042(3)$ & $914(3)$ & $43(2)$ \\
\hline$C(36)$ & $309(6)$ & $7452(4)$ & $1141(4)$ & $54(2)$ \\
\hline$C(37)$ & $340(7)$ & 8198(4) & $736(4)$ & $65(2)$ \\
\hline$C(38)$ & $-249(8)$ & $8545(4)$ & $104(4)$ & $72(2)$ \\
\hline C(39) & $-886(7)$ & 8144(4) & $-127(4)$ & $71(2)$ \\
\hline $\mathrm{C}(40)$ & $-922(6)$ & 7399(4) & $275(3)$ & $55(2)$ \\
\hline$C(41)$ & $-1146(6)$ & $5827(3)$ & 1133(3) & $44(2)$ \\
\hline $\mathrm{C}(42)$ & $-2433(6)$ & 5990(4) & $1141(4)$ & $65(2)$ \\
\hline$C(43)$ & $-3209(7)$ & $5630(5)$ & $939(4)$ & $79(3)$ \\
\hline$C(44)$ & $-2688(8)$ & $5099(5)$ & $711(4)$ & $76(2)$ \\
\hline$C(45)$ & $-1402(8)$ & 4933(4) & 704(4) & $77(2)$ \\
\hline$C(46)$ & $-628(7)$ & 5291(4) & $918(4)$ & $62(2)$ \\
\hline$C(47)$ & $14599(6)$ & 8981(3) & $1686(3)$ & $37(1)$ \\
\hline $\mathrm{C}(48)$ & $15905(6)$ & $8942(3)$ & $1448(3)$ & $43(2)$ \\
\hline$C(49)$ & $16188(6)$ & $9655(3)$ & $948(3)$ & $43(2)$ \\
\hline$C(50)$ & $15067(6)$ & $10135(3)$ & $884(3)$ & $44(2)$ \\
\hline $\mathrm{C}(51)$ & 14091(6) & $9718(3)$ & $1350(3)$ & $39(2)$ \\
\hline$C(52)$ & $13813(7)$ & $8359(3)$ & $2126(4)$ & $60(2)$ \\
\hline$C(53)$ & $16756(7)$ & $8237(4)$ & $1621(4)$ & $65(2)$ \\
\hline$C(54)$ & $17445(7)$ & $9856(4)$ & $516(4)$ & $72(2)$ \\
\hline$C(55)$ & $14901(7)$ & $10927(3)$ & $333(3)$ & $62(2)$ \\
\hline$C(56)$ & $12733(6)$ & 9992(4) & $1452(4)$ & $58(2)$ \\
\hline$C(57)$ & $17697(5)$ & 8934(3) & $3336(3)$ & $39(2)$ \\
\hline$C(58)$ & $18422(6)$ & $9223(3)$ & 2668(3) & $39(2)$ \\
\hline$C(59)$ & $18339(5)$ & 9995(3) & 2412(3) & $36(1)$ \\
\hline $\mathrm{C}(60)$ & $17559(5)$ & 10184(3) & 2920(3) & $36(1)$ \\
\hline
\end{tabular}




\begin{tabular}{|c|c|c|c|c|}
\hline$C(61)$ & $17154(6)$ & 9533(3) & $3492(3)$ & $41(2)$ \\
\hline$C(62)$ & $17643(7)$ & $8135(3)$ & $3848(4)$ & $64(2)$ \\
\hline$C(63)$ & 19306(6) & 8783(4) & $2338(4)$ & $57(2)$ \\
\hline$C(64)$ & 19089(6) & $10498(3)$ & 1753(3) & $53(2)$ \\
\hline$C(65)$ & $17331(6)$ & 10943(3) & 2901(4) & $56(2)$ \\
\hline$C(66)$ & $16414(6)$ & 9479(4) & 4181(3) & $61(2)$ \\
\hline$C(67)$ & $14657(5)$ & $11555(3)$ & $1708(3)$ & $34(1)$ \\
\hline$C(68)$ & $13284(6)$ & $11834(3)$ & $1754(3)$ & $38(1)$ \\
\hline$C(69)$ & $12408(6)$ & $11365(4)$ & $2225(3)$ & $50(2)$ \\
\hline $\mathrm{C}(70)$ & $11141(6)$ & $11599(4)$ & $2286(4)$ & $56(2)$ \\
\hline$C(71)$ & $10708(7)$ & $12308(5)$ & $1869(4)$ & $67(2)$ \\
\hline$C(72)$ & $11547(7)$ & $12791(4)$ & $1386(4)$ & $67(2)$ \\
\hline$C(73)$ & $12826(6)$ & $12546(4)$ & 1331(3) & $49(2)$ \\
\hline$C(74)$ & $15643(5)$ & $12104(3)$ & $1402(3)$ & $35(1)$ \\
\hline$C(75)$ & $15621(6)$ & $12662(3)$ & $1628(3)$ & $45(2)$ \\
\hline$C(76)$ & $16632(7)$ & $13092(3)$ & $1417(4)$ & $56(2)$ \\
\hline$C(77)$ & $17642(7)$ & $12986(4)$ & $976(4)$ & $62(2)$ \\
\hline$C(78)$ & $17663(6)$ & $12443(3)$ & $746(4)$ & $53(2)$ \\
\hline$C(79)$ & $16680(6)$ & $12012(3)$ & $960(3)$ & $46(2)$ \\
\hline$C(80)$ & $13672(6)$ & $8707(3)$ & $3702(3)$ & $42(2)$ \\
\hline $\mathrm{C}(81)$ & $13744(6)$ & 7892(3) & $4146(3)$ & $45(2)$ \\
\hline$C(82)$ & $14685(7)$ & $7447(4)$ & $3957(4)$ & $58(2)$ \\
\hline$C(83)$ & $14831(8)$ & 6707(4) & $4375(4)$ & $72(2)$ \\
\hline$C(84)$ & $14047(10)$ & $6386(5)$ & 4992(4) & $82(3)$ \\
\hline$C(85)$ & $13121(10)$ & $6818(5)$ & $5184(4)$ & $86(3)$ \\
\hline$C(86)$ & $12954(7)$ & $7556(4)$ & $4765(3)$ & $61(2)$ \\
\hline$C(87)$ & $12525(6)$ & $9158(4)$ & $3872(3)$ & $47(2)$ \\
\hline $\mathrm{C}(88)$ & $12688(7)$ & 9724(4) & $4051(4)$ & $64(2)$ \\
\hline$C(89)$ & $11676(8)$ & $10147(5)$ & $4184(4)$ & $79(2)$ \\
\hline $\mathrm{C}(90)$ & $10454(8)$ & $10022(5)$ & $4122(4)$ & $85(3)$ \\
\hline $\mathrm{C}(91)$ & $10270(7)$ & $9477(6)$ & $3938(4)$ & $84(3)$ \\
\hline $\mathrm{C}(92)$ & $11304(7)$ & $9037(5)$ & $3821(4)$ & $72(2)$ \\
\hline
\end{tabular}


Table S13. Bond lengths $[\AA]$ and angles $\left[^{\circ}\right]$ for $\left(\mathrm{C}_{5} \mathrm{Me}_{5}\right)_{2} \mathrm{Th}\left(-\mathrm{N}=\mathrm{CPh}_{2}\right)_{2}$ (10a).

\begin{tabular}{|c|c|}
\hline $\operatorname{Th}(1)-\mathrm{N}(1)$ & $2.259(4)$ \\
\hline $\operatorname{Th}(1)-\mathrm{N}(2)$ & $2.265(5)$ \\
\hline $\operatorname{Th}(1)-\mathrm{C}(2)$ & $2.785(6)$ \\
\hline $\operatorname{Th}(1)-\mathrm{C}(15)$ & $2.796(6)$ \\
\hline $\operatorname{Th}(1)-\mathrm{C}(1)$ & $2.796(5)$ \\
\hline $\operatorname{Th}(1)-\mathrm{C}(11)$ & $2.818(6)$ \\
\hline $\operatorname{Th}(1)-\mathrm{C}(3)$ & $2.821(6)$ \\
\hline $\operatorname{Th}(1)-\mathrm{C}(4)$ & $2.829(6)$ \\
\hline $\operatorname{Th}(1)-C(5)$ & $2.829(6)$ \\
\hline $\operatorname{Th}(1)-\mathrm{C}(14)$ & $2.841(5)$ \\
\hline $\operatorname{Th}(1)-\mathrm{C}(12)$ & $2.862(6)$ \\
\hline $\operatorname{Th}(1)-\mathrm{C}(13)$ & $2.880(5)$ \\
\hline $\operatorname{Th}(2)-\mathrm{N}(4)$ & $2.255(5)$ \\
\hline $\operatorname{Th}(2)-\mathrm{N}(3)$ & $2.257(5)$ \\
\hline $\operatorname{Th}(2)-\mathrm{C}(48)$ & $2.791(5)$ \\
\hline $\operatorname{Th}(2)-\mathrm{C}(49)$ & $2.797(5)$ \\
\hline $\operatorname{Th}(2)-\mathrm{C}(60)$ & $2.835(5)$ \\
\hline $\operatorname{Th}(2)-\mathrm{C}(61)$ & $2.837(6)$ \\
\hline $\operatorname{Th}(2)-\mathrm{C}(50)$ & $2.841(6)$ \\
\hline $\operatorname{Th}(2)-\mathrm{C}(47)$ & $2.842(5)$ \\
\hline $\operatorname{Th}(2)-C(59)$ & $2.849(5)$ \\
\hline $\operatorname{Th}(2)-C(51)$ & $2.850(5)$ \\
\hline $\operatorname{Th}(2)-C(58)$ & $2.863(6)$ \\
\hline $\operatorname{Th}(2)-\mathrm{C}(57)$ & $2.863(6)$ \\
\hline $\mathrm{N}(1)-\mathrm{C}(21)$ & $1.259(7)$ \\
\hline $\mathrm{N}(2)-\mathrm{C}(34)$ & $1.272(7)$ \\
\hline N(3)-C(67) & $1.268(7)$ \\
\hline $\mathrm{N}(4)-\mathrm{C}(80)$ & $1.270(7)$ \\
\hline$C(1)-C(5)$ & $1.414(8)$ \\
\hline$C(1)-C(2)$ & $1.435(9)$ \\
\hline$C(1)-C(6)$ & $1.514(8)$ \\
\hline$C(2)-C(3)$ & $1.425(9)$ \\
\hline$C(2)-C(7)$ & $1.484(9)$ \\
\hline$C(3)-C(4)$ & $1.405(9)$ \\
\hline
\end{tabular}




\begin{tabular}{|c|c|}
\hline$C(3)-C(8)$ & $1.512(8)$ \\
\hline$C(4)-C(5)$ & $1.397(8)$ \\
\hline$C(4)-C(9)$ & $1.521(8)$ \\
\hline$C(5)-C(10)$ & $1.500(8)$ \\
\hline$C(11)-C(15)$ & $1.396(9)$ \\
\hline $\mathrm{C}(11)-\mathrm{C}(12)$ & $1.408(8)$ \\
\hline$C(11)-C(16)$ & $1.533(9)$ \\
\hline$C(12)-C(13)$ & $1.405(8)$ \\
\hline$C(12)-C(17)$ & $1.514(8)$ \\
\hline$C(13)-C(14)$ & $1.423(8)$ \\
\hline $\mathrm{C}(13)-\mathrm{C}(18)$ & $1.503(8)$ \\
\hline$C(14)-C(15)$ & $1.411(8)$ \\
\hline$C(14)-C(19)$ & $1.513(8)$ \\
\hline$C(15)-C(20)$ & $1.511(8)$ \\
\hline $\mathrm{C}(21)-\mathrm{C}(28)$ & $1.509(8)$ \\
\hline$C(21)-C(22)$ & $1.513(8)$ \\
\hline$C(22)-C(27)$ & $1.389(8)$ \\
\hline$C(22)-C(23)$ & $1.396(7)$ \\
\hline$C(23)-C(24)$ & $1.367(8)$ \\
\hline$C(24)-C(25)$ & $1.380(8)$ \\
\hline$C(25)-C(26)$ & $1.378(9)$ \\
\hline$C(26)-C(27)$ & $1.365(9)$ \\
\hline$C(28)-C(29)$ & $1.390(8)$ \\
\hline$C(28)-C(33)$ & $1.405(8)$ \\
\hline$C(29)-C(30)$ & $1.413(8)$ \\
\hline $\mathrm{C}(30)-\mathrm{C}(31)$ & $1.358(10)$ \\
\hline $\mathrm{C}(31)-\mathrm{C}(32)$ & $1.389(9)$ \\
\hline $\mathrm{C}(32)-\mathrm{C}(33)$ & $1.383(8)$ \\
\hline$C(34)-C(41)$ & $1.517(8)$ \\
\hline$C(34)-C(35)$ & $1.516(8)$ \\
\hline$C(35)-C(40)$ & $1.382(9)$ \\
\hline$C(35)-C(36)$ & $1.392(8)$ \\
\hline$C(36)-C(37)$ & $1.382(9)$ \\
\hline $\mathrm{C}(37)-\mathrm{C}(38)$ & $1.366(10)$ \\
\hline $\mathrm{C}(38)-\mathrm{C}(39)$ & $1.385(11)$ \\
\hline C(39)-C(40) & $1.380(9)$ \\
\hline
\end{tabular}




\begin{tabular}{|c|c|}
\hline$C(41)-C(42)$ & $1.365(9)$ \\
\hline$C(41)-C(46)$ & $1.375(9)$ \\
\hline$C(42)-C(43)$ & $1.379(10)$ \\
\hline$C(43)-C(44)$ & $1.384(11)$ \\
\hline $\mathrm{C}(44)-\mathrm{C}(45)$ & $1.365(10)$ \\
\hline$C(45)-C(46)$ & $1.389(9)$ \\
\hline $\mathrm{C}(47)-\mathrm{C}(51)$ & $1.413(8)$ \\
\hline $\mathrm{C}(47)-\mathrm{C}(48)$ & $1.419(8)$ \\
\hline$C(47)-C(52)$ & $1.492(8)$ \\
\hline $\mathrm{C}(48)-\mathrm{C}(49)$ & $1.415(8)$ \\
\hline $\mathrm{C}(48)-\mathrm{C}(53)$ & $1.516(8)$ \\
\hline $\mathrm{C}(49)-\mathrm{C}(50)$ & $1.418(8)$ \\
\hline$C(49)-C(54)$ & $1.511(8)$ \\
\hline$C(50)-C(51)$ & $1.415(8)$ \\
\hline $\mathrm{C}(50)-\mathrm{C}(55)$ & $1.518(8)$ \\
\hline$C(51)-C(56)$ & $1.501(8)$ \\
\hline $\mathrm{C}(57)-\mathrm{C}(58)$ & $1.409(8)$ \\
\hline $\mathrm{C}(57)-\mathrm{C}(61)$ & $1.425(8)$ \\
\hline$C(57)-C(62)$ & $1.510(8)$ \\
\hline $\mathrm{C}(58)-\mathrm{C}(59)$ & $1.419(8)$ \\
\hline $\mathrm{C}(58)-\mathrm{C}(63)$ & $1.516(8)$ \\
\hline $\mathrm{C}(59)-\mathrm{C}(60)$ & $1.406(7)$ \\
\hline $\mathrm{C}(59)-\mathrm{C}(64)$ & $1.506(8)$ \\
\hline $\mathrm{C}(60)-\mathrm{C}(61)$ & $1.415(8)$ \\
\hline$C(60)-C(65)$ & $1.517(7)$ \\
\hline $\mathrm{C}(61)-\mathrm{C}(66)$ & $1.502(8)$ \\
\hline$C(67)-C(68)$ & $1.502(8)$ \\
\hline$C(67)-C(74)$ & $1.514(7)$ \\
\hline$C(68)-C(73)$ & $1.384(8)$ \\
\hline$C(68)-C(69)$ & $1.390(8)$ \\
\hline $\mathrm{C}(69)-\mathrm{C}(70)$ & $1.377(9)$ \\
\hline $\mathrm{C}(70)-\mathrm{C}(71)$ & $1.371(10)$ \\
\hline$C(71)-C(72)$ & $1.384(10)$ \\
\hline $\mathrm{C}(72)-\mathrm{C}(73)$ & $1.394(9)$ \\
\hline $\mathrm{C}(74)-\mathrm{C}(79)$ & $1.387(8)$ \\
\hline$C(74)-C(75)$ & $1.399(7)$ \\
\hline
\end{tabular}




\begin{tabular}{|c|c|}
\hline$C(75)-C(76)$ & $1.385(8)$ \\
\hline$C(76)-C(77)$ & $1.376(9)$ \\
\hline $\mathrm{C}(77)-\mathrm{C}(78)$ & $1.377(9)$ \\
\hline $\mathrm{C}(78)-\mathrm{C}(79)$ & $1.362(8)$ \\
\hline $\mathrm{C}(80)-\mathrm{C}(81)$ & $1.504(8)$ \\
\hline $\mathrm{C}(80)-\mathrm{C}(87)$ & $1.527(9)$ \\
\hline $\mathrm{C}(81)-\mathrm{C}(86)$ & $1.389(8)$ \\
\hline $\mathrm{C}(81)-\mathrm{C}(82)$ & $1.393(9)$ \\
\hline $\mathrm{C}(82)-\mathrm{C}(83)$ & $1.371(9)$ \\
\hline $\mathrm{C}(83)-\mathrm{C}(84)$ & $1.378(11)$ \\
\hline $\mathrm{C}(84)-\mathrm{C}(85)$ & $1.369(12)$ \\
\hline $\mathrm{C}(85)-\mathrm{C}(86)$ & $1.369(10)$ \\
\hline $\mathrm{C}(87)-\mathrm{C}(92)$ & $1.379(9)$ \\
\hline $\mathrm{C}(87)-\mathrm{C}(88)$ & $1.389(9)$ \\
\hline $\mathrm{C}(88)-\mathrm{C}(89)$ & $1.365(10)$ \\
\hline $\mathrm{C}(89)-\mathrm{C}(90)$ & $1.390(10)$ \\
\hline $\mathrm{C}(90)-\mathrm{C}(91)$ & $1.361(11)$ \\
\hline $\mathrm{C}(91)-\mathrm{C}(92)$ & $1.389(11)$ \\
\hline $\mathrm{N}(1)-\mathrm{Th}(1)-\mathrm{N}(2)$ & $108.95(17)$ \\
\hline $\mathrm{N}(1)-\mathrm{Th}(1)-\mathrm{C}(2)$ & $96.45(18)$ \\
\hline $\mathrm{N}(2)-\mathrm{Th}(1)-\mathrm{C}(2)$ & $125.14(18)$ \\
\hline $\mathrm{N}(1)-\operatorname{Th}(1)-\mathrm{C}(15)$ & $80.73(17)$ \\
\hline $\mathrm{N}(2)-\operatorname{Th}(1)-\mathrm{C}(15)$ & $100.66(19)$ \\
\hline$C(2)-\operatorname{Th}(1)-C(15)$ & $131.6(2)$ \\
\hline $\mathrm{N}(1)-\operatorname{Th}(1)-\mathrm{C}(1)$ & $122.30(17)$ \\
\hline $\mathrm{N}(2)-\operatorname{Th}(1)-\mathrm{C}(1)$ & $99.28(18)$ \\
\hline $\mathrm{C}(2)-\operatorname{Th}(1)-\mathrm{C}(1)$ & $29.79(18)$ \\
\hline$C(15)-\operatorname{Th}(1)-C(1)$ & $141.84(18)$ \\
\hline $\mathrm{N}(1)-\operatorname{Th}(1)-\mathrm{C}(11)$ & $88.14(18)$ \\
\hline $\mathrm{N}(2)-\operatorname{Th}(1)-\mathrm{C}(11)$ & $124.62(17)$ \\
\hline$C(2)-\operatorname{Th}(1)-C(11)$ & $103.3(2)$ \\
\hline $\mathrm{C}(15)-\mathrm{Th}(1)-\mathrm{C}(11)$ & $28.80(18)$ \\
\hline $\mathrm{C}(1)-\operatorname{Th}(1)-\mathrm{C}(11)$ & $115.35(19)$ \\
\hline $\mathrm{N}(1)-\mathrm{Th}(1)-\mathrm{C}(3)$ & $74.19(16)$ \\
\hline $\mathrm{N}(2)-\mathrm{Th}(1)-\mathrm{C}(3)$ & $114.7(2)$ \\
\hline
\end{tabular}




\begin{tabular}{|c|c|}
\hline $\mathrm{C}(2)-\mathrm{Th}(1)-\mathrm{C}(3)$ & $29.44(18)$ \\
\hline $\mathrm{C}(15)-\mathrm{Th}(1)-\mathrm{C}(3)$ & $141.6(2)$ \\
\hline $\mathrm{C}(1)-\mathrm{Th}(1)-\mathrm{C}(3)$ & $48.23(17)$ \\
\hline $\mathrm{C}(11)-\mathrm{Th}(1)-\mathrm{C}(3)$ & $120.7(2)$ \\
\hline $\mathrm{N}(1)-\mathrm{Th}(1)-\mathrm{C}(4)$ & $84.59(17)$ \\
\hline $\mathrm{N}(2)-\mathrm{Th}(1)-\mathrm{C}(4)$ & $85.92(19)$ \\
\hline $\mathrm{C}(2)-\mathrm{Th}(1)-\mathrm{C}(4)$ & $48.24(19)$ \\
\hline $\mathrm{C}(15)-\mathrm{Th}(1)-\mathrm{C}(4)$ & $165.19(17)$ \\
\hline $\mathrm{C}(1)-\mathrm{Th}(1)-\mathrm{C}(4)$ & $47.84(16)$ \\
\hline $\mathrm{C}(11)-\mathrm{Th}(1)-\mathrm{C}(4)$ & 149.22(19) \\
\hline $\mathrm{C}(3)-\mathrm{Th}(1)-\mathrm{C}(4)$ & $28.80(18)$ \\
\hline $\mathrm{N}(1)-\operatorname{Th}(1)-\mathrm{C}(5)$ & $113.17(17)$ \\
\hline $\mathrm{N}(2)-\mathrm{Th}(1)-\mathrm{C}(5)$ & $76.81(17)$ \\
\hline $\mathrm{C}(2)-\mathrm{Th}(1)-\mathrm{C}(5)$ & $48.39(19)$ \\
\hline $\mathrm{C}(15)-\mathrm{Th}(1)-\mathrm{C}(5)$ & $166.02(16)$ \\
\hline $\mathrm{C}(1)-\mathrm{Th}(1)-\mathrm{C}(5)$ & $29.12(17)$ \\
\hline $\mathrm{C}(11)-\mathrm{Th}(1)-\mathrm{C}(5)$ & $144.33(18)$ \\
\hline $\mathrm{C}(3)-\mathrm{Th}(1)-\mathrm{C}(5)$ & $47.52(18)$ \\
\hline $\mathrm{C}(4)-\mathrm{Th}(1)-\mathrm{C}(5)$ & $28.58(16)$ \\
\hline $\mathrm{N}(1)-\operatorname{Th}(1)-\mathrm{C}(14)$ & $104.23(17)$ \\
\hline $\mathrm{N}(2)-\mathrm{Th}(1)-\mathrm{C}(14)$ & $77.17(17)$ \\
\hline $\mathrm{C}(2)-\mathrm{Th}(1)-\mathrm{C}(14)$ & $142.35(18)$ \\
\hline $\mathrm{C}(15)-\mathrm{Th}(1)-\mathrm{C}(14)$ & $28.98(17)$ \\
\hline $\mathrm{C}(1)-\mathrm{Th}(1)-\mathrm{C}(14)$ & $131.06(17)$ \\
\hline $\mathrm{C}(11)-\mathrm{Th}(1)-\mathrm{C}(14)$ & $47.45(18)$ \\
\hline $\mathrm{C}(3)-\mathrm{Th}(1)-\mathrm{C}(14)$ & $168.1(2)$ \\
\hline $\mathrm{C}(4)-\mathrm{Th}(1)-\mathrm{C}(14)$ & $162.7(2)$ \\
\hline $\mathrm{C}(5)-\mathrm{Th}(1)-\mathrm{C}(14)$ & $139.62(17)$ \\
\hline $\mathrm{N}(1)-\mathrm{Th}(1)-\mathrm{C}(12)$ & $116.59(18)$ \\
\hline $\mathrm{N}(2)-\mathrm{Th}(1)-\mathrm{C}(12)$ & $113.52(18)$ \\
\hline $\mathrm{C}(2)-\mathrm{Th}(1)-\mathrm{C}(12)$ & $95.35(18)$ \\
\hline $\mathrm{C}(15)-\mathrm{Th}(1)-\mathrm{C}(12)$ & $47.38(17)$ \\
\hline $\mathrm{C}(1)-\mathrm{Th}(1)-\mathrm{C}(12)$ & $94.73(17)$ \\
\hline $\mathrm{C}(11)-\mathrm{Th}(1)-\mathrm{C}(12)$ & $28.70(17)$ \\
\hline$C(3)-T h(1)-C(12)$ & $122.41(19)$ \\
\hline $\mathrm{C}(4)-\mathrm{Th}(1)-\mathrm{C}(12)$ & $141.40(17)$ \\
\hline
\end{tabular}




\begin{tabular}{|c|c|}
\hline $\mathrm{C}(5)-\mathrm{Th}(1)-\mathrm{C}(12)$ & $120.66(17)$ \\
\hline $\mathrm{C}(14)-\mathrm{Th}(1)-\mathrm{C}(12)$ & $47.25(16)$ \\
\hline $\mathrm{N}(1)-\mathrm{Th}(1)-\mathrm{C}(13)$ & $128.14(17)$ \\
\hline $\mathrm{N}(2)-\mathrm{Th}(1)-\mathrm{C}(13)$ & $85.33(18)$ \\
\hline $\mathrm{C}(2)-\mathrm{Th}(1)-\mathrm{C}(13)$ & $115.76(19)$ \\
\hline $\mathrm{C}(15)-\mathrm{Th}(1)-\mathrm{C}(13)$ & $47.42(16)$ \\
\hline $\mathrm{C}(1)-\mathrm{Th}(1)-\mathrm{C}(13)$ & $102.79(17)$ \\
\hline $\mathrm{C}(11)-\mathrm{Th}(1)-\mathrm{C}(13)$ & $47.09(17)$ \\
\hline $\mathrm{C}(3)-\mathrm{Th}(1)-\mathrm{C}(13)$ & $145.19(18)$ \\
\hline$C(4)-\operatorname{Th}(1)-C(13)$ & $147.16(17)$ \\
\hline $\mathrm{C}(5)-\mathrm{Th}(1)-\mathrm{C}(13)$ & $118.65(16)$ \\
\hline $\mathrm{C}(14)-\mathrm{Th}(1)-\mathrm{C}(13)$ & $28.80(16)$ \\
\hline $\mathrm{C}(12)-\mathrm{Th}(1)-\mathrm{C}(13)$ & $28.33(17)$ \\
\hline $\mathrm{N}(4)-\mathrm{Th}(2)-\mathrm{N}(3)$ & $106.50(17)$ \\
\hline $\mathrm{N}(4)-\operatorname{Th}(2)-\mathrm{C}(48)$ & $97.92(17)$ \\
\hline $\mathrm{N}(3)-\mathrm{Th}(2)-\mathrm{C}(48)$ & $122.31(17)$ \\
\hline $\mathrm{N}(4)-\mathrm{Th}(2)-\mathrm{C}(49)$ & $124.05(16)$ \\
\hline $\mathrm{N}(3)-\mathrm{Th}(2)-\mathrm{C}(49)$ & $98.49(17)$ \\
\hline $\mathrm{C}(48)-\mathrm{Th}(2)-\mathrm{C}(49)$ & $29.33(16)$ \\
\hline $\mathrm{N}(4)-\operatorname{Th}(2)-\mathrm{C}(60)$ & $105.82(16)$ \\
\hline $\mathrm{N}(3)-\mathrm{Th}(2)-\mathrm{C}(60)$ & $82.01(16)$ \\
\hline $\mathrm{C}(48)-\mathrm{Th}(2)-\mathrm{C}(60)$ & $139.34(17)$ \\
\hline$C(49)-T h(2)-C(60)$ & $126.99(17)$ \\
\hline $\mathrm{N}(4)-\operatorname{Th}(2)-\mathrm{C}(61)$ & $79.48(16)$ \\
\hline $\mathrm{N}(3)-\mathrm{Th}(2)-\mathrm{C}(61)$ & $100.71(17)$ \\
\hline $\mathrm{C}(48)-\mathrm{Th}(2)-\mathrm{C}(61)$ & $135.21(18)$ \\
\hline $\mathrm{C}(49)-\mathrm{Th}(2)-\mathrm{C}(61)$ & $143.27(18)$ \\
\hline $\mathrm{C}(60)-\mathrm{Th}(2)-\mathrm{C}(61)$ & $28.89(15)$ \\
\hline $\mathrm{N}(4)-\operatorname{Th}(2)-\mathrm{C}(50)$ & $116.04(18)$ \\
\hline $\mathrm{N}(3)-\mathrm{Th}(2)-\mathrm{C}(50)$ & $74.26(16)$ \\
\hline $\mathrm{C}(48)-\mathrm{Th}(2)-\mathrm{C}(50)$ & $48.09(17)$ \\
\hline $\mathrm{C}(49)-\mathrm{Th}(2)-\mathrm{C}(50)$ & 29.11(17) \\
\hline $\mathrm{C}(60)-\mathrm{Th}(2)-\mathrm{C}(50)$ & $136.10(17)$ \\
\hline$C(61)-T h(2)-C(50)$ & $164.40(17)$ \\
\hline $\mathrm{N}(4)-\mathrm{Th}(2)-\mathrm{C}(47)$ & $76.50(16)$ \\
\hline $\mathrm{N}(3)-\operatorname{Th}(2)-\mathrm{C}(47)$ & $110.03(17)$ \\
\hline
\end{tabular}




\begin{tabular}{|c|c|}
\hline $\mathrm{C}(48)-\mathrm{Th}(2)-\mathrm{C}(47)$ & $29.16(16)$ \\
\hline $\mathrm{C}(49)-\mathrm{Th}(2)-\mathrm{C}(47)$ & $47.83(16)$ \\
\hline $\mathrm{C}(60)-\mathrm{Th}(2)-\mathrm{C}(47)$ & $166.83(16)$ \\
\hline $\mathrm{C}(61)-\mathrm{Th}(2)-\mathrm{C}(47)$ & $145.19(17)$ \\
\hline $\mathrm{C}(50)-\mathrm{Th}(2)-\mathrm{C}(47)$ & $47.48(16)$ \\
\hline $\mathrm{N}(4)-\operatorname{Th}(2)-\mathrm{C}(59)$ & $125.65(17)$ \\
\hline N(3)-Th(2)-C(59) & $95.31(16)$ \\
\hline $\mathrm{C}(48)-\mathrm{Th}(2)-\mathrm{C}(59)$ & $111.34(18)$ \\
\hline $\mathrm{C}(49)-\mathrm{Th}(2)-\mathrm{C}(59)$ & $100.01(17)$ \\
\hline$C(60)-T h(2)-C(59)$ & $28.63(15)$ \\
\hline $\mathrm{C}(61)-\mathrm{Th}(2)-\mathrm{C}(59)$ & $47.35(16)$ \\
\hline $\mathrm{C}(50)-\mathrm{Th}(2)-\mathrm{C}(59)$ & $117.66(17)$ \\
\hline $\mathrm{C}(47)-\mathrm{Th}(2)-\mathrm{C}(59)$ & $140.41(16)$ \\
\hline $\mathrm{N}(4)-\mathrm{Th}(2)-\mathrm{C}(51)$ & $87.24(17)$ \\
\hline $\mathrm{N}(3)-\operatorname{Th}(2)-\mathrm{C}(51)$ & $81.45(16)$ \\
\hline $\mathrm{C}(48)-\mathrm{Th}(2)-\mathrm{C}(51)$ & $48.00(17)$ \\
\hline $\mathrm{C}(49)-\mathrm{Th}(2)-\mathrm{C}(51)$ & $47.83(17)$ \\
\hline $\mathrm{C}(60)-\mathrm{Th}(2)-\mathrm{C}(51)$ & $161.31(17)$ \\
\hline $\mathrm{C}(61)-\mathrm{Th}(2)-\mathrm{C}(51)$ & $166.63(16)$ \\
\hline $\mathrm{C}(50)-\mathrm{Th}(2)-\mathrm{C}(51)$ & $28.80(16)$ \\
\hline $\mathrm{C}(47)-\mathrm{Th}(2)-\mathrm{C}(51)$ & $28.75(16)$ \\
\hline $\mathrm{C}(59)-\mathrm{Th}(2)-\mathrm{C}(51)$ & $145.94(16)$ \\
\hline $\mathrm{N}(4)-\mathrm{Th}(2)-\mathrm{C}(58)$ & $108.78(17)$ \\
\hline $\mathrm{N}(3)-\mathrm{Th}(2)-\mathrm{C}(58)$ & $123.99(16)$ \\
\hline $\mathrm{C}(48)-\mathrm{Th}(2)-\mathrm{C}(58)$ & $94.07(17)$ \\
\hline $\mathrm{C}(49)-\mathrm{Th}(2)-\mathrm{C}(58)$ & $96.18(18)$ \\
\hline $\mathrm{C}(60)-\mathrm{Th}(2)-\mathrm{C}(58)$ & $47.30(16)$ \\
\hline $\mathrm{C}(61)-\mathrm{Th}(2)-\mathrm{C}(58)$ & $47.26(17)$ \\
\hline $\mathrm{C}(50)-\mathrm{Th}(2)-\mathrm{C}(58)$ & $123.17(18)$ \\
\hline $\mathrm{C}(47)-\mathrm{Th}(2)-\mathrm{C}(58)$ & $119.54(16)$ \\
\hline $\mathrm{C}(59)-\mathrm{Th}(2)-\mathrm{C}(58)$ & $28.77(15)$ \\
\hline $\mathrm{C}(51)-\mathrm{Th}(2)-\mathrm{C}(58)$ & $141.30(16)$ \\
\hline $\mathrm{N}(4)-\mathrm{Th}(2)-\mathrm{C}(57)$ & $81.50(17)$ \\
\hline $\mathrm{N}(3)-\mathrm{Th}(2)-\mathrm{C}(57)$ & $128.23(16)$ \\
\hline $\mathrm{C}(48)-\mathrm{Th}(2)-\mathrm{C}(57)$ & $106.26(17)$ \\
\hline $\mathrm{C}(49)-\mathrm{Th}(2)-\mathrm{C}(57)$ & $119.37(19)$ \\
\hline
\end{tabular}




\begin{tabular}{|c|c|}
\hline $\mathrm{C}(60)-\mathrm{Th}(2)-\mathrm{C}(57)$ & $47.58(16)$ \\
\hline$C(61)-T h(2)-C(57)$ & $28.96(16)$ \\
\hline $\mathrm{C}(50)-\mathrm{Th}(2)-\mathrm{C}(57)$ & $148.41(18)$ \\
\hline$C(47)-\operatorname{Th}(2)-C(57)$ & $121.41(16)$ \\
\hline $\mathrm{C}(59)-\mathrm{Th}(2)-\mathrm{C}(57)$ & $47.34(16)$ \\
\hline $\mathrm{C}(51)-\mathrm{Th}(2)-\mathrm{C}(57)$ & $150.16(16)$ \\
\hline $\mathrm{C}(58)-\mathrm{Th}(2)-\mathrm{C}(57)$ & $28.49(16)$ \\
\hline $\mathrm{C}(21)-\mathrm{N}(1)-\mathrm{Th}(1)$ & $174.0(4)$ \\
\hline $\mathrm{C}(34)-\mathrm{N}(2)-\mathrm{Th}(1)$ & $179.4(5)$ \\
\hline $\mathrm{C}(67)-\mathrm{N}(3)-\mathrm{Th}(2)$ & $173.7(4)$ \\
\hline $\mathrm{C}(80)-\mathrm{N}(4)-\mathrm{Th}(2)$ & $171.5(5)$ \\
\hline$C(5)-C(1)-C(2)$ & $107.7(5)$ \\
\hline$C(5)-C(1)-C(6)$ & $125.9(6)$ \\
\hline $\mathrm{C}(2)-\mathrm{C}(1)-\mathrm{C}(6)$ & $126.0(6)$ \\
\hline $\mathrm{C}(5)-\mathrm{C}(1)-\mathrm{Th}(1)$ & $76.7(3)$ \\
\hline $\mathrm{C}(2)-\mathrm{C}(1)-\mathrm{Th}(1)$ & $74.7(3)$ \\
\hline $\mathrm{C}(6)-\mathrm{C}(1)-\mathrm{Th}(1)$ & $119.9(4)$ \\
\hline $\mathrm{C}(3)-\mathrm{C}(2)-\mathrm{C}(1)$ & $106.8(6)$ \\
\hline $\mathrm{C}(3)-\mathrm{C}(2)-\mathrm{C}(7)$ & $126.0(7)$ \\
\hline$C(1)-C(2)-C(7)$ & $127.0(6)$ \\
\hline $\mathrm{C}(3)-\mathrm{C}(2)-\mathrm{Th}(1)$ & $76.7(3)$ \\
\hline $\mathrm{C}(1)-\mathrm{C}(2)-\mathrm{Th}(1)$ & $75.5(3)$ \\
\hline $\mathrm{C}(7)-\mathrm{C}(2)-\mathrm{Th}(1)$ & $118.1(4)$ \\
\hline $\mathrm{C}(4)-\mathrm{C}(3)-\mathrm{C}(2)$ & $108.3(5)$ \\
\hline $\mathrm{C}(4)-\mathrm{C}(3)-\mathrm{C}(8)$ & $126.7(6)$ \\
\hline $\mathrm{C}(2)-\mathrm{C}(3)-\mathrm{C}(8)$ & $124.5(7)$ \\
\hline $\mathrm{C}(4)-\mathrm{C}(3)-\mathrm{Th}(1)$ & $75.9(3)$ \\
\hline $\mathrm{C}(2)-\mathrm{C}(3)-\mathrm{Th}(1)$ & $73.9(3)$ \\
\hline $\mathrm{C}(8)-\mathrm{C}(3)-\mathrm{Th}(1)$ & $122.4(4)$ \\
\hline $\mathrm{C}(5)-\mathrm{C}(4)-\mathrm{C}(3)$ & $108.7(5)$ \\
\hline$C(5)-C(4)-C(9)$ & $124.7(6)$ \\
\hline $\mathrm{C}(3)-\mathrm{C}(4)-\mathrm{C}(9)$ & $126.6(6)$ \\
\hline $\mathrm{C}(5)-\mathrm{C}(4)-\mathrm{Th}(1)$ & $75.7(3)$ \\
\hline $\mathrm{C}(3)-\mathrm{C}(4)-\mathrm{Th}(1)$ & $75.3(3)$ \\
\hline $\mathrm{C}(9)-\mathrm{C}(4)-\mathrm{Th}(1)$ & $117.2(4)$ \\
\hline$C(4)-C(5)-C(1)$ & $108.5(5)$ \\
\hline
\end{tabular}




\begin{tabular}{|c|c|}
\hline$C(4)-C(5)-C(10)$ & $124.8(6)$ \\
\hline$C(1)-C(5)-C(10)$ & $126.0(5)$ \\
\hline $\mathrm{C}(4)-\mathrm{C}(5)-\mathrm{Th}(1)$ & $75.7(3)$ \\
\hline $\mathrm{C}(1)-\mathrm{C}(5)-\operatorname{Th}(1)$ & $74.1(3)$ \\
\hline$C(10)-C(5)-T h(1)$ & $124.1(4)$ \\
\hline$C(15)-C(11)-C(12)$ & $108.3(5)$ \\
\hline$C(15)-C(11)-C(16)$ & $128.0(6)$ \\
\hline$C(12)-C(11)-C(16)$ & $123.6(6)$ \\
\hline $\mathrm{C}(15)-\mathrm{C}(11)-\mathrm{Th}(1)$ & $74.7(4)$ \\
\hline $\mathrm{C}(12)-\mathrm{C}(11)-\mathrm{Th}(1)$ & $77.4(3)$ \\
\hline $\mathrm{C}(16)-\mathrm{C}(11)-\mathrm{Th}(1)$ & $117.5(4)$ \\
\hline$C(13)-C(12)-C(11)$ & $108.1(5)$ \\
\hline$C(13)-C(12)-C(17)$ & $125.0(6)$ \\
\hline $\mathrm{C}(11)-\mathrm{C}(12)-\mathrm{C}(17)$ & $126.4(6)$ \\
\hline $\mathrm{C}(13)-\mathrm{C}(12)-\mathrm{Th}(1)$ & $76.6(3)$ \\
\hline $\mathrm{C}(11)-\mathrm{C}(12)-\mathrm{Th}(1)$ & $73.9(3)$ \\
\hline $\mathrm{C}(17)-\mathrm{C}(12)-\mathrm{Th}(1)$ & $122.6(4)$ \\
\hline$C(12)-C(13)-C(14)$ & $107.8(5)$ \\
\hline $\mathrm{C}(12)-\mathrm{C}(13)-\mathrm{C}(18)$ & $125.7(6)$ \\
\hline $\mathrm{C}(14)-\mathrm{C}(13)-\mathrm{C}(18)$ & $126.0(6)$ \\
\hline $\mathrm{C}(12)-\mathrm{C}(13)-\mathrm{Th}(1)$ & $75.1(3)$ \\
\hline $\mathrm{C}(14)-\mathrm{C}(13)-\mathrm{Th}(1)$ & $74.1(3)$ \\
\hline $\mathrm{C}(18)-\mathrm{C}(13)-\mathrm{Th}(1)$ & $123.2(4)$ \\
\hline$C(15)-C(14)-C(13)$ & $107.4(5)$ \\
\hline$C(15)-C(14)-C(19)$ & $127.5(6)$ \\
\hline$C(13)-C(14)-C(19)$ & $124.8(6)$ \\
\hline $\mathrm{C}(15)-\mathrm{C}(14)-\mathrm{Th}(1)$ & $73.7(3)$ \\
\hline $\mathrm{C}(13)-\mathrm{C}(14)-\mathrm{Th}(1)$ & $77.1(3)$ \\
\hline C(19)-C(14)-Th(1) & $120.7(4)$ \\
\hline$C(11)-C(15)-C(14)$ & $108.4(5)$ \\
\hline $\mathrm{C}(11)-\mathrm{C}(15)-\mathrm{C}(20)$ & $126.6(6)$ \\
\hline$C(14)-C(15)-C(20)$ & $124.8(6)$ \\
\hline $\mathrm{C}(11)-\mathrm{C}(15)-\mathrm{Th}(1)$ & $76.5(3)$ \\
\hline$C(14)-C(15)-T h(1)$ & $77.3(3)$ \\
\hline$C(20)-C(15)-T h(1)$ & $116.8(4)$ \\
\hline $\mathrm{N}(1)-\mathrm{C}(21)-\mathrm{C}(28)$ & $121.6(5)$ \\
\hline
\end{tabular}




\begin{tabular}{|c|c|}
\hline $\mathrm{N}(1)-\mathrm{C}(21)-\mathrm{C}(22)$ & $121.5(5)$ \\
\hline $\mathrm{C}(28)-\mathrm{C}(21)-\mathrm{C}(22)$ & $116.9(5)$ \\
\hline $\mathrm{C}(27)-\mathrm{C}(22)-\mathrm{C}(23)$ & $117.1(5)$ \\
\hline $\mathrm{C}(27)-\mathrm{C}(22)-\mathrm{C}(21)$ & $120.8(5)$ \\
\hline$C(23)-C(22)-C(21)$ & $122.0(5)$ \\
\hline$C(24)-C(23)-C(22)$ & $121.3(6)$ \\
\hline $\mathrm{C}(23)-\mathrm{C}(24)-\mathrm{C}(25)$ & $120.6(6)$ \\
\hline$C(26)-C(25)-C(24)$ & $118.7(6)$ \\
\hline$C(27)-C(26)-C(25)$ & $120.7(6)$ \\
\hline$C(26)-C(27)-C(22)$ & $121.6(6)$ \\
\hline $\mathrm{C}(29)-\mathrm{C}(28)-\mathrm{C}(33)$ & $118.8(5)$ \\
\hline$C(29)-C(28)-C(21)$ & $122.0(5)$ \\
\hline $\mathrm{C}(33)-\mathrm{C}(28)-\mathrm{C}(21)$ & 119.1(5) \\
\hline $\mathrm{C}(28)-\mathrm{C}(29)-\mathrm{C}(30)$ & $119.7(6)$ \\
\hline$C(31)-C(30)-C(29)$ & $120.5(6)$ \\
\hline $\mathrm{C}(30)-\mathrm{C}(31)-\mathrm{C}(32)$ & $120.6(6)$ \\
\hline $\mathrm{C}(33)-\mathrm{C}(32)-\mathrm{C}(31)$ & $119.8(7)$ \\
\hline $\mathrm{C}(32)-\mathrm{C}(33)-\mathrm{C}(28)$ & $120.7(6)$ \\
\hline $\mathrm{N}(2)-\mathrm{C}(34)-\mathrm{C}(41)$ & $121.9(5)$ \\
\hline $\mathrm{N}(2)-\mathrm{C}(34)-\mathrm{C}(35)$ & $122.2(6)$ \\
\hline $\mathrm{C}(41)-\mathrm{C}(34)-\mathrm{C}(35)$ & $115.9(5)$ \\
\hline$C(40)-C(35)-C(36)$ & $118.4(6)$ \\
\hline $\mathrm{C}(40)-\mathrm{C}(35)-\mathrm{C}(34)$ & $122.2(6)$ \\
\hline$C(36)-C(35)-C(34)$ & $119.3(6)$ \\
\hline$C(37)-C(36)-C(35)$ & $120.7(7)$ \\
\hline $\mathrm{C}(38)-\mathrm{C}(37)-\mathrm{C}(36)$ & $120.5(7)$ \\
\hline $\mathrm{C}(37)-\mathrm{C}(38)-\mathrm{C}(39)$ & $119.3(7)$ \\
\hline $\mathrm{C}(40)-\mathrm{C}(39)-\mathrm{C}(38)$ & $120.6(8)$ \\
\hline$C(39)-C(40)-C(35)$ & $120.5(7)$ \\
\hline$C(42)-C(41)-C(46)$ & $118.5(6)$ \\
\hline $\mathrm{C}(42)-\mathrm{C}(41)-\mathrm{C}(34)$ & $122.3(6)$ \\
\hline $\mathrm{C}(46)-\mathrm{C}(41)-\mathrm{C}(34)$ & 119.2(6) \\
\hline$C(41)-C(42)-C(43)$ & $121.4(7)$ \\
\hline$C(42)-C(43)-C(44)$ & $120.2(8)$ \\
\hline$C(45)-C(44)-C(43)$ & $118.6(7)$ \\
\hline$C(44)-C(45)-C(46)$ & $120.9(8)$ \\
\hline
\end{tabular}




\begin{tabular}{|c|c|}
\hline$C(41)-C(46)-C(45)$ & $120.4(7)$ \\
\hline $\mathrm{C}(51)-\mathrm{C}(47)-\mathrm{C}(48)$ & $108.3(5)$ \\
\hline $\mathrm{C}(51)-\mathrm{C}(47)-\mathrm{C}(52)$ & $123.7(6)$ \\
\hline$C(48)-C(47)-C(52)$ & $127.5(6)$ \\
\hline $\mathrm{C}(51)-\mathrm{C}(47)-\mathrm{Th}(2)$ & $75.9(3)$ \\
\hline $\mathrm{C}(48)-\mathrm{C}(47)-\mathrm{Th}(2)$ & $73.4(3)$ \\
\hline $\mathrm{C}(52)-\mathrm{C}(47)-\mathrm{Th}(2)$ & $123.4(4)$ \\
\hline$C(49)-C(48)-C(47)$ & $107.6(5)$ \\
\hline $\mathrm{C}(49)-\mathrm{C}(48)-\mathrm{C}(53)$ & $127.2(6)$ \\
\hline $\mathrm{C}(47)-\mathrm{C}(48)-\mathrm{C}(53)$ & $124.6(6)$ \\
\hline $\mathrm{C}(49)-\mathrm{C}(48)-\mathrm{Th}(2)$ & $75.6(3)$ \\
\hline $\mathrm{C}(47)-\mathrm{C}(48)-\mathrm{Th}(2)$ & $77.4(3)$ \\
\hline $\mathrm{C}(53)-\mathrm{C}(48)-\mathrm{Th}(2)$ & $120.2(4)$ \\
\hline $\mathrm{C}(48)-\mathrm{C}(49)-\mathrm{C}(50)$ & $108.3(5)$ \\
\hline $\mathrm{C}(48)-\mathrm{C}(49)-\mathrm{C}(54)$ & $125.5(6)$ \\
\hline $\mathrm{C}(50)-\mathrm{C}(49)-\mathrm{C}(54)$ & $126.1(6)$ \\
\hline $\mathrm{C}(48)-\mathrm{C}(49)-\mathrm{Th}(2)$ & $75.1(3)$ \\
\hline $\mathrm{C}(50)-\mathrm{C}(49)-\mathrm{Th}(2)$ & $77.2(3)$ \\
\hline $\mathrm{C}(54)-\mathrm{C}(49)-\mathrm{Th}(2)$ & $117.5(4)$ \\
\hline $\mathrm{C}(51)-\mathrm{C}(50)-\mathrm{C}(49)$ & $107.8(5)$ \\
\hline $\mathrm{C}(51)-\mathrm{C}(50)-\mathrm{C}(55)$ & $126.0(6)$ \\
\hline$C(49)-C(50)-C(55)$ & $125.6(6)$ \\
\hline $\mathrm{C}(51)-\mathrm{C}(50)-\mathrm{Th}(2)$ & $75.9(3)$ \\
\hline $\mathrm{C}(49)-\mathrm{C}(50)-\mathrm{Th}(2)$ & $73.7(3)$ \\
\hline $\mathrm{C}(55)-\mathrm{C}(50)-\mathrm{Th}(2)$ & $123.1(4)$ \\
\hline$C(47)-C(51)-C(50)$ & $108.0(5)$ \\
\hline $\mathrm{C}(47)-\mathrm{C}(51)-\mathrm{C}(56)$ & $125.2(6)$ \\
\hline $\mathrm{C}(50)-\mathrm{C}(51)-\mathrm{C}(56)$ & $126.8(6)$ \\
\hline $\mathrm{C}(47)-\mathrm{C}(51)-\mathrm{Th}(2)$ & $75.3(3)$ \\
\hline $\mathrm{C}(50)-\mathrm{C}(51)-\mathrm{Th}(2)$ & $75.3(3)$ \\
\hline $\mathrm{C}(56)-\mathrm{C}(51)-\mathrm{Th}(2)$ & $117.8(4)$ \\
\hline $\mathrm{C}(58)-\mathrm{C}(57)-\mathrm{C}(61)$ & $107.4(5)$ \\
\hline $\mathrm{C}(58)-\mathrm{C}(57)-\mathrm{C}(62)$ & $126.7(6)$ \\
\hline$C(61)-C(57)-C(62)$ & $125.4(6)$ \\
\hline $\mathrm{C}(58)-\mathrm{C}(57)-\mathrm{Th}(2)$ & $75.7(3)$ \\
\hline $\mathrm{C}(61)-\mathrm{C}(57)-\mathrm{Th}(2)$ & $74.5(3)$ \\
\hline
\end{tabular}




\begin{tabular}{|c|c|}
\hline $\mathrm{C}(62)-\mathrm{C}(57)-\mathrm{Th}(2)$ & $122.0(4)$ \\
\hline $\mathrm{C}(57)-\mathrm{C}(58)-\mathrm{C}(59)$ & $108.4(5)$ \\
\hline $\mathrm{C}(57)-\mathrm{C}(58)-\mathrm{C}(63)$ & $125.8(5)$ \\
\hline $\mathrm{C}(59)-\mathrm{C}(58)-\mathrm{C}(63)$ & $125.2(5)$ \\
\hline $\mathrm{C}(57)-\mathrm{C}(58)-\mathrm{Th}(2)$ & $75.8(3)$ \\
\hline $\mathrm{C}(59)-\mathrm{C}(58)-\mathrm{Th}(2)$ & $75.1(3)$ \\
\hline $\mathrm{C}(63)-\mathrm{C}(58)-\mathrm{Th}(2)$ & $122.6(4)$ \\
\hline$C(60)-C(59)-C(58)$ & $108.0(5)$ \\
\hline $\mathrm{C}(60)-\mathrm{C}(59)-\mathrm{C}(64)$ & $127.1(5)$ \\
\hline$C(58)-C(59)-C(64)$ & $124.6(5)$ \\
\hline $\mathrm{C}(60)-\mathrm{C}(59)-\mathrm{Th}(2)$ & $75.1(3)$ \\
\hline $\mathrm{C}(58)-\mathrm{C}(59)-\mathrm{Th}(2)$ & $76.1(3)$ \\
\hline $\mathrm{C}(64)-\mathrm{C}(59)-\mathrm{Th}(2)$ & $120.3(4)$ \\
\hline $\mathrm{C}(59)-\mathrm{C}(60)-\mathrm{C}(61)$ & $108.1(5)$ \\
\hline $\mathrm{C}(59)-\mathrm{C}(60)-\mathrm{C}(65)$ & $125.5(5)$ \\
\hline $\mathrm{C}(61)-\mathrm{C}(60)-\mathrm{C}(65)$ & $126.0(5)$ \\
\hline $\mathrm{C}(59)-\mathrm{C}(60)-\mathrm{Th}(2)$ & $76.3(3)$ \\
\hline $\mathrm{C}(61)-\mathrm{C}(60)-\mathrm{Th}(2)$ & $75.6(3)$ \\
\hline $\mathrm{C}(65)-\mathrm{C}(60)-\mathrm{Th}(2)$ & $120.1(4)$ \\
\hline $\mathrm{C}(60)-\mathrm{C}(61)-\mathrm{C}(57)$ & $108.1(5)$ \\
\hline$C(60)-C(61)-C(66)$ & $125.6(6)$ \\
\hline $\mathrm{C}(57)-\mathrm{C}(61)-\mathrm{C}(66)$ & $126.0(6)$ \\
\hline $\mathrm{C}(60)-\mathrm{C}(61)-\mathrm{Th}(2)$ & $75.5(3)$ \\
\hline $\mathrm{C}(57)-\mathrm{C}(61)-\mathrm{Th}(2)$ & $76.6(3)$ \\
\hline $\mathrm{C}(66)-\mathrm{C}(61)-\mathrm{Th}(2)$ & $119.7(4)$ \\
\hline N(3)-C(67)-C(68) & $121.9(5)$ \\
\hline N(3)-C(67)-C(74) & $119.7(5)$ \\
\hline$C(68)-C(67)-C(74)$ & $118.3(5)$ \\
\hline $\mathrm{C}(73)-\mathrm{C}(68)-\mathrm{C}(69)$ & $117.2(6)$ \\
\hline$C(73)-C(68)-C(67)$ & $122.9(6)$ \\
\hline $\mathrm{C}(69)-\mathrm{C}(68)-\mathrm{C}(67)$ & $119.9(6)$ \\
\hline$C(70)-C(69)-C(68)$ & $121.8(7)$ \\
\hline $\mathrm{C}(71)-\mathrm{C}(70)-\mathrm{C}(69)$ & $120.1(7)$ \\
\hline $\mathrm{C}(70)-\mathrm{C}(71)-\mathrm{C}(72)$ & $119.9(7)$ \\
\hline$C(71)-C(72)-C(73)$ & $119.3(7)$ \\
\hline$C(68)-C(73)-C(72)$ & $121.7(7)$ \\
\hline
\end{tabular}




$\begin{array}{ll}\mathrm{C}(79)-\mathrm{C}(74)-\mathrm{C}(75) & 118.1(5) \\ \mathrm{C}(79)-\mathrm{C}(74)-\mathrm{C}(67) & 120.5(5) \\ \mathrm{C}(75)-\mathrm{C}(74)-\mathrm{C}(67) & 121.0(5) \\ \mathrm{C}(76)-\mathrm{C}(75)-\mathrm{C}(74) & 119.5(6) \\ \mathrm{C}(77)-\mathrm{C}(76)-\mathrm{C}(75) & 120.7(6) \\ \mathrm{C}(78)-\mathrm{C}(77)-\mathrm{C}(76) & 120.1(6) \\ \mathrm{C}(79)-\mathrm{C}(78)-\mathrm{C}(77) & 119.5(6) \\ \mathrm{C}(78)-\mathrm{C}(79)-\mathrm{C}(74) & 122.1(6) \\ \mathrm{N}(4)-\mathrm{C}(80)-\mathrm{C}(81) & 122.6(6) \\ \mathrm{N}(4)-\mathrm{C}(80)-\mathrm{C}(87) & 120.4(6) \\ \mathrm{C}(81)-\mathrm{C}(80)-\mathrm{C}(87) & 117.0(5) \\ \mathrm{C}(86)-\mathrm{C}(81)-\mathrm{C}(82) & 117.5(6) \\ \mathrm{C}(86)-\mathrm{C}(81)-\mathrm{C}(80) & 123.1(7) \\ \mathrm{C}(82)-\mathrm{C}(81)-\mathrm{C}(80) & 119.3(6) \\ \mathrm{C}(83)-\mathrm{C}(82)-\mathrm{C}(81) & 121.0(7) \\ \mathrm{C}(82)-\mathrm{C}(83)-\mathrm{C}(84) & 120.5(9) \\ \mathrm{C}(85)-\mathrm{C}(84)-\mathrm{C}(83) & 119.0(8) \\ \mathrm{C}(84)-\mathrm{C}(85)-\mathrm{C}(86) & 121.0(8) \\ \mathrm{C}(85)-\mathrm{C}(86)-\mathrm{C}(81) & 121.0(8) \\ \mathrm{C}(92)-\mathrm{C}(87)-\mathrm{C}(88) & 118.2(7) \\ \mathrm{C}(92)-\mathrm{C}(87)-\mathrm{C}(80) & 121.0(6) \\ \mathrm{C}(88)-\mathrm{C}(87)-\mathrm{C}(80) & 120.8(5) \\ \mathrm{C}(89)-\mathrm{C}(88)-\mathrm{C}(87) & 121.7(7) \\ \mathrm{C}(88)-\mathrm{C}(89)-\mathrm{C}(90) & 119.1(8) \\ \mathrm{C}(91)-\mathrm{C}(90)-\mathrm{C}(89) & 120.3(8) \\ \mathrm{C}(90)-\mathrm{C}(91)-\mathrm{C}(92) & 120.1(7) \\ \mathrm{C}(87)-\mathrm{C}(92)-\mathrm{C}(91) & 120.5(8) \\ & \end{array}$

Symmetry transformations used to generate equivalent atoms: 
Table S14. Anisotropic displacement parameters $\left(\AA^{2} \times 10^{3}\right)$ for $\left(\mathrm{C}_{5} \mathrm{Me}_{5}\right)_{2} \mathrm{Th}\left(-\mathrm{N}=\mathrm{CPh}_{2}\right)_{2}(\mathbf{1 0 a})$. The anisotropic displacement factor exponent takes the form: $-2 \pi^{2}\left[h^{2} a^{* 2} U^{11}+\ldots+2 h k a^{*} b^{*} U^{12}\right]$

\begin{tabular}{|c|c|c|c|c|c|c|}
\hline & $\mathrm{U}^{11}$ & $\mathrm{U}^{22}$ & $\mathrm{U}^{33}$ & $U^{23}$ & $\mathrm{U}^{13}$ & $\mathrm{U}^{12}$ \\
\hline $\operatorname{Th}(1)$ & $32(1)$ & $28(1)$ & $31(1)$ & $-14(1)$ & $0(1)$ & $-1(1)$ \\
\hline $\operatorname{Th}(2)$ & $29(1)$ & $26(1)$ & $29(1)$ & $-13(1)$ & $-2(1)$ & $-1(1)$ \\
\hline $\mathrm{N}(1)$ & $53(3)$ & $25(3)$ & $44(3)$ & $-18(2)$ & $1(2)$ & $-5(2)$ \\
\hline $\mathrm{N}(2)$ & $35(3)$ & $46(3)$ & $40(3)$ & $-16(3)$ & $-4(3)$ & $8(2)$ \\
\hline $\mathrm{N}(3)$ & $39(3)$ & $31(3)$ & $40(3)$ & $-17(2)$ & $-8(2)$ & $6(2)$ \\
\hline $\mathrm{N}(4)$ & $45(3)$ & $42(3)$ & $36(3)$ & $-12(2)$ & $-2(3)$ & $-15(3)$ \\
\hline$C(1)$ & $45(4)$ & $45(4)$ & $43(4)$ & $-28(3)$ & $7(3)$ & $-6(3)$ \\
\hline$C(2)$ & $61(5)$ & $56(4)$ & $35(4)$ & $-27(3)$ & $-4(3)$ & $9(4)$ \\
\hline$C(3)$ & $77(5)$ & $37(4)$ & $36(4)$ & $-20(3)$ & $23(4)$ & $-11(4)$ \\
\hline$C(4)$ & $40(4)$ & $50(4)$ & $47(4)$ & $-28(3)$ & $16(3)$ & $-10(3)$ \\
\hline$C(5)$ & $43(4)$ & $38(4)$ & $43(4)$ & $-22(3)$ & $4(3)$ & $4(3)$ \\
\hline$C(6)$ & $87(6)$ & $68(5)$ & $85(6)$ & $-58(5)$ & $18(5)$ & $-28(4)$ \\
\hline$C(7)$ & $84(6)$ & $113(7)$ & $56(5)$ & $-47(5)$ & $-17(4)$ & $12(5)$ \\
\hline$C(8)$ & $135(8)$ & $44(4)$ & $45(4)$ & $-11(4)$ & $32(5)$ & $-7(4)$ \\
\hline$C(9)$ & $66(5)$ & $87(6)$ & $95(6)$ & $-59(5)$ & $33(5)$ & $-35(5)$ \\
\hline$C(10)$ & $58(5)$ & $57(5)$ & $66(5)$ & $-30(4)$ & $5(4)$ & $13(4)$ \\
\hline$C(11)$ & $28(4)$ & $47(4)$ & $43(4)$ & $-9(3)$ & $4(3)$ & $4(3)$ \\
\hline $\mathrm{C}(12)$ & $28(4)$ & $56(4)$ & $44(4)$ & $-24(3)$ & $2(3)$ & $-7(3)$ \\
\hline$C(13)$ & $38(4)$ & $34(3)$ & $47(4)$ & $-12(3)$ & $14(3)$ & $-10(3)$ \\
\hline$C(14)$ & $30(4)$ & $52(4)$ & $39(4)$ & $-21(3)$ & $8(3)$ & $-11(3)$ \\
\hline$C(15)$ & $46(4)$ & $36(4)$ & $55(4)$ & $-26(3)$ & $21(3)$ & $-5(3)$ \\
\hline$C(16)$ & $56(5)$ & $69(5)$ & $71(5)$ & $4(4)$ & $-3(4)$ & $13(4)$ \\
\hline$C(17)$ & $51(5)$ & $121(7)$ & $79(6)$ & $-63(5)$ & $3(4)$ & $-31(5)$ \\
\hline $\mathrm{C}(18)$ & $58(5)$ & $40(4)$ & $95(6)$ & $-12(4)$ & $11(4)$ & $-10(4)$ \\
\hline$C(19)$ & $70(5)$ & $112(7)$ & $33(4)$ & $-28(4)$ & $8(4)$ & $-31(5)$ \\
\hline $\mathrm{C}(20)$ & $109(7)$ & $51(5)$ & $106(7)$ & $-53(5)$ & $60(6)$ & $-23(5)$ \\
\hline$C(21)$ & $41(4)$ & $42(4)$ & $31(3)$ & $-20(3)$ & $2(3)$ & $-2(3)$ \\
\hline $\mathrm{C}(22)$ & $38(4)$ & $33(3)$ & $30(3)$ & $-12(3)$ & $0(3)$ & $-7(3)$ \\
\hline$C(23)$ & $42(4)$ & 41(4) & $44(4)$ & $-22(3)$ & $-5(3)$ & $-2(3)$ \\
\hline $\mathrm{C}(24)$ & $49(4)$ & $39(4)$ & $48(4)$ & $-20(3)$ & $-7(3)$ & $5(3)$ \\
\hline$C(25)$ & $49(4)$ & $40(4)$ & $48(4)$ & $-7(3)$ & $-4(3)$ & $4(3)$ \\
\hline
\end{tabular}




\begin{tabular}{|c|c|c|c|c|c|c|}
\hline$C(26)$ & $60(5)$ & $52(4)$ & 49(4) & $-14(4)$ & $-17(4)$ & $-5(4)$ \\
\hline$C(27)$ & $62(5)$ & $35(4)$ & $42(4)$ & $-15(3)$ & $-8(3)$ & $-5(3)$ \\
\hline$C(28)$ & $37(4)$ & $37(3)$ & $40(4)$ & $-21(3)$ & $3(3)$ & $0(3)$ \\
\hline$C(29)$ & $47(4)$ & $42(4)$ & $60(4)$ & $-26(3)$ & $2(3)$ & $-5(3)$ \\
\hline$C(30)$ & $50(5)$ & $52(4)$ & $79(5)$ & $-33(4)$ & $12(4)$ & $-20(4)$ \\
\hline$C(31)$ & $43(5)$ & $83(6)$ & $90(6)$ & $-52(5)$ & $3(4)$ & $-19(4)$ \\
\hline$C(32)$ & $49(5)$ & $80(6)$ & $69(5)$ & $-39(5)$ & $-18(4)$ & $5(4)$ \\
\hline$C(33)$ & $55(5)$ & 41(4) & $53(4)$ & $-28(3)$ & $-5(3)$ & $-3(3)$ \\
\hline$C(34)$ & $34(4)$ & $43(4)$ & $35(4)$ & $-15(3)$ & $5(3)$ & $4(3)$ \\
\hline$C(35)$ & $33(4)$ & $46(4)$ & $44(4)$ & $-18(3)$ & $5(3)$ & $5(3)$ \\
\hline$C(36)$ & $53(5)$ & $52(4)$ & $50(4)$ & $-20(4)$ & $6(3)$ & $-3(4)$ \\
\hline$C(37)$ & $76(6)$ & $50(5)$ & $64(5)$ & $-26(4)$ & $23(4)$ & $-9(4)$ \\
\hline$C(38)$ & $79(6)$ & $48(5)$ & $65(6)$ & $-13(4)$ & $28(5)$ & $10(4)$ \\
\hline$C(39)$ & $65(6)$ & $69(6)$ & $47(5)$ & $-4(4)$ & $3(4)$ & $24(4)$ \\
\hline $\mathrm{C}(40)$ & $46(5)$ & $55(5)$ & $41(4)$ & $-3(3)$ & $-5(3)$ & $13(3)$ \\
\hline $\mathrm{C}(41)$ & $35(4)$ & $50(4)$ & $39(4)$ & $-11(3)$ & $-3(3)$ & $-4(3)$ \\
\hline$C(42)$ & $35(5)$ & $96(6)$ & $70(5)$ & $-42(5)$ & $4(4)$ & $-7(4)$ \\
\hline$C(43)$ & $38(5)$ & $123(8)$ & $76(6)$ & $-43(6)$ & $12(4)$ & $-21(5)$ \\
\hline$C(44)$ & $70(6)$ & $93(6)$ & $69(5)$ & $-31(5)$ & $2(5)$ & $-41(5)$ \\
\hline$C(45)$ & $79(7)$ & $77(6)$ & $92(6)$ & $-51(5)$ & $2(5)$ & $-23(5)$ \\
\hline$C(46)$ & $51(5)$ & $64(5)$ & $73(5)$ & $-30(4)$ & $-5(4)$ & $-9(4)$ \\
\hline$C(47)$ & $43(4)$ & $38(4)$ & $38(3)$ & $-21(3)$ & $-9(3)$ & $-2(3)$ \\
\hline$C(48)$ & $55(5)$ & $39(4)$ & $45(4)$ & $-29(3)$ & $-11(3)$ & $6(3)$ \\
\hline C(49) & $53(4)$ & $51(4)$ & $33(4)$ & $-26(3)$ & $4(3)$ & $-11(3)$ \\
\hline$C(50)$ & $62(5)$ & $39(4)$ & $34(4)$ & $-18(3)$ & $-14(3)$ & $-1(3)$ \\
\hline$C(51)$ & $40(4)$ & $46(4)$ & $39(4)$ & $-25(3)$ & $-9(3)$ & $0(3)$ \\
\hline$C(52)$ & $72(5)$ & $50(4)$ & $66(5)$ & $-27(4)$ & $-12(4)$ & $-16(4)$ \\
\hline$C(53)$ & $69(5)$ & $56(4)$ & $89(6)$ & $-50(4)$ & $-25(4)$ & $17(4)$ \\
\hline$C(54)$ & $75(6)$ & $102(6)$ & $51(5)$ & $-45(4)$ & $15(4)$ & $-24(5)$ \\
\hline$C(55)$ & $100(6)$ & $47(4)$ & $38(4)$ & $-11(3)$ & $-29(4)$ & $-5(4)$ \\
\hline$C(56)$ & $55(5)$ & $65(5)$ & $68(5)$ & $-39(4)$ & $-21(4)$ & $4(4)$ \\
\hline$C(57)$ & $30(4)$ & $36(3)$ & $47(4)$ & $-11(3)$ & $-13(3)$ & $-1(3)$ \\
\hline$C(58)$ & $37(4)$ & $37(4)$ & $43(4)$ & $-18(3)$ & $-6(3)$ & $2(3)$ \\
\hline $\mathrm{C}(59)$ & $28(4)$ & $41(4)$ & $41(4)$ & $-20(3)$ & $-4(3)$ & $-3(3)$ \\
\hline$C(60)$ & $34(4)$ & $38(3)$ & $43(4)$ & $-22(3)$ & $-11(3)$ & 1(3) \\
\hline $\mathrm{C}(61)$ & $36(4)$ & $54(4)$ & $38(4)$ & $-23(3)$ & $-4(3)$ & $-6(3)$ \\
\hline
\end{tabular}




\begin{tabular}{|c|c|c|c|c|c|c|}
\hline$C(62)$ & $62(5)$ & $45(4)$ & $64(5)$ & 1(4) & $-23(4)$ & $-7(4)$ \\
\hline$C(63)$ & $39(4)$ & $60(4)$ & $80(5)$ & $-40(4)$ & $-10(4)$ & $10(3)$ \\
\hline$C(64)$ & $47(4)$ & $53(4)$ & $55(4)$ & $-20(3)$ & $5(3)$ & $-12(3)$ \\
\hline$C(65)$ & $50(5)$ & $56(4)$ & $79(5)$ & $-43(4)$ & $-20(4)$ & $3(3)$ \\
\hline C(66) & $46(4)$ & $102(6)$ & $45(4)$ & $-41(4)$ & $-1(3)$ & $-8(4)$ \\
\hline$C(67)$ & $40(4)$ & $32(3)$ & $36(3)$ & $-19(3)$ & $-7(3)$ & $0(3)$ \\
\hline $\mathrm{C}(68)$ & $39(4)$ & $42(4)$ & $40(4)$ & $-24(3)$ & $-9(3)$ & $0(3)$ \\
\hline $\mathrm{C}(69)$ & $49(5)$ & $60(4)$ & $53(4)$ & $-37(4)$ & $-7(3)$ & $-3(4)$ \\
\hline$C(70)$ & $40(5)$ & $77(5)$ & $64(5)$ & $-43(4)$ & $0(4)$ & $-7(4)$ \\
\hline$C(71)$ & $40(5)$ & $99(7)$ & $88(6)$ & $-67(6)$ & $-23(4)$ & $19(5)$ \\
\hline$C(72)$ & $62(6)$ & $61(5)$ & $81(6)$ & $-36(4)$ & $-27(5)$ & $23(4)$ \\
\hline$C(73)$ & $44(5)$ & $54(4)$ & $50(4)$ & $-22(4)$ & $-15(3)$ & $7(3)$ \\
\hline$C(74)$ & $37(4)$ & 27(3) & $40(4)$ & $-13(3)$ & $-8(3)$ & $5(3)$ \\
\hline$C(75)$ & $51(4)$ & $37(4)$ & $49(4)$ & $-20(3)$ & $-11(3)$ & $3(3)$ \\
\hline$C(76)$ & $72(5)$ & $41(4)$ & $58(5)$ & $-20(4)$ & $-16(4)$ & $-12(4)$ \\
\hline$C(77)$ & $48(5)$ & $46(4)$ & $69(5)$ & $2(4)$ & $-19(4)$ & $-14(4)$ \\
\hline$C(78)$ & $50(5)$ & $42(4)$ & $57(4)$ & $-12(3)$ & $1(3)$ & $-3(3)$ \\
\hline$C(79)$ & $51(4)$ & $36(4)$ & $47(4)$ & $-17(3)$ & $-5(3)$ & $3(3)$ \\
\hline $\mathrm{C}(80)$ & $45(4)$ & $52(4)$ & $30(3)$ & $-13(3)$ & $-8(3)$ & $-19(3)$ \\
\hline$C(81)$ & $44(4)$ & $51(4)$ & $40(4)$ & $-13(3)$ & $-9(3)$ & $-20(3)$ \\
\hline$C(82)$ & $63(5)$ & $53(5)$ & $51(4)$ & $-9(4)$ & $-17(4)$ & $-17(4)$ \\
\hline$C(83)$ & $92(7)$ & $57(5)$ & $67(5)$ & $-19(4)$ & $-34(5)$ & $-14(4)$ \\
\hline$C(84)$ & $128(9)$ & $54(5)$ & $57(6)$ & $1(4)$ & $-38(5)$ & $-43(6)$ \\
\hline$C(85)$ & $120(9)$ & $83(7)$ & $45(5)$ & $-4(5)$ & $-4(5)$ & $-63(6)$ \\
\hline$C(86)$ & $69(5)$ & $75(5)$ & $39(4)$ & $-17(4)$ & $0(4)$ & $-35(4)$ \\
\hline$C(87)$ & $36(4)$ & $68(5)$ & $30(4)$ & $-14(3)$ & $-2(3)$ & $-9(3)$ \\
\hline$C(88)$ & $44(5)$ & $80(5)$ & $69(5)$ & $-34(4)$ & $5(4)$ & $-12(4)$ \\
\hline $\mathrm{C}(89)$ & $60(6)$ & $90(6)$ & $87(6)$ & $-44(5)$ & $9(5)$ & $0(5)$ \\
\hline$C(90)$ & $47(6)$ & $129(8)$ & $65(6)$ & $-38(6)$ & $-1(4)$ & $19(5)$ \\
\hline $\mathrm{C}(91)$ & $36(5)$ & $148(9)$ & $70(6)$ & $-47(6)$ & $-9(4)$ & $-9(5)$ \\
\hline$C(92)$ & $41(5)$ & $115(7)$ & $64(5)$ & $-39(5)$ & $-12(4)$ & $-10(5)$ \\
\hline
\end{tabular}


Table S15. Hydrogen coordinates ( x 104) and isotropic displacement parameters $\left(\AA^{2} \times 10^{3}\right)$ for $\left(\mathrm{C}_{5} \mathrm{Me}_{5}\right)_{2} \mathrm{Th}\left(-\mathrm{N}=\mathrm{CPh}_{2}\right)_{2}$ (10a).

\begin{tabular}{|c|c|c|c|c|}
\hline & $\mathrm{x}$ & $\mathrm{y}$ & z & $\mathrm{U}(\mathrm{eq})$ \\
\hline $\mathrm{H}(6 \mathrm{~A})$ & 2475 & 6596 & 3642 & 107 \\
\hline $\mathrm{H}(6 \mathrm{~B})$ & 1110 & 7003 & 3617 & 107 \\
\hline $\mathrm{H}(6 \mathrm{C})$ & 1811 & 6975 & 2911 & 107 \\
\hline $\mathrm{H}(7 \mathrm{~A})$ & 2801 & 4560 & 4787 & 122 \\
\hline $\mathrm{H}(7 \mathrm{~B})$ & 2623 & 5320 & 4822 & 122 \\
\hline $\mathrm{H}(7 \mathrm{C})$ & 3448 & 5228 & 4176 & 122 \\
\hline $\mathrm{H}(8 \mathrm{~A})$ & 1444 & 3868 & 4934 & 122 \\
\hline $\mathrm{H}(8 \mathrm{~B})$ & 606 & 3714 & 4447 & 122 \\
\hline $\mathrm{H}(8 \mathrm{C})$ & -43 & 3951 & 5049 & 122 \\
\hline $\mathrm{H}(9 \mathrm{~A})$ & -1479 & 4416 & 3886 & 114 \\
\hline $\mathrm{H}(9 \mathrm{~B})$ & -1566 & 5058 & 3109 & 114 \\
\hline $\mathrm{H}(9 \mathrm{C})$ & -2260 & 5160 & 3782 & 114 \\
\hline $\mathrm{H}(10 \mathrm{~A})$ & -598 & 7032 & 2787 & 93 \\
\hline $\mathrm{H}(10 \mathrm{~B})$ & -1740 & 6597 & 3261 & 93 \\
\hline $\mathrm{H}(10 \mathrm{C})$ & -1213 & 6522 & 2547 & 93 \\
\hline $\mathrm{H}(16 \mathrm{~A})$ & 5430 & 4566 & 3434 & 119 \\
\hline $\mathrm{H}(16 \mathrm{~B})$ & 5873 & 4136 & 2961 & 119 \\
\hline $\mathrm{H}(16 \mathrm{C})$ & 4583 & 3991 & 3438 & 119 \\
\hline $\mathrm{H}(17 \mathrm{~A})$ & 5279 & 5659 & 3200 & 112 \\
\hline $\mathrm{H}(17 \mathrm{~B})$ & 4290 & 6346 & 2910 & 112 \\
\hline $\mathrm{H}(17 \mathrm{C})$ & 5593 & 6328 & 2466 & 112 \\
\hline $\mathrm{H}(18 \mathrm{~A})$ & 3132 & 7053 & 894 & 107 \\
\hline $\mathrm{H}(18 \mathrm{~B})$ & 4390 & 7107 & 1186 & 107 \\
\hline $\mathrm{H}(18 \mathrm{C})$ & 3084 & 7120 & 1628 & 107 \\
\hline $\mathrm{H}(19 \mathrm{~A})$ & 2726 & 6317 & 515 & 107 \\
\hline $\mathrm{H}(19 \mathrm{~B})$ & 2208 & 5546 & 814 & 107 \\
\hline $\mathrm{H}(19 \mathrm{C})$ & 3584 & 5664 & 435 & 107 \\
\hline $\mathrm{H}(20 \mathrm{~A})$ & 4259 & 3786 & 2275 & 129 \\
\hline $\mathrm{H}(20 \mathrm{~B})$ & 4409 & 4233 & 1432 & 129 \\
\hline $\mathrm{H}(20 \mathrm{C})$ & 3050 & 4120 & 1834 & 129 \\
\hline
\end{tabular}




\begin{tabular}{|c|c|c|c|c|}
\hline $\mathrm{H}(23)$ & 2327 & 2217 & 3035 & 49 \\
\hline $\mathrm{H}(24)$ & 4088 & 1405 & 3361 & 55 \\
\hline $\mathrm{H}(25)$ & 5433 & 1445 & 4144 & 60 \\
\hline $\mathrm{H}(26)$ & 4987 & 2331 & 4578 & 66 \\
\hline $\mathrm{H}(27)$ & 3250 & 3155 & 4241 & 56 \\
\hline $\mathrm{H}(29)$ & 416 & 2109 & 4046 & 58 \\
\hline $\mathrm{H}(30)$ & -1564 & 1800 & 3934 & 70 \\
\hline $\mathrm{H}(31)$ & -2870 & 2627 & 3091 & 79 \\
\hline $\mathrm{H}(32)$ & -2258 & 3783 & 2336 & 75 \\
\hline $\mathrm{H}(33)$ & -318 & 4106 & 2439 & 56 \\
\hline $\mathrm{H}(36)$ & 715 & 7222 & 1571 & 64 \\
\hline $\mathrm{H}(37)$ & 765 & 8465 & 896 & 78 \\
\hline $\mathrm{H}(38)$ & -223 & 9047 & -169 & 87 \\
\hline H(39) & -1293 & 8378 & -555 & 85 \\
\hline $\mathrm{H}(40)$ & -1352 & 7135 & 114 & 66 \\
\hline $\mathrm{H}(42)$ & -2794 & 6351 & 1286 & 78 \\
\hline $\mathrm{H}(43)$ & -4085 & 5745 & 957 & 95 \\
\hline $\mathrm{H}(44)$ & -3203 & 4861 & 565 & 91 \\
\hline $\mathrm{H}(45)$ & -1038 & 4575 & 555 & 92 \\
\hline $\mathrm{H}(46)$ & 245 & 5167 & 916 & 75 \\
\hline $\mathrm{H}(52 \mathrm{~A})$ & 14354 & 7910 & 2300 & 90 \\
\hline $\mathrm{H}(52 \mathrm{~B})$ & 13204 & 8334 & 1832 & 90 \\
\hline $\mathrm{H}(52 \mathrm{C})$ & 13376 & 8432 & 2530 & 90 \\
\hline $\mathrm{H}(53 \mathrm{~A})$ & 16327 & 7836 & 1986 & 98 \\
\hline $\mathrm{H}(53 \mathrm{~B})$ & 17529 & 8271 & 1796 & 98 \\
\hline $\mathrm{H}(53 \mathrm{C})$ & 16951 & 8156 & 1192 & 98 \\
\hline $\mathrm{H}(54 \mathrm{~A})$ & 17393 & 10372 & 211 & 107 \\
\hline $\mathrm{H}(54 \mathrm{~B})$ & 17639 & 9592 & 221 & 107 \\
\hline $\mathrm{H}(54 \mathrm{C})$ & 18103 & 9732 & 841 & 107 \\
\hline $\mathrm{H}(55 \mathrm{~A})$ & 14058 & 11133 & 403 & 93 \\
\hline $\mathrm{H}(55 \mathrm{~B})$ & 15027 & 10950 & -147 & 93 \\
\hline $\mathrm{H}(55 \mathrm{C})$ & 15512 & 11198 & 394 & 93 \\
\hline $\mathrm{H}(56 \mathrm{~A})$ & 12623 & 10511 & 1168 & 87 \\
\hline $\mathrm{H}(56 \mathrm{~B})$ & 12524 & 9882 & 1954 & 87 \\
\hline $\mathrm{H}(56 \mathrm{C})$ & 12184 & 9757 & 1298 & 87 \\
\hline $\mathrm{H}(62 \mathrm{~A})$ & 17070 & 8090 & 4262 & 96 \\
\hline
\end{tabular}




\begin{tabular}{|c|c|c|c|c|}
\hline $\mathrm{H}(62 \mathrm{~B})$ & 18477 & 7925 & 4004 & 96 \\
\hline $\mathrm{H}(62 \mathrm{C})$ & 17348 & 7882 & 3605 & 96 \\
\hline $\mathrm{H}(63 \mathrm{~A})$ & 19685 & 9109 & 1881 & 86 \\
\hline $\mathrm{H}(63 \mathrm{~B})$ & 18831 & 8455 & 2265 & 86 \\
\hline $\mathrm{H}(63 \mathrm{C})$ & 19961 & 8507 & 2658 & 86 \\
\hline $\mathrm{H}(64 \mathrm{~A})$ & 19538 & 10225 & 1502 & 79 \\
\hline $\mathrm{H}(64 \mathrm{~B})$ & 19688 & 10712 & 1899 & 79 \\
\hline $\mathrm{H}(64 \mathrm{C})$ & 18521 & 10878 & 1437 & 79 \\
\hline $\mathrm{H}(65 \mathrm{~A})$ & 16768 & 10929 & 3308 & 84 \\
\hline $\mathrm{H}(65 \mathrm{~B})$ & 16956 & 11276 & 2460 & 84 \\
\hline $\mathrm{H}(65 \mathrm{C})$ & 18126 & 11105 & 2922 & 84 \\
\hline $\mathrm{H}(66 \mathrm{~A})$ & 16149 & 9961 & 4147 & 92 \\
\hline $\mathrm{H}(66 \mathrm{~B})$ & 16940 & 9220 & 4580 & 92 \\
\hline $\mathrm{H}(66 \mathrm{C})$ & 15679 & 9221 & 4258 & 92 \\
\hline $\mathrm{H}(69)$ & 12684 & 10880 & 2506 & 59 \\
\hline $\mathrm{H}(70)$ & 10579 & 11275 & 2610 & 67 \\
\hline $\mathrm{H}(71)$ & 9853 & 12465 & 1911 & 80 \\
\hline $\mathrm{H}(72)$ & 11260 & 13273 & 1101 & 80 \\
\hline $\mathrm{H}(73)$ & 13385 & 12868 & 1001 & 59 \\
\hline $\mathrm{H}(75)$ & 14933 & 12744 & 1918 & 54 \\
\hline $\mathrm{H}(76)$ & 16627 & 13456 & 1575 & 67 \\
\hline $\mathrm{H}(77)$ & 18311 & 13281 & 833 & 74 \\
\hline $\mathrm{H}(78)$ & 18343 & 12371 & 446 & 64 \\
\hline $\mathrm{H}(79)$ & 16704 & 11645 & 804 & 55 \\
\hline $\mathrm{H}(82)$ & 15221 & 7655 & 3542 & 70 \\
\hline $\mathrm{H}(83)$ & 15466 & 6419 & 4240 & 86 \\
\hline $\mathrm{H}(84)$ & 14144 & 5884 & 5274 & 98 \\
\hline $\mathrm{H}(85)$ & 12598 & 6607 & 5604 & 103 \\
\hline H(86) & 12302 & 7836 & 4898 & 74 \\
\hline $\mathrm{H}(88)$ & 13507 & 9816 & 4080 & 77 \\
\hline H(89) & 11804 & 10515 & 4315 & 95 \\
\hline $\mathrm{H}(90)$ & 9759 & 10311 & 4205 & 101 \\
\hline $\mathrm{H}(91)$ & 9451 & 9400 & 3891 & 101 \\
\hline $\mathrm{H}(92)$ & 11171 & 8659 & 3707 & 86 \\
\hline
\end{tabular}


Table S16. Crystal data and structure refinement for $\left(\mathrm{C}_{5} \mathrm{Me}_{5}\right)_{2} \mathrm{Th}[-\mathrm{N}=\mathrm{C}(\mathrm{Ph})(\mathrm{Bz})]_{2}(\mathbf{1 0 b})$.

Identification code

Empirical formula

Formula weight

Temperature

Wavelength

Crystal system

Space group

Unit cell dimensions

Volume

$\mathrm{Z}$

Density (calculated)

Absorption coefficient

$\mathrm{F}(000)$

Crystal size

Theta range for data collection

Index ranges

Reflections collected

Independent reflections

Max. and min. transmission

Refinement method

Data / restraints / parameters

Goodness-of-fit on $\mathrm{F}^{2}$

Final R indices [I $>2 \operatorname{sigma}(\mathrm{I})]$

$\mathrm{R}$ indices (all data)

Largest diff. peak and hole $\operatorname{ccd} 777 \mathrm{~m}$

$\mathrm{C}_{48} \mathrm{H}_{54} \mathrm{~N}_{2} \mathrm{Th}$

890.97

203(2) K

$0.71073 \AA$

monoclinic

$\mathrm{C} 2 / \mathrm{c}$

$\mathrm{a}=15.416(5) \AA$

$\alpha=90^{\circ}$.

$\mathrm{b}=13.461(5) \AA$

$\beta=94.673(6)^{\circ}$.

$\mathrm{c}=19.347(6) \AA$

$\gamma=90^{\circ}$.

4001(2) $\AA^{3}$

4

$1.479 \mathrm{Mg} / \mathrm{m}^{3}$

$3.761 \mathrm{~mm}^{-1}$

1784

$0.10 \times 0.08 \times 0.08 \mathrm{~mm}^{3}$

2.01 to $22.46^{\circ}$.

$-16<=\mathrm{h}<=16,-14<=\mathrm{k}<=14,-17<=1<=20$

9973

$2597[\mathrm{R}(\mathrm{int})=0.0948]$

0.7529 and 0.7048

Full-matrix least-squares on $\mathrm{F}^{2}$

$2597 / 0 / 231$

1.273

$\mathrm{R} 1=0.0615, \mathrm{wR} 2=0.1135$

$\mathrm{R} 1=0.0766, \mathrm{wR} 2=0.1172$

1.831 and -1.973 e. $\AA^{-3}$ 
Table S17. Atomic coordinates ( x 104) and equivalent isotropic displacement parameters $\left(\AA^{2} \times 10^{3}\right)$ for $\left(\mathrm{C}_{5} \mathrm{Me}_{5}\right)_{2} \mathrm{Th}[-\mathrm{N}=\mathrm{C}(\mathrm{Ph})(\mathrm{Bz})]_{2}(\mathbf{1 0 b}) . \mathrm{U}(\mathrm{eq})$ is defined as one third of the trace of the orthogonalized $\mathrm{U}^{\mathrm{ij}}$ tensor.

\begin{tabular}{|c|c|c|c|c|}
\hline & $\mathrm{x}$ & $\mathrm{y}$ & z & $\mathrm{U}(\mathrm{eq})$ \\
\hline $\mathrm{N}(1)$ & $-4226(5)$ & $599(6)$ & $3223(4)$ & $43(2)$ \\
\hline $\operatorname{Th}(1)$ & -5000 & $1644(1)$ & 2500 & $31(1)$ \\
\hline $\mathrm{C}(1)$ & $-4061(6)$ & $1783(8)$ & $1299(5)$ & $41(3)$ \\
\hline$C(2)$ & $-3441(6)$ & $1508(8)$ & $1846(6)$ & $44(3)$ \\
\hline$C(3)$ & $-3320(7)$ & $2323(8)$ & $2307(6)$ & $46(3)$ \\
\hline$C(4)$ & $-3874(6)$ & $3103(7)$ & $2045(6)$ & $43(3)$ \\
\hline$C(5)$ & $-4345(6)$ & $2758(7)$ & $1424(5)$ & $37(2)$ \\
\hline$C(6)$ & $-4317(7)$ & 1191(9) & $657(6)$ & $53(3)$ \\
\hline$C(7)$ & $-2917(7)$ & $554(9)$ & 1889(7) & $63(4)$ \\
\hline $\mathrm{C}(8)$ & $-2698(7)$ & $2367(11)$ & $2945(6)$ & $67(4)$ \\
\hline$C(9)$ & $-3863(8)$ & $4142(9)$ & $2320(7)$ & $73(4)$ \\
\hline$C(10)$ & $-4983(7)$ & $3347(9)$ & $978(6)$ & $58(3)$ \\
\hline$C(11)$ & $-3790(6)$ & $-147(8)$ & $3447(5)$ & $41(3)$ \\
\hline$C(12)$ & $-3152(6)$ & $-119(8)$ & $4084(5)$ & $42(3)$ \\
\hline$C(13)$ & $-2612(7)$ & $-904(9)$ & $4299(7)$ & $59(3)$ \\
\hline$C(14)$ & $-2020(8)$ & $-828(12)$ & $4866(7)$ & $69(4)$ \\
\hline$C(15)$ & $-1943(8)$ & $60(13)$ & $5228(7)$ & $70(4)$ \\
\hline$C(16)$ & $-2456(8)$ & $829(11)$ & $5034(6)$ & $66(4)$ \\
\hline$C(17)$ & $-3065(7)$ & $774(9)$ & $4473(6)$ & $52(3)$ \\
\hline$C(18)$ & $-3895(7)$ & $-1144(8)$ & $3079(6)$ & $50(3)$ \\
\hline$C(19)$ & $-4425(7)$ & $-1912(7)$ & $3429(6)$ & $43(3)$ \\
\hline$C(20)$ & $-4257(8)$ & $-2911(9)$ & $3328(8)$ & $67(4)$ \\
\hline $\mathrm{C}(21)$ & $-4774(11)$ & $-3627(9)$ & $3630(9)$ & $84(5)$ \\
\hline $\mathrm{C}(22)$ & $-5476(9)$ & $-3365(11)$ & $3957(7)$ & $66(4)$ \\
\hline$C(23)$ & $-5648(8)$ & $-2391(10)$ & $4050(7)$ & $61(3)$ \\
\hline$C(24)$ & $-5135(8)$ & $-1680(9)$ & $3784(6)$ & $57(3)$ \\
\hline
\end{tabular}


Table S18. Bond lengths $[\AA]$ and angles $\left[^{\circ}\right]$ for $\left(\mathrm{C}_{5} \mathrm{Me}_{5}\right)_{2} \mathrm{Th}[-\mathrm{N}=\mathrm{C}(\mathrm{Ph})(\mathrm{Bz})]_{2}(\mathbf{1 0 b})$..

\begin{tabular}{|c|c|}
\hline $\mathrm{N}(1)-\mathrm{C}(11)$ & $1.264(13)$ \\
\hline $\mathrm{N}(1)-\operatorname{Th}(1)$ & $2.256(8)$ \\
\hline $\operatorname{Th}(1)-\mathrm{N}(1) \# 1$ & $2.256(8)$ \\
\hline $\operatorname{Th}(1)-\mathrm{C}(3)$ & $2.800(10)$ \\
\hline $\operatorname{Th}(1)-\mathrm{C}(3) \# 1$ & $2.800(10)$ \\
\hline $\operatorname{Th}(1)-\mathrm{C}(2) \# 1$ & $2.811(9)$ \\
\hline $\operatorname{Th}(1)-C(2)$ & $2.811(9)$ \\
\hline $\operatorname{Th}(1)-C(4) \# 1$ & $2.810(10)$ \\
\hline $\operatorname{Th}(1)-C(4)$ & $2.810(10)$ \\
\hline $\operatorname{Th}(1)-C(5) \# 1$ & $2.819(10)$ \\
\hline $\operatorname{Th}(1)-C(5)$ & $2.819(10)$ \\
\hline $\operatorname{Th}(1)-C(1) \# 1$ & $2.842(10)$ \\
\hline $\operatorname{Th}(1)-C(1)$ & $2.842(10)$ \\
\hline$C(1)-C(5)$ & $1.410(14)$ \\
\hline$C(1)-C(2)$ & $1.415(15)$ \\
\hline$C(1)-C(6)$ & $1.502(15)$ \\
\hline $\mathrm{C}(2)-\mathrm{C}(3)$ & $1.416(15)$ \\
\hline$C(2)-C(7)$ & $1.516(14)$ \\
\hline$C(3)-C(4)$ & $1.420(14)$ \\
\hline$C(3)-C(8)$ & $1.501(15)$ \\
\hline$C(4)-C(5)$ & $1.430(15)$ \\
\hline$C(4)-C(9)$ & $1.496(16)$ \\
\hline$C(5)-C(10)$ & $1.484(14)$ \\
\hline$C(11)-C(12)$ & $1.513(14)$ \\
\hline$C(11)-C(18)$ & $1.522(15)$ \\
\hline$C(12)-C(13)$ & $1.389(15)$ \\
\hline$C(12)-C(17)$ & $1.419(15)$ \\
\hline$C(13)-C(14)$ & $1.373(17)$ \\
\hline$C(14)-C(15)$ & $1.386(19)$ \\
\hline$C(15)-C(16)$ & $1.337(18)$ \\
\hline$C(16)-C(17)$ & $1.378(16)$ \\
\hline$C(18)-C(19)$ & $1.512(15)$ \\
\hline C(19)-C(24) & $1.375(16)$ \\
\hline C(19)-C(20) & $1.387(15)$ \\
\hline
\end{tabular}




\begin{tabular}{|c|c|}
\hline$C(20)-C(21)$ & $1.407(19)$ \\
\hline$C(21)-C(22)$ & $1.35(2)$ \\
\hline$C(22)-C(23)$ & $1.352(17)$ \\
\hline$C(23)-C(24)$ & $1.368(17)$ \\
\hline $\mathrm{C}(11)-\mathrm{N}(1)-\mathrm{Th}(1)$ & $161.2(8)$ \\
\hline $\mathrm{N}(1) \# 1-\mathrm{Th}(1)-\mathrm{N}(1)$ & $102.9(4)$ \\
\hline $\mathrm{N}(1) \# 1-\mathrm{Th}(1)-\mathrm{C}(3)$ & $124.7(3)$ \\
\hline $\mathrm{N}(1)-\mathrm{Th}(1)-\mathrm{C}(3)$ & $80.7(3)$ \\
\hline $\mathrm{N}(1) \# 1-\mathrm{Th}(1)-\mathrm{C}(3) \# 1$ & $80.7(3)$ \\
\hline $\mathrm{N}(1)-\mathrm{Th}(1)-\mathrm{C}(3) \# 1$ & $124.7(3)$ \\
\hline $\mathrm{C}(3)-\mathrm{Th}(1)-\mathrm{C}(3) \# 1$ & $141.9(5)$ \\
\hline $\mathrm{N}(1) \# 1-\mathrm{Th}(1)-\mathrm{C}(2) \# 1$ & $79.1(3)$ \\
\hline $\mathrm{N}(1)-\mathrm{Th}(1)-\mathrm{C}(2) \# 1$ & $96.2(3)$ \\
\hline $\mathrm{C}(3)-\mathrm{Th}(1)-\mathrm{C}(2) \# 1$ & $156.2(3)$ \\
\hline $\mathrm{C}(3) \# 1-\mathrm{Th}(1)-\mathrm{C}(2) \# 1$ & $29.2(3)$ \\
\hline $\mathrm{N}(1) \# 1-\mathrm{Th}(1)-\mathrm{C}(2)$ & $96.2(3)$ \\
\hline $\mathrm{N}(1)-\mathrm{Th}(1)-\mathrm{C}(2)$ & $79.1(3)$ \\
\hline $\mathrm{C}(3)-\mathrm{Th}(1)-\mathrm{C}(2)$ & $29.2(3)$ \\
\hline $\mathrm{C}(3) \# 1-\mathrm{Th}(1)-\mathrm{C}(2)$ & $156.2(3)$ \\
\hline $\mathrm{C}(2) \# 1-\mathrm{Th}(1)-\mathrm{C}(2)$ & $172.6(4)$ \\
\hline $\mathrm{N}(1) \# 1-\mathrm{Th}(1)-\mathrm{C}(4) \# 1$ & $108.7(3)$ \\
\hline $\mathrm{N}(1)-\mathrm{Th}(1)-\mathrm{C}(4) \# 1$ & $123.4(3)$ \\
\hline $\mathrm{C}(3)-\mathrm{Th}(1)-\mathrm{C}(4) \# 1$ & $114.5(3)$ \\
\hline $\mathrm{C}(3) \# 1-\mathrm{Th}(1)-\mathrm{C}(4) \# 1$ & 29.3(3) \\
\hline $\mathrm{C}(2) \# 1-\mathrm{Th}(1)-\mathrm{C}(4) \# 1$ & $48.1(3)$ \\
\hline $\mathrm{C}(2)-\mathrm{Th}(1)-\mathrm{C}(4) \# 1$ & $139.4(3)$ \\
\hline $\mathrm{N}(1) \# 1-\mathrm{Th}(1)-\mathrm{C}(4)$ & $123.4(3)$ \\
\hline $\mathrm{N}(1)-\mathrm{Th}(1)-\mathrm{C}(4)$ & $108.7(3)$ \\
\hline$C(3)-\operatorname{Th}(1)-C(4)$ & 29.3(3) \\
\hline $\mathrm{C}(3) \# 1-\mathrm{Th}(1)-\mathrm{C}(4)$ & $114.5(3)$ \\
\hline $\mathrm{C}(2) \# 1-\mathrm{Th}(1)-\mathrm{C}(4)$ & $139.4(3)$ \\
\hline $\mathrm{C}(2)-\mathrm{Th}(1)-\mathrm{C}(4)$ & $48.1(3)$ \\
\hline C(4)\#1-Th(1)-C(4) & $91.3(4)$ \\
\hline $\mathrm{N}(1) \# 1-\mathrm{Th}(1)-\mathrm{C}(5) \# 1$ & $125.9(3)$ \\
\hline $\mathrm{N}(1)-\mathrm{Th}(1)-\mathrm{C}(5) \# 1$ & $94.4(3)$ \\
\hline
\end{tabular}




\begin{tabular}{|c|c|}
\hline$C(3)-T h(1)-C(5) \# 1$ & $108.4(3)$ \\
\hline $\mathrm{C}(3) \# 1-\mathrm{Th}(1)-\mathrm{C}(5) \# 1$ & $48.5(3)$ \\
\hline $\mathrm{C}(2) \# 1-\mathrm{Th}(1)-\mathrm{C}(5) \# 1$ & $48.0(3)$ \\
\hline $\mathrm{C}(2)-\mathrm{Th}(1)-\mathrm{C}(5) \# 1$ & $137.5(3)$ \\
\hline $\mathrm{C}(4) \# 1-\mathrm{Th}(1)-\mathrm{C}(5) \# 1$ & $29.4(3)$ \\
\hline $\mathrm{C}(4)-\mathrm{Th}(1)-\mathrm{C}(5) \# 1$ & $97.3(3)$ \\
\hline $\mathrm{N}(1) \# 1-\mathrm{Th}(1)-\mathrm{C}(5)$ & $94.4(3)$ \\
\hline $\mathrm{N}(1)-\mathrm{Th}(1)-\mathrm{C}(5)$ & $125.9(3)$ \\
\hline $\mathrm{C}(3)-\mathrm{Th}(1)-\mathrm{C}(5)$ & $48.5(3)$ \\
\hline $\mathrm{C}(3) \# 1-\mathrm{Th}(1)-\mathrm{C}(5)$ & $108.4(3)$ \\
\hline $\mathrm{C}(2) \# 1-\mathrm{Th}(1)-\mathrm{C}(5)$ & $137.5(3)$ \\
\hline $\mathrm{C}(2)-\mathrm{Th}(1)-\mathrm{C}(5)$ & $48.0(3)$ \\
\hline $\mathrm{C}(4) \# 1-\mathrm{Th}(1)-\mathrm{C}(5)$ & $97.3(3)$ \\
\hline $\mathrm{C}(4)-\mathrm{Th}(1)-\mathrm{C}(5)$ & $29.4(3)$ \\
\hline $\mathrm{C}(5) \# 1-\mathrm{Th}(1)-\mathrm{C}(5)$ & $115.7(4)$ \\
\hline $\mathrm{N}(1) \# 1-\mathrm{Th}(1)-\mathrm{C}(1) \# 1$ & $105.6(3)$ \\
\hline $\mathrm{N}(1)-\mathrm{Th}(1)-\mathrm{C}(1) \# 1$ & $79.2(3)$ \\
\hline $\mathrm{C}(3)-\mathrm{Th}(1)-\mathrm{C}(1) \# 1$ & $128.7(3)$ \\
\hline $\mathrm{C}(3) \# 1-\mathrm{Th}(1)-\mathrm{C}(1) \# 1$ & $48.0(3)$ \\
\hline $\mathrm{C}(2) \# 1-\mathrm{Th}(1)-\mathrm{C}(1) \# 1$ & $29.0(3)$ \\
\hline $\mathrm{C}(2)-\mathrm{Th}(1)-\mathrm{C}(1) \# 1$ & $152.0(3)$ \\
\hline $\mathrm{C}(4) \# 1-\mathrm{Th}(1)-\mathrm{C}(1) \# 1$ & $47.8(3)$ \\
\hline $\mathrm{C}(4)-\mathrm{Th}(1)-\mathrm{C}(1) \# 1$ & $125.4(3)$ \\
\hline $\mathrm{C}(5) \# 1-\mathrm{Th}(1)-\mathrm{C}(1) \# 1$ & $28.9(3)$ \\
\hline $\mathrm{C}(5)-\mathrm{Th}(1)-\mathrm{C}(1) \# 1$ & $143.6(3)$ \\
\hline $\mathrm{N}(1) \# 1-\mathrm{Th}(1)-\mathrm{C}(1)$ & $79.2(3)$ \\
\hline $\mathrm{N}(1)-\mathrm{Th}(1)-\mathrm{C}(1)$ & $105.6(3)$ \\
\hline $\mathrm{C}(3)-\mathrm{Th}(1)-\mathrm{C}(1)$ & $48.0(3)$ \\
\hline $\mathrm{C}(3) \# 1-\mathrm{Th}(1)-\mathrm{C}(1)$ & $128.7(3)$ \\
\hline $\mathrm{C}(2) \# 1-\mathrm{Th}(1)-\mathrm{C}(1)$ & $152.0(3)$ \\
\hline $\mathrm{C}(2)-\mathrm{Th}(1)-\mathrm{C}(1)$ & $29.0(3)$ \\
\hline $\mathrm{C}(4) \# 1-\mathrm{Th}(1)-\mathrm{C}(1)$ & $125.4(3)$ \\
\hline $\mathrm{C}(4)-\mathrm{Th}(1)-\mathrm{C}(1)$ & $47.8(3)$ \\
\hline $\mathrm{C}(5) \# 1-\mathrm{Th}(1)-\mathrm{C}(1)$ & $143.6(3)$ \\
\hline $\mathrm{C}(5)-\mathrm{Th}(1)-\mathrm{C}(1)$ & $28.9(3)$ \\
\hline $\mathrm{C}(1) \# 1-\mathrm{Th}(1)-\mathrm{C}(1)$ & $172.4(4)$ \\
\hline
\end{tabular}




\begin{tabular}{|c|c|}
\hline$C(5)-C(1)-C(2)$ & $108.4(9)$ \\
\hline$C(5)-C(1)-C(6)$ & $124.8(9)$ \\
\hline$C(2)-C(1)-C(6)$ & $126.6(10)$ \\
\hline $\mathrm{C}(5)-\mathrm{C}(1)-\mathrm{Th}(1)$ & $74.6(6)$ \\
\hline $\mathrm{C}(2)-\mathrm{C}(1)-\mathrm{Th}(1)$ & $74.3(6)$ \\
\hline $\mathrm{C}(6)-\mathrm{C}(1)-\mathrm{Th}(1)$ & $121.6(7)$ \\
\hline $\mathrm{C}(1)-\mathrm{C}(2)-\mathrm{C}(3)$ & $108.4(9)$ \\
\hline $\mathrm{C}(1)-\mathrm{C}(2)-\mathrm{C}(7)$ & $125.9(11)$ \\
\hline $\mathrm{C}(3)-\mathrm{C}(2)-\mathrm{C}(7)$ & $125.3(10)$ \\
\hline $\mathrm{C}(1)-\mathrm{C}(2)-\mathrm{Th}(1)$ & $76.7(6)$ \\
\hline $\mathrm{C}(3)-\mathrm{C}(2)-\mathrm{Th}(1)$ & $75.0(6)$ \\
\hline $\mathrm{C}(7)-\mathrm{C}(2)-\mathrm{Th}(1)$ & $120.1(7)$ \\
\hline $\mathrm{C}(4)-\mathrm{C}(3)-\mathrm{C}(2)$ & 107.6(9) \\
\hline $\mathrm{C}(4)-\mathrm{C}(3)-\mathrm{C}(8)$ & $126.1(11)$ \\
\hline $\mathrm{C}(2)-\mathrm{C}(3)-\mathrm{C}(8)$ & $126.3(11)$ \\
\hline $\mathrm{C}(4)-\mathrm{C}(3)-\mathrm{Th}(1)$ & $75.7(6)$ \\
\hline $\mathrm{C}(2)-\mathrm{C}(3)-\mathrm{Th}(1)$ & $75.8(6)$ \\
\hline $\mathrm{C}(8)-\mathrm{C}(3)-\mathrm{Th}(1)$ & $116.0(7)$ \\
\hline$C(3)-C(4)-C(5)$ & $108.0(9)$ \\
\hline $\mathrm{C}(3)-\mathrm{C}(4)-\mathrm{C}(9)$ & $125.1(10)$ \\
\hline $\mathrm{C}(5)-\mathrm{C}(4)-\mathrm{C}(9)$ & $126.3(10)$ \\
\hline $\mathrm{C}(3)-\mathrm{C}(4)-\mathrm{Th}(1)$ & $75.0(6)$ \\
\hline $\mathrm{C}(5)-\mathrm{C}(4)-\mathrm{Th}(1)$ & $75.6(5)$ \\
\hline $\mathrm{C}(9)-\mathrm{C}(4)-\mathrm{Th}(1)$ & $122.1(7)$ \\
\hline $\mathrm{C}(1)-\mathrm{C}(5)-\mathrm{C}(4)$ & $107.6(9)$ \\
\hline $\mathrm{C}(1)-\mathrm{C}(5)-\mathrm{C}(10)$ & $126.7(10)$ \\
\hline $\mathrm{C}(4)-\mathrm{C}(5)-\mathrm{C}(10)$ & $125.7(9)$ \\
\hline $\mathrm{C}(1)-\mathrm{C}(5)-\mathrm{Th}(1)$ & $76.5(6)$ \\
\hline $\mathrm{C}(4)-\mathrm{C}(5)-\mathrm{Th}(1)$ & $75.0(6)$ \\
\hline$C(10)-C(5)-\operatorname{Th}(1)$ & $117.0(7)$ \\
\hline $\mathrm{N}(1)-\mathrm{C}(11)-\mathrm{C}(12)$ & $123.4(10)$ \\
\hline N(1)-C(11)-C(18) & $120.5(9)$ \\
\hline $\mathrm{C}(12)-\mathrm{C}(11)-\mathrm{C}(18)$ & $116.1(9)$ \\
\hline$C(13)-C(12)-C(17)$ & $117.2(10)$ \\
\hline$C(13)-C(12)-C(11)$ & $124.0(11)$ \\
\hline$C(17)-C(12)-C(11)$ & $118.7(9)$ \\
\hline
\end{tabular}




$\begin{array}{ll}\mathrm{C}(14)-\mathrm{C}(13)-\mathrm{C}(12) & 121.7(13) \\ \mathrm{C}(13)-\mathrm{C}(14)-\mathrm{C}(15) & 119.6(12) \\ \mathrm{C}(16)-\mathrm{C}(15)-\mathrm{C}(14) & 120.0(12) \\ \mathrm{C}(15)-\mathrm{C}(16)-\mathrm{C}(17) & 122.0(13) \\ \mathrm{C}(16)-\mathrm{C}(17)-\mathrm{C}(12) & 119.4(12) \\ \mathrm{C}(19)-\mathrm{C}(18)-\mathrm{C}(11) & 115.7(9) \\ \mathrm{C}(24)-\mathrm{C}(19)-\mathrm{C}(20) & 117.2(11) \\ \mathrm{C}(24)-\mathrm{C}(19)-\mathrm{C}(18) & 123.4(10) \\ \mathrm{C}(20)-\mathrm{C}(19)-\mathrm{C}(18) & 119.1(10) \\ \mathrm{C}(19)-\mathrm{C}(20)-\mathrm{C}(21) & 119.1(13) \\ \mathrm{C}(22)-\mathrm{C}(21)-\mathrm{C}(20) & 121.4(13) \\ \mathrm{C}(21)-\mathrm{C}(22)-\mathrm{C}(23) & 119.3(12) \\ \mathrm{C}(22)-\mathrm{C}(23)-\mathrm{C}(24) & 120.2(12) \\ \mathrm{C}(23)-\mathrm{C}(24)-\mathrm{C}(19) & 122.5(11)\end{array}$

Symmetry transformations used to generate equivalent atoms:

$\# 1-\mathrm{x}-1, \mathrm{y},-\mathrm{z}+1 / 2$ 
Table S19. Anisotropic displacement parameters $\left(\AA^{2} \mathrm{x} 10^{3}\right)$ for $\left(\mathrm{C}_{5} \mathrm{Me}_{5}\right)_{2} \mathrm{Th}[-\mathrm{N}=\mathrm{C}(\mathrm{Ph})(\mathrm{Bz})]_{2}(\mathbf{1 0 b})$. The anisotropic displacement factor exponent takes the form: $-2 \pi^{2}\left[h^{2} a^{* 2} U^{11}+\ldots+2 h k a^{*} b^{*} U^{12}\right]$

\begin{tabular}{|c|c|c|c|c|c|c|}
\hline & $\mathrm{U}^{11}$ & $\mathrm{U}^{22}$ & $\mathrm{U}^{33}$ & $\mathrm{U}^{23}$ & $\mathrm{U}^{13}$ & $\mathrm{U}^{12}$ \\
\hline $\mathrm{N}(1)$ & $45(5)$ & $49(5)$ & $35(5)$ & $12(5)$ & $5(4)$ & $1(4)$ \\
\hline $\operatorname{Th}(1)$ & $31(1)$ & $35(1)$ & $27(1)$ & 0 & $4(1)$ & 0 \\
\hline$C(1)$ & $40(6)$ & $52(7)$ & $32(6)$ & $11(5)$ & $7(5)$ & $1(5)$ \\
\hline$C(2)$ & $35(6)$ & $58(7)$ & $43(7)$ & $12(6)$ & $17(5)$ & $3(5)$ \\
\hline$C(3)$ & $38(6)$ & $61(8)$ & $39(7)$ & $4(6)$ & $2(5)$ & $-4(5)$ \\
\hline$C(4)$ & $29(5)$ & $48(7)$ & $53(8)$ & $6(5)$ & $3(5)$ & $-6(5)$ \\
\hline$C(5)$ & $34(5)$ & $36(6)$ & $41(7)$ & $6(5)$ & $6(5)$ & $-1(5)$ \\
\hline$C(6)$ & $60(7)$ & $58(7)$ & $44(8)$ & $-10(6)$ & $20(6)$ & $5(6)$ \\
\hline$C(7)$ & $57(7)$ & $64(8)$ & $74(9)$ & $24(7)$ & $35(7)$ & $21(6)$ \\
\hline $\mathrm{C}(8)$ & $47(7)$ & $106(11)$ & $47(8)$ & $23(8)$ & $-6(6)$ & $-22(7)$ \\
\hline$C(9)$ & 78(9) & $64(8)$ & $80(11)$ & $-10(8)$ & $20(8)$ & $-38(7)$ \\
\hline$C(10)$ & $59(7)$ & $62(7)$ & $53(8)$ & $8(7)$ & $3(6)$ & $3(7)$ \\
\hline $\mathrm{C}(11)$ & $42(6)$ & $48(7)$ & $34(6)$ & $-4(5)$ & $2(5)$ & $0(5)$ \\
\hline$C(12)$ & $42(6)$ & $52(7)$ & $35(6)$ & $9(6)$ & $14(5)$ & $0(5)$ \\
\hline$C(13)$ & $54(7)$ & $61(8)$ & $63(9)$ & $5(6)$ & 11(7) & $15(6)$ \\
\hline$C(14)$ & $49(8)$ & $95(11)$ & $62(10)$ & 19(9) & $-7(7)$ & $18(7)$ \\
\hline$C(15)$ & $56(8)$ & $106(11)$ & $47(8)$ & $13(9)$ & $-3(6)$ & $-9(8)$ \\
\hline$C(16)$ & $69(9)$ & $84(10)$ & $47(9)$ & $-3(8)$ & $16(7)$ & $-24(8)$ \\
\hline $\mathrm{C}(17)$ & $45(7)$ & $67(8)$ & $44(8)$ & $-2(6)$ & $4(6)$ & $-1(6)$ \\
\hline$C(18)$ & $62(7)$ & $44(6)$ & $44(7)$ & $0(6)$ & $14(6)$ & $10(6)$ \\
\hline$C(19)$ & $52(7)$ & $41(7)$ & $34(7)$ & $-4(5)$ & $-4(5)$ & $4(5)$ \\
\hline$C(20)$ & $64(8)$ & $49(8)$ & $89(11)$ & $-3(7)$ & $16(8)$ & $5(7)$ \\
\hline$C(21)$ & $94(12)$ & $44(8)$ & $112(14)$ & $9(8)$ & $-2(10)$ & $1(7)$ \\
\hline$C(22)$ & $62(8)$ & $70(9)$ & $63(9)$ & $24(8)$ & $-5(7)$ & $-18(8)$ \\
\hline$C(23)$ & $60(8)$ & $66(9)$ & $56(9)$ & $-4(7)$ & $8(6)$ & $-7(7)$ \\
\hline$C(24)$ & $64(8)$ & $52(7)$ & $55(8)$ & $-13(7)$ & $12(6)$ & $3(7)$ \\
\hline
\end{tabular}


Table S20. Hydrogen coordinates ( x 104) and isotropic displacement parameters $\left(\AA^{2} \times 10^{3}\right)$ for $\left(\mathrm{C}_{5} \mathrm{Me}_{5}\right)_{2} \mathrm{Th}[-\mathrm{N}=\mathrm{C}(\mathrm{Ph})(\mathrm{Bz})]_{2}(\mathbf{1 0 b})$.

\begin{tabular}{|c|c|c|c|c|}
\hline & $\mathrm{x}$ & $\mathrm{y}$ & z & $\mathrm{U}(\mathrm{eq})$ \\
\hline $\mathrm{H}(6 \mathrm{~A})$ & -4767 & 1536 & 381 & 80 \\
\hline $\mathrm{H}(6 \mathrm{~B})$ & -4528 & 552 & 786 & 80 \\
\hline $\mathrm{H}(6 \mathrm{C})$ & -3820 & 1106 & 394 & 80 \\
\hline $\mathrm{H}(7 \mathrm{~A})$ & -3122 & 119 & 1518 & 95 \\
\hline $\mathrm{H}(7 \mathrm{~B})$ & -2983 & 235 & 2325 & 95 \\
\hline $\mathrm{H}(7 \mathrm{C})$ & -2314 & 704 & 1852 & 95 \\
\hline $\mathrm{H}(8 \mathrm{~A})$ & -2741 & 3005 & 3162 & 101 \\
\hline $\mathrm{H}(8 \mathrm{~B})$ & -2115 & 2268 & 2819 & 101 \\
\hline $\mathrm{H}(8 \mathrm{C})$ & -2842 & 1856 & 3263 & 101 \\
\hline $\mathrm{H}(9 \mathrm{~A})$ & -3494 & 4174 & 2743 & 110 \\
\hline $\mathrm{H}(9 \mathrm{~B})$ & -4443 & 4335 & 2408 & 110 \\
\hline $\mathrm{H}(9 \mathrm{C})$ & -3646 & 4584 & 1985 & 110 \\
\hline $\mathrm{H}(10 \mathrm{~A})$ & -5209 & 2948 & 593 & 87 \\
\hline $\mathrm{H}(10 \mathrm{~B})$ & -4702 & 3923 & 809 & 87 \\
\hline $\mathrm{H}(10 \mathrm{C})$ & -5452 & 3550 & 1243 & 87 \\
\hline $\mathrm{H}(13)$ & -2652 & -1498 & 4053 & 70 \\
\hline $\mathrm{H}(14)$ & -1674 & -1368 & 5007 & 83 \\
\hline $\mathrm{H}(15)$ & -1533 & 122 & 5606 & 84 \\
\hline $\mathrm{H}(16)$ & -2400 & 1417 & 5285 & 79 \\
\hline $\mathrm{H}(17)$ & -3416 & 1319 & 4350 & 62 \\
\hline $\mathrm{H}(18 \mathrm{~A})$ & -4166 & -1030 & 2615 & 60 \\
\hline $\mathrm{H}(18 \mathrm{~B})$ & -3321 & -1416 & 3031 & 60 \\
\hline $\mathrm{H}(20)$ & -3809 & -3106 & 3064 & 80 \\
\hline $\mathrm{H}(21)$ & -4626 & -4295 & 3603 & 101 \\
\hline $\mathrm{H}(22)$ & -5840 & -3849 & 4119 & 79 \\
\hline $\mathrm{H}(23)$ & -6117 & -2205 & 4294 & 73 \\
\hline $\mathrm{H}(24)$ & -5272 & -1015 & 3846 & 68 \\
\hline
\end{tabular}


Table S21. Crystal data and structure refinement for $\left(\mathrm{C}_{5} \mathrm{Me}_{5}\right)_{2} \mathrm{U}\left(\mathrm{CH}_{2} \mathrm{Ph}\right)_{2}(\mathbf{1 1 b})$.

Identification code

Empirical formula

Formula weight

Temperature

Wavelength

Crystal system

Space group

Unit cell dimensions

Volume

$\mathrm{Z}$

Density (calculated)

Absorption coefficient

$\mathrm{F}(000)$

Crystal size

Theta range for data collection

Index ranges

Reflections collected

Independent reflections

Max. and min. transmission

Refinement method

Data / restraints / parameters

Goodness-of-fit on $\mathrm{F}^{2}$

Final R indices [I $>2 \operatorname{sigma}(\mathrm{I})]$

$\mathrm{R}$ indices (all data)

Largest diff. peak and hole $\operatorname{ccd} 813$

$\mathrm{C}_{34} \mathrm{H}_{44} \mathrm{U}$

690.72

203(2) K

$0.71073 \AA$

monoclinic

P $2{ }_{1} / \mathrm{n}$

$\mathrm{a}=9.772(3) \AA$

$\alpha=90^{\circ}$.

$\mathrm{b}=12.328(4) \AA$

$\beta=100.514(5)^{\circ}$.

$\mathrm{c}=24.037(7) \AA$

$\gamma=90^{\circ}$.

2847.2(15) $\AA^{3}$

4

$1.611 \mathrm{Mg} / \mathrm{m}^{3}$

$5.720 \mathrm{~mm}^{-1}$

1360

$0.14 \times 0.10 \times 0.06 \mathrm{~mm}^{3}$

1.72 to $25.45^{\circ}$.

$-11<=\mathrm{h}<=11,-14<=\mathrm{k}<=12,-28<=1<=28$

14577

$4871[\mathrm{R}(\mathrm{int})=0.0190]$

0.7253 and 0.5014

Full-matrix least-squares on $\mathrm{F}^{2}$

4871 / 0 / 316

2.181

$\mathrm{R} 1=0.0280, \mathrm{wR} 2=0.0786$

$\mathrm{R} 1=0.0340, \mathrm{wR} 2=0.0798$

1.238 and -1.613 e. $\AA^{-3}$ 
Table S22. Atomic coordinates ( x 104) and equivalent isotropic displacement parameters $\left(\AA^{2} \times 10^{3}\right)$ for $\left(\mathrm{C}_{5} \mathrm{Me}_{5}\right)_{2} \mathrm{U}\left(\mathrm{CH}_{2} \mathrm{Ph}\right)_{2}(\mathbf{1 1 b})$. U(eq) is defined as one third of the trace of the orthogonalized $\mathrm{U}^{\mathrm{ij}}$ tensor.

\begin{tabular}{|c|c|c|c|c|}
\hline & $\mathrm{x}$ & $\mathrm{y}$ & z & $\mathrm{U}(\mathrm{eq})$ \\
\hline $\mathrm{U}(1)$ & 3073(1) & $2314(1)$ & $1142(1)$ & $22(1)$ \\
\hline$C(1)$ & $5116(6)$ & $3142(5)$ & $583(2)$ & $34(1)$ \\
\hline$C(2)$ & $5790(6)$ & $3041(5)$ & $1154(2)$ & $33(1)$ \\
\hline$C(3)$ & $5182(5)$ & $3782(4)$ & 1483(2) & $30(1)$ \\
\hline$C(4)$ & 4147(6) & 4371(4) & $1116(2)$ & $28(1)$ \\
\hline$C(5)$ & $4082(6)$ & $3957(4)$ & $558(2)$ & $31(1)$ \\
\hline$C(6)$ & 5592(9) & $2694(5)$ & 72(3) & $53(2)$ \\
\hline$C(7)$ & 7077(7) & $2382(5)$ & 1375(3) & $49(2)$ \\
\hline $\mathrm{C}(8)$ & 5694(6) & 4053(5) & 2093(2) & $39(2)$ \\
\hline$C(9)$ & $3519(7)$ & $5408(5)$ & 1288(3) & $43(2)$ \\
\hline $\mathrm{C}(10)$ & $3185(7)$ & $4378(5)$ & $24(2)$ & $45(2)$ \\
\hline$C(11)$ & $663(6)$ & 1948(5) & $1590(2)$ & $30(1)$ \\
\hline $\mathrm{C}(12)$ & $873(6)$ & $3076(5)$ & 1597(2) & $30(1)$ \\
\hline $\mathrm{C}(13)$ & $590(5)$ & $3454(4)$ & $1025(2)$ & $28(1)$ \\
\hline$C(14)$ & $257(6)$ & $2542(4)$ & $672(2)$ & $28(1)$ \\
\hline$C(15)$ & $287(5)$ & $1617(5)$ & $1018(2)$ & $30(1)$ \\
\hline$C(16)$ & $567(7)$ & $1256(6)$ & 2093(3) & $52(2)$ \\
\hline$C(17)$ & $1117(7)$ & $3787(6)$ & $2110(2)$ & $48(2)$ \\
\hline $\mathrm{C}(18)$ & $295(6)$ & $4617(5)$ & $844(3)$ & $40(2)$ \\
\hline$C(19)$ & $-243(7)$ & $2584(5)$ & $42(2)$ & $42(2)$ \\
\hline$C(20)$ & $-324(7)$ & $525(5)$ & $815(3)$ & $50(2)$ \\
\hline$C(21)$ & $2920(6)$ & 1061(4) & $321(2)$ & $32(1)$ \\
\hline $\mathrm{C}(22)$ & $3687(6)$ & $189(4)$ & $660(2)$ & $31(1)$ \\
\hline$C(23)$ & $3014(6)$ & $-583(5)$ & $938(2)$ & $37(1)$ \\
\hline $\mathrm{C}(24)$ & $3744(7)$ & $-1345(5)$ & $1302(3)$ & $44(2)$ \\
\hline$C(25)$ & $5153(7)$ & $-1356(5)$ & $1400(3)$ & $45(2)$ \\
\hline$C(26)$ & $5840(7)$ & $-625(5)$ & $1122(3)$ & $44(2)$ \\
\hline$C(27)$ & $5133(6)$ & $132(5)$ & $757(2)$ & $37(1)$ \\
\hline$C(28)$ & $4094(6)$ & 1291(4) & $2000(2)$ & $30(1)$ \\
\hline$C(29)$ & $4843(6)$ & $1600(4)$ & $2569(2)$ & $27(1)$ \\
\hline $\mathrm{C}(30)$ & $6247(6)$ & $1312(5)$ & $2759(2)$ & $37(1)$ \\
\hline
\end{tabular}




\begin{tabular}{lllll}
$\mathrm{C}(31)$ & $6950(7)$ & $1621(5)$ & $3286(3)$ & $46(2)$ \\
$\mathrm{C}(32)$ & $6310(8)$ & $2212(5)$ & $3645(3)$ & $46(2)$ \\
$\mathrm{C}(33)$ & $4938(8)$ & $2486(5)$ & $3475(3)$ & $44(2)$ \\
$\mathrm{C}(34)$ & $4220(7)$ & $2186(4)$ & $2951(2)$ & $35(1)$ \\
\hline
\end{tabular}


Table S23. Bond lengths $[\AA]$ and angles $\left[^{\circ}\right]$ for $\left(\mathrm{C}_{5} \mathrm{Me}_{5}\right)_{2} \mathrm{U}\left(\mathrm{CH}_{2} \mathrm{Ph}\right)_{2}(\mathbf{1 1 b})$.

\begin{tabular}{|c|c|}
\hline $\mathrm{U}(1)-\mathrm{C}(28)$ & $2.467(5)$ \\
\hline $\mathrm{U}(1)-\mathrm{C}(21)$ & $2.489(5)$ \\
\hline $\mathrm{U}(1)-\mathrm{C}(4)$ & $2.749(5)$ \\
\hline $\mathrm{U}(1)-\mathrm{C}(5)$ & $2.747(5)$ \\
\hline $\mathrm{U}(1)-\mathrm{C}(12)$ & $2.750(6)$ \\
\hline $\mathrm{U}(1)-\mathrm{C}(3)$ & $2.752(5)$ \\
\hline $\mathrm{U}(1)-\mathrm{C}(13)$ & $2.774(5)$ \\
\hline $\mathrm{U}(1)-\mathrm{C}(14)$ & $2.792(6)$ \\
\hline $\mathrm{U}(1)-\mathrm{C}(1)$ & $2.794(6)$ \\
\hline $\mathrm{U}(1)-\mathrm{C}(2)$ & $2.798(6)$ \\
\hline $\mathrm{U}(1)-\mathrm{C}(11)$ & $2.800(6)$ \\
\hline $\mathrm{U}(1)-\mathrm{C}(15)$ & $2.819(5)$ \\
\hline $\mathrm{C}(1)-\mathrm{C}(2)$ & $1.415(8)$ \\
\hline$C(1)-C(5)$ & $1.418(8)$ \\
\hline$C(1)-C(6)$ & $1.497(8)$ \\
\hline $\mathrm{C}(2)-\mathrm{C}(3)$ & $1.410(8)$ \\
\hline$C(2)-C(7)$ & $1.510(9)$ \\
\hline$C(3)-C(4)$ & $1.415(7)$ \\
\hline $\mathrm{C}(3)-\mathrm{C}(8)$ & $1.498(7)$ \\
\hline$C(4)-C(5)$ & $1.426(7)$ \\
\hline $\mathrm{C}(4)-\mathrm{C}(9)$ & $1.507(8)$ \\
\hline$C(5)-C(10)$ & $1.508(7)$ \\
\hline $\mathrm{C}(11)-\mathrm{C}(12)$ & $1.405(9)$ \\
\hline$C(11)-C(15)$ & $1.416(7)$ \\
\hline$C(11)-C(16)$ & $1.497(8)$ \\
\hline$C(12)-C(13)$ & $1.432(7)$ \\
\hline$C(12)-C(17)$ & $1.495(7)$ \\
\hline$C(13)-C(14)$ & $1.409(7)$ \\
\hline$C(13)-C(18)$ & $1.510(8)$ \\
\hline$C(14)-C(15)$ & $1.408(8)$ \\
\hline$C(14)-C(19)$ & $1.506(8)$ \\
\hline$C(15)-C(20)$ & $1.518(8)$ \\
\hline$C(21)-C(22)$ & $1.470(8)$ \\
\hline $\mathrm{C}(22)-\mathrm{C}(27)$ & $1.391(8)$ \\
\hline
\end{tabular}




\begin{tabular}{|c|c|}
\hline$C(22)-C(23)$ & $1.395(8)$ \\
\hline$C(23)-C(24)$ & $1.388(8)$ \\
\hline$C(24)-C(25)$ & $1.354(9)$ \\
\hline$C(25)-C(26)$ & $1.369(9)$ \\
\hline$C(26)-C(27)$ & $1.377(8)$ \\
\hline C(28)-C(29) & $1.479(6)$ \\
\hline C(29)-C(34) & $1.394(8)$ \\
\hline$C(29)-C(30)$ & $1.410(7)$ \\
\hline $\mathrm{C}(30)-\mathrm{C}(31)$ & $1.380(7)$ \\
\hline $\mathrm{C}(31)-\mathrm{C}(32)$ & $1.365(10)$ \\
\hline$C(32)-C(33)$ & $1.371(11)$ \\
\hline C(33)-C(34) & $1.375(8)$ \\
\hline $\mathrm{C}(28)-\mathrm{U}(1)-\mathrm{C}(21)$ & $107.55(18)$ \\
\hline $\mathrm{C}(28)-\mathrm{U}(1)-\mathrm{C}(4)$ & $113.13(17)$ \\
\hline $\mathrm{C}(21)-\mathrm{U}(1)-\mathrm{C}(4)$ & $121.51(18)$ \\
\hline $\mathrm{C}(28)-\mathrm{U}(1)-\mathrm{C}(5)$ & $132.52(17)$ \\
\hline $\mathrm{C}(21)-\mathrm{U}(1)-\mathrm{C}(5)$ & $91.60(18)$ \\
\hline $\mathrm{C}(4)-\mathrm{U}(1)-\mathrm{C}(5)$ & $30.07(15)$ \\
\hline $\mathrm{C}(28)-\mathrm{U}(1)-\mathrm{C}(12)$ & $93.92(17)$ \\
\hline $\mathrm{C}(21)-\mathrm{U}(1)-\mathrm{C}(12)$ & $126.08(18)$ \\
\hline $\mathrm{C}(4)-\mathrm{U}(1)-\mathrm{C}(12)$ & $91.35(18)$ \\
\hline $\mathrm{C}(5)-\mathrm{U}(1)-\mathrm{C}(12)$ & $109.35(18)$ \\
\hline $\mathrm{C}(28)-\mathrm{U}(1)-\mathrm{C}(3)$ & $85.45(17)$ \\
\hline $\mathrm{C}(21)-\mathrm{U}(1)-\mathrm{C}(3)$ & $125.31(18)$ \\
\hline $\mathrm{C}(4)-\mathrm{U}(1)-\mathrm{C}(3)$ & $29.82(15)$ \\
\hline $\mathrm{C}(5)-\mathrm{U}(1)-\mathrm{C}(3)$ & $49.37(16)$ \\
\hline $\mathrm{C}(12)-\mathrm{U}(1)-\mathrm{C}(3)$ & $104.73(17)$ \\
\hline $\mathrm{C}(28)-\mathrm{U}(1)-\mathrm{C}(13)$ & $123.86(17)$ \\
\hline $\mathrm{C}(21)-\mathrm{U}(1)-\mathrm{C}(13)$ & 107.98(17) \\
\hline $\mathrm{C}(4)-\mathrm{U}(1)-\mathrm{C}(13)$ & $82.00(16)$ \\
\hline $\mathrm{C}(5)-\mathrm{U}(1)-\mathrm{C}(13)$ & $87.90(17)$ \\
\hline $\mathrm{C}(12)-\mathrm{U}(1)-\mathrm{C}(13)$ & $30.05(15)$ \\
\hline $\mathrm{C}(3)-\mathrm{U}(1)-\mathrm{C}(13)$ & $106.90(16)$ \\
\hline $\mathrm{C}(28)-\mathrm{U}(1)-\mathrm{C}(14)$ & $127.43(18)$ \\
\hline $\mathrm{C}(21)-\mathrm{U}(1)-\mathrm{C}(14)$ & $80.18(17)$ \\
\hline
\end{tabular}




\begin{tabular}{|c|c|}
\hline $\mathrm{C}(4)-\mathrm{U}(1)-\mathrm{C}(14)$ & $104.49(16)$ \\
\hline $\mathrm{C}(5)-\mathrm{U}(1)-\mathrm{C}(14)$ & $97.95(16)$ \\
\hline $\mathrm{C}(12)-\mathrm{U}(1)-\mathrm{C}(14)$ & $48.83(15)$ \\
\hline $\mathrm{C}(3)-\mathrm{U}(1)-\mathrm{C}(14)$ & $133.12(16)$ \\
\hline $\mathrm{C}(13)-\mathrm{U}(1)-\mathrm{C}(14)$ & $29.33(15)$ \\
\hline $\mathrm{C}(28)-\mathrm{U}(1)-\mathrm{C}(1)$ & 111.91(19) \\
\hline $\mathrm{C}(21)-\mathrm{U}(1)-\mathrm{C}(1)$ & 77.91(18) \\
\hline $\mathrm{C}(4)-\mathrm{U}(1)-\mathrm{C}(1)$ & $48.95(17)$ \\
\hline $\mathrm{C}(5)-\mathrm{U}(1)-\mathrm{C}(1)$ & $29.64(18)$ \\
\hline $\mathrm{C}(12)-\mathrm{U}(1)-\mathrm{C}(1)$ & $138.2(2)$ \\
\hline $\mathrm{C}(3)-\mathrm{U}(1)-\mathrm{C}(1)$ & $48.83(16)$ \\
\hline $\mathrm{C}(13)-\mathrm{U}(1)-\mathrm{C}(1)$ & $117.02(18)$ \\
\hline $\mathrm{C}(14)-\mathrm{U}(1)-\mathrm{C}(1)$ & $120.44(17)$ \\
\hline $\mathrm{C}(28)-\mathrm{U}(1)-\mathrm{C}(2)$ & $85.24(18)$ \\
\hline $\mathrm{C}(21)-\mathrm{U}(1)-\mathrm{C}(2)$ & $97.28(18)$ \\
\hline $\mathrm{C}(4)-\mathrm{U}(1)-\mathrm{C}(2)$ & $48.65(17)$ \\
\hline $\mathrm{C}(5)-\mathrm{U}(1)-\mathrm{C}(2)$ & $48.75(17)$ \\
\hline $\mathrm{C}(12)-\mathrm{U}(1)-\mathrm{C}(2)$ & $134.13(18)$ \\
\hline $\mathrm{C}(3)-\mathrm{U}(1)-\mathrm{C}(2)$ & $29.41(16)$ \\
\hline $\mathrm{C}(13)-\mathrm{U}(1)-\mathrm{C}(2)$ & $130.53(17)$ \\
\hline $\mathrm{C}(14)-\mathrm{U}(1)-\mathrm{C}(2)$ & $146.68(17)$ \\
\hline $\mathrm{C}(1)-\mathrm{U}(1)-\mathrm{C}(2)$ & $29.32(16)$ \\
\hline $\mathrm{C}(28)-\mathrm{U}(1)-\mathrm{C}(11)$ & $80.59(17)$ \\
\hline $\mathrm{C}(21)-\mathrm{U}(1)-\mathrm{C}(11)$ & $105.76(18)$ \\
\hline $\mathrm{C}(4)-\mathrm{U}(1)-\mathrm{C}(11)$ & $120.58(17)$ \\
\hline $\mathrm{C}(5)-\mathrm{U}(1)-\mathrm{C}(11)$ & $136.13(17)$ \\
\hline $\mathrm{C}(12)-\mathrm{U}(1)-\mathrm{C}(11)$ & 29.31(19) \\
\hline $\mathrm{C}(3)-\mathrm{U}(1)-\mathrm{C}(11)$ & $128.90(17)$ \\
\hline $\mathrm{C}(13)-\mathrm{U}(1)-\mathrm{C}(11)$ & $48.62(16)$ \\
\hline $\mathrm{C}(14)-\mathrm{U}(1)-\mathrm{C}(11)$ & $48.33(16)$ \\
\hline $\mathrm{C}(1)-\mathrm{U}(1)-\mathrm{C}(11)$ & $165.63(17)$ \\
\hline $\mathrm{C}(2)-\mathrm{U}(1)-\mathrm{C}(11)$ & $155.70(17)$ \\
\hline $\mathrm{C}(28)-\mathrm{U}(1)-\mathrm{C}(15)$ & $99.66(17)$ \\
\hline$C(21)-U(1)-C(15)$ & $79.03(18)$ \\
\hline $\mathrm{C}(4)-\mathrm{U}(1)-\mathrm{C}(15)$ & $130.09(16)$ \\
\hline C(5)-U(1)-C(15) & $126.90(16)$ \\
\hline
\end{tabular}




\begin{tabular}{|c|c|}
\hline $\mathrm{C}(12)-\mathrm{U}(1)-\mathrm{C}(15)$ & $48.36(16)$ \\
\hline $\mathrm{C}(3)-\mathrm{U}(1)-\mathrm{C}(15)$ & $152.63(17)$ \\
\hline $\mathrm{C}(13)-\mathrm{U}(1)-\mathrm{C}(15)$ & $48.19(17)$ \\
\hline $\mathrm{C}(14)-\mathrm{U}(1)-\mathrm{C}(15)$ & $29.07(16)$ \\
\hline C(1)-U(1)-C(15) & $145.23(16)$ \\
\hline $\mathrm{C}(2)-\mathrm{U}(1)-\mathrm{C}(15)$ & $174.55(16)$ \\
\hline $\mathrm{C}(11)-\mathrm{U}(1)-\mathrm{C}(15)$ & $29.20(15)$ \\
\hline $\mathrm{C}(2)-\mathrm{C}(1)-\mathrm{C}(5)$ & $107.8(5)$ \\
\hline $\mathrm{C}(2)-\mathrm{C}(1)-\mathrm{C}(6)$ & $127.1(6)$ \\
\hline$C(5)-C(1)-C(6)$ & $123.5(6)$ \\
\hline $\mathrm{C}(2)-\mathrm{C}(1)-\mathrm{U}(1)$ & $75.5(3)$ \\
\hline $\mathrm{C}(5)-\mathrm{C}(1)-\mathrm{U}(1)$ & 73.3(3) \\
\hline $\mathrm{C}(6)-\mathrm{C}(1)-\mathrm{U}(1)$ & $128.1(4)$ \\
\hline $\mathrm{C}(3)-\mathrm{C}(2)-\mathrm{C}(1)$ & $108.5(5)$ \\
\hline $\mathrm{C}(3)-\mathrm{C}(2)-\mathrm{C}(7)$ & $124.0(5)$ \\
\hline $\mathrm{C}(1)-\mathrm{C}(2)-\mathrm{C}(7)$ & $126.9(6)$ \\
\hline $\mathrm{C}(3)-\mathrm{C}(2)-\mathrm{U}(1)$ & $73.5(3)$ \\
\hline $\mathrm{C}(1)-\mathrm{C}(2)-\mathrm{U}(1)$ & $75.2(3)$ \\
\hline $\mathrm{C}(7)-\mathrm{C}(2)-\mathrm{U}(1)$ & $124.0(4)$ \\
\hline $\mathrm{C}(2)-\mathrm{C}(3)-\mathrm{C}(4)$ & $108.0(5)$ \\
\hline $\mathrm{C}(2)-\mathrm{C}(3)-\mathrm{C}(8)$ & $126.5(5)$ \\
\hline $\mathrm{C}(4)-\mathrm{C}(3)-\mathrm{C}(8)$ & $124.7(5)$ \\
\hline $\mathrm{C}(2)-\mathrm{C}(3)-\mathrm{U}(1)$ & 77.1(3) \\
\hline $\mathrm{C}(4)-\mathrm{C}(3)-\mathrm{U}(1)$ & $75.0(3)$ \\
\hline $\mathrm{C}(8)-\mathrm{C}(3)-\mathrm{U}(1)$ & $122.4(4)$ \\
\hline$C(3)-C(4)-C(5)$ & $107.9(5)$ \\
\hline $\mathrm{C}(3)-\mathrm{C}(4)-\mathrm{C}(9)$ & $123.0(5)$ \\
\hline$C(5)-C(4)-C(9)$ & $127.6(5)$ \\
\hline $\mathrm{C}(3)-\mathrm{C}(4)-\mathrm{U}(1)$ & $75.2(3)$ \\
\hline $\mathrm{C}(5)-\mathrm{C}(4)-\mathrm{U}(1)$ & $74.9(3)$ \\
\hline $\mathrm{C}(9)-\mathrm{C}(4)-\mathrm{U}(1)$ & $126.9(4)$ \\
\hline$C(1)-C(5)-C(4)$ & $107.7(5)$ \\
\hline $\mathrm{C}(1)-\mathrm{C}(5)-\mathrm{C}(10)$ & $125.4(5)$ \\
\hline $\mathrm{C}(4)-\mathrm{C}(5)-\mathrm{C}(10)$ & $126.6(5)$ \\
\hline $\mathrm{C}(1)-\mathrm{C}(5)-\mathrm{U}(1)$ & $77.0(3)$ \\
\hline $\mathrm{C}(4)-\mathrm{C}(5)-\mathrm{U}(1)$ & $75.0(3)$ \\
\hline
\end{tabular}




\begin{tabular}{|c|c|}
\hline $\mathrm{C}(10)-\mathrm{C}(5)-\mathrm{U}(1)$ & $118.8(4)$ \\
\hline $\mathrm{C}(12)-\mathrm{C}(11)-\mathrm{C}(15)$ & $108.0(4)$ \\
\hline$C(12)-C(11)-C(16)$ & $125.8(5)$ \\
\hline$C(15)-C(11)-C(16)$ & $125.2(6)$ \\
\hline $\mathrm{C}(12)-\mathrm{C}(11)-\mathrm{U}(1)$ & $73.4(3)$ \\
\hline $\mathrm{C}(15)-\mathrm{C}(11)-\mathrm{U}(1)$ & $76.1(3)$ \\
\hline$C(16)-C(11)-U(1)$ & $125.7(4)$ \\
\hline$C(11)-C(12)-C(13)$ & 107.9(4) \\
\hline $\mathrm{C}(11)-\mathrm{C}(12)-\mathrm{C}(17)$ & $126.3(5)$ \\
\hline$C(13)-C(12)-C(17)$ & $125.1(5)$ \\
\hline $\mathrm{C}(11)-\mathrm{C}(12)-\mathrm{U}(1)$ & $77.3(3)$ \\
\hline $\mathrm{C}(13)-\mathrm{C}(12)-\mathrm{U}(1)$ & $75.9(3)$ \\
\hline $\mathrm{C}(17)-\mathrm{C}(12)-\mathrm{U}(1)$ & $120.6(4)$ \\
\hline $\mathrm{C}(14)-\mathrm{C}(13)-\mathrm{C}(12)$ & $107.5(5)$ \\
\hline$C(14)-C(13)-C(18)$ & $125.0(5)$ \\
\hline $\mathrm{C}(12)-\mathrm{C}(13)-\mathrm{C}(18)$ & $125.3(5)$ \\
\hline $\mathrm{C}(14)-\mathrm{C}(13)-\mathrm{U}(1)$ & $76.1(3)$ \\
\hline $\mathrm{C}(12)-\mathrm{C}(13)-\mathrm{U}(1)$ & $74.1(3)$ \\
\hline $\mathrm{C}(18)-\mathrm{C}(13)-\mathrm{U}(1)$ & $128.9(4)$ \\
\hline$C(15)-C(14)-C(13)$ & $108.3(5)$ \\
\hline$C(15)-C(14)-C(19)$ & $126.1(5)$ \\
\hline$C(13)-C(14)-C(19)$ & $125.1(5)$ \\
\hline $\mathrm{C}(15)-\mathrm{C}(14)-\mathrm{U}(1)$ & $76.5(3)$ \\
\hline $\mathrm{C}(13)-\mathrm{C}(14)-\mathrm{U}(1)$ & $74.6(3)$ \\
\hline$C(19)-C(14)-U(1)$ & $121.6(4)$ \\
\hline$C(14)-C(15)-C(11)$ & $108.3(5)$ \\
\hline$C(14)-C(15)-C(20)$ & $124.3(5)$ \\
\hline $\mathrm{C}(11)-\mathrm{C}(15)-\mathrm{C}(20)$ & $125.8(5)$ \\
\hline $\mathrm{C}(14)-\mathrm{C}(15)-\mathrm{U}(1)$ & $74.4(3)$ \\
\hline $\mathrm{C}(11)-\mathrm{C}(15)-\mathrm{U}(1)$ & $74.7(3)$ \\
\hline$C(20)-C(15)-U(1)$ & $128.3(4)$ \\
\hline$C(22)-C(21)-U(1)$ & $93.6(3)$ \\
\hline $\mathrm{C}(27)-\mathrm{C}(22)-\mathrm{C}(23)$ & $116.0(5)$ \\
\hline$C(27)-C(22)-C(21)$ & $121.9(5)$ \\
\hline$C(23)-C(22)-C(21)$ & $121.9(5)$ \\
\hline $\mathrm{C}(27)-\mathrm{C}(22)-\mathrm{U}(1)$ & $104.5(3)$ \\
\hline
\end{tabular}




$\begin{array}{lr}\mathrm{C}(23)-\mathrm{C}(22)-\mathrm{U}(1) & 105.6(4) \\ \mathrm{C}(21)-\mathrm{C}(22)-\mathrm{U}(1) & 56.8(3) \\ \mathrm{C}(24)-\mathrm{C}(23)-\mathrm{C}(22) & 122.0(6) \\ \mathrm{C}(25)-\mathrm{C}(24)-\mathrm{C}(23) & 120.4(6) \\ \mathrm{C}(24)-\mathrm{C}(25)-\mathrm{C}(26) & 118.8(6) \\ \mathrm{C}(25)-\mathrm{C}(26)-\mathrm{C}(27) & 121.6(6) \\ \mathrm{C}(26)-\mathrm{C}(27)-\mathrm{C}(22) & 121.1(6) \\ \mathrm{C}(29)-\mathrm{C}(28)-\mathrm{U}(1) & 134.2(4) \\ \mathrm{C}(34)-\mathrm{C}(29)-\mathrm{C}(30) & 115.7(5) \\ \mathrm{C}(34)-\mathrm{C}(29)-\mathrm{C}(28) & 122.8(5) \\ \mathrm{C}(30)-\mathrm{C}(29)-\mathrm{C}(28) & 121.5(5) \\ \mathrm{C}(31)-\mathrm{C}(30)-\mathrm{C}(29) & 121.3(6) \\ \mathrm{C}(32)-\mathrm{C}(31)-\mathrm{C}(30) & 121.2(6) \\ \mathrm{C}(31)-\mathrm{C}(32)-\mathrm{C}(33) & 118.7(5) \\ \mathrm{C}(32)-\mathrm{C}(33)-\mathrm{C}(34) & 120.9(6) \\ \mathrm{C}(33)-\mathrm{C}(34)-\mathrm{C}(29) & 122.2(6)\end{array}$

Symmetry transformations used to generate equivalent atoms: 
Table S24. Anisotropic displacement parameters $\left(\AA^{2} \times 10^{3}\right)$ for $\left(\mathrm{C}_{5} \mathrm{Me}_{5}\right)_{2} \mathrm{U}\left(\mathrm{CH}_{2} \mathrm{Ph}\right)_{2}$ (11b). The anisotropic displacement factor exponent takes the form: $-2 \pi^{2}\left[\mathrm{~h}^{2} \mathrm{a}^{* 2} \mathrm{U}^{11}+\ldots+2 \mathrm{hk} \mathrm{a}^{*} \mathrm{~b}^{*} \mathrm{U}^{12}\right]$

\begin{tabular}{|c|c|c|c|c|c|c|}
\hline & $\mathrm{U}^{11}$ & $U^{22}$ & $\mathrm{U}^{33}$ & $\mathrm{U}^{23}$ & $\mathrm{U}^{13}$ & $\mathrm{U}^{12}$ \\
\hline $\mathrm{U}(1)$ & 21(1) & $23(1)$ & $22(1)$ & $-2(1)$ & $6(1)$ & $-1(1)$ \\
\hline$C(1)$ & $33(4)$ & $34(3)$ & $42(3)$ & $-9(2)$ & $23(3)$ & $-10(3)$ \\
\hline$C(2)$ & $23(3)$ & $28(3)$ & $52(3)$ & $-3(3)$ & $16(3)$ & $-10(2)$ \\
\hline$C(3)$ & $20(3)$ & $26(3)$ & $45(3)$ & $-3(2)$ & $6(2)$ & $-7(2)$ \\
\hline$C(4)$ & $23(3)$ & $20(3)$ & $42(3)$ & $-2(2)$ & 11(2) & $-6(2)$ \\
\hline$C(5)$ & $36(4)$ & $30(3)$ & 27(3) & $-1(2)$ & $9(2)$ & $-12(3)$ \\
\hline$C(6)$ & $68(5)$ & 47(4) & $56(4)$ & $-14(3)$ & $42(4)$ & $-17(4)$ \\
\hline$C(7)$ & $23(4)$ & $50(4)$ & $77(5)$ & $-7(3)$ & $15(3)$ & $0(3)$ \\
\hline$C(8)$ & $40(4)$ & 44(4) & 29(3) & $-4(2)$ & $-2(3)$ & $-10(3)$ \\
\hline $\mathrm{C}(9)$ & $45(4)$ & 29(3) & $52(4)$ & $-8(3)$ & $3(3)$ & $-2(3)$ \\
\hline$C(10)$ & $60(4)$ & $50(4)$ & $22(3)$ & $7(2)$ & 1(3) & $-15(3)$ \\
\hline $\mathrm{C}(11)$ & 19(3) & $37(3)$ & $37(3)$ & $10(2)$ & $12(2)$ & 4(3) \\
\hline$C(12)$ & $23(3)$ & 44(3) & $24(2)$ & $-3(2)$ & $6(2)$ & $12(3)$ \\
\hline$C(13)$ & $20(3)$ & 31(3) & $32(3)$ & $3(2)$ & $6(2)$ & $7(2)$ \\
\hline$C(14)$ & $17(3)$ & $40(3)$ & $27(3)$ & $-5(2)$ & $6(2)$ & $1(2)$ \\
\hline$C(15)$ & $16(3)$ & $33(3)$ & 43(3) & $0(2)$ & $14(2)$ & $-1(2)$ \\
\hline$C(16)$ & $35(4)$ & $70(5)$ & $56(4)$ & $31(3)$ & $21(3)$ & $9(3)$ \\
\hline$C(17)$ & $44(4)$ & $68(5)$ & $30(3)$ & $-11(3)$ & 4(3) & $15(3)$ \\
\hline$C(18)$ & $36(4)$ & $37(4)$ & 46(4) & $8(3)$ & $5(3)$ & $9(3)$ \\
\hline$C(19)$ & $35(4)$ & $56(4)$ & $32(3)$ & $-4(2)$ & 1(3) & $-1(3)$ \\
\hline $\mathrm{C}(20)$ & $30(4)$ & $43(4)$ & $80(5)$ & $-12(3)$ & $19(3)$ & $-9(3)$ \\
\hline $\mathrm{C}(21)$ & $33(3)$ & $40(3)$ & $24(3)$ & $-6(2)$ & $9(2)$ & $-7(3)$ \\
\hline $\mathrm{C}(22)$ & $40(4)$ & $34(3)$ & $21(3)$ & $-13(2)$ & $10(2)$ & $-6(3)$ \\
\hline$C(23)$ & $33(4)$ & $39(4)$ & $40(3)$ & $-6(3)$ & $12(3)$ & $-7(3)$ \\
\hline$C(24)$ & $49(4)$ & $40(4)$ & $48(4)$ & $0(3)$ & 21(3) & $-4(3)$ \\
\hline$C(25)$ & $47(4)$ & $45(4)$ & $45(4)$ & $0(3)$ & $13(3)$ & $8(3)$ \\
\hline$C(26)$ & $36(4)$ & $52(4)$ & $46(4)$ & $-8(3)$ & $12(3)$ & $3(3)$ \\
\hline$C(27)$ & $39(4)$ & $42(4)$ & $34(3)$ & $-7(2)$ & $14(3)$ & $-5(3)$ \\
\hline$C(28)$ & $28(3)$ & $38(3)$ & $23(3)$ & $-3(2)$ & $4(2)$ & $4(2)$ \\
\hline$C(29)$ & $28(3)$ & $25(3)$ & $27(3)$ & $6(2)$ & $3(2)$ & $0(2)$ \\
\hline $\mathrm{C}(30)$ & $33(3)$ & $43(4)$ & $34(3)$ & 1(2) & $5(3)$ & $5(3)$ \\
\hline
\end{tabular}




\begin{tabular}{lllllll}
$\mathrm{C}(31)$ & $31(4)$ & $57(4)$ & $45(4)$ & $6(3)$ & $-5(3)$ & $-1(3)$ \\
$\mathrm{C}(32)$ & $53(5)$ & $50(4)$ & $30(3)$ & $-2(3)$ & $-7(3)$ & $-12(3)$ \\
$\mathrm{C}(33)$ & $61(5)$ & $39(4)$ & $32(3)$ & $-5(2)$ & $9(3)$ & $2(3)$ \\
$\mathrm{C}(34)$ & $37(4)$ & $36(4)$ & $30(3)$ & $1(2)$ & $3(2)$ & $5(3)$ \\
\hline
\end{tabular}


Table S25. Hydrogen coordinates ( x 104) and isotropic displacement parameters $\left(\AA^{2} \times 10^{3}\right)$ for $\left(\mathrm{C}_{5} \mathrm{Me}_{5}\right)_{2} \mathrm{U}\left(\mathrm{CH}_{2} \mathrm{Ph}\right)_{2}$ (11b).

\begin{tabular}{|c|c|c|c|c|}
\hline & $\mathrm{x}$ & $\mathrm{y}$ & z & $\mathrm{U}(\mathrm{eq})$ \\
\hline $\mathrm{H}(6 \mathrm{~A})$ & 4940 & 2893 & -262 & 80 \\
\hline $\mathrm{H}(6 \mathrm{~B})$ & 5649 & 1918 & 99 & 80 \\
\hline $\mathrm{H}(6 \mathrm{C})$ & 6492 & 2985 & 49 & 80 \\
\hline $\mathrm{H}(7 \mathrm{~A})$ & 7314 & 2462 & 1779 & 74 \\
\hline $\mathrm{H}(7 \mathrm{~B})$ & 7835 & 2635 & 1206 & 74 \\
\hline $\mathrm{H}(7 \mathrm{C})$ & 6900 & 1632 & 1282 & 74 \\
\hline $\mathrm{H}(8 \mathrm{~A})$ & 6393 & 3538 & 2254 & 58 \\
\hline $\mathrm{H}(8 \mathrm{~B})$ & 4931 & 4023 & 2295 & 58 \\
\hline $\mathrm{H}(8 \mathrm{C})$ & 6085 & 4769 & 2123 & 58 \\
\hline $\mathrm{H}(9 \mathrm{~A})$ & 2850 & 5677 & 976 & 64 \\
\hline $\mathrm{H}(9 \mathrm{~B})$ & 4240 & 5937 & 1393 & 64 \\
\hline $\mathrm{H}(9 \mathrm{C})$ & 3068 & 5269 & 1603 & 64 \\
\hline $\mathrm{H}(10 \mathrm{~A})$ & 2571 & 4929 & 119 & 67 \\
\hline $\mathrm{H}(10 \mathrm{~B})$ & 2646 & 3794 & -168 & 67 \\
\hline $\mathrm{H}(10 \mathrm{C})$ & 3766 & 4681 & -218 & 67 \\
\hline $\mathrm{H}(16 \mathrm{~A})$ & 885 & 1661 & 2433 & 78 \\
\hline $\mathrm{H}(16 \mathrm{~B})$ & 1138 & 623 & 2089 & 78 \\
\hline $\mathrm{H}(16 \mathrm{C})$ & -382 & 1039 & 2080 & 78 \\
\hline $\mathrm{H}(17 \mathrm{~A})$ & 1276 & 3344 & 2444 & 72 \\
\hline $\mathrm{H}(17 \mathrm{~B})$ & 317 & 4237 & 2111 & 72 \\
\hline $\mathrm{H}(17 \mathrm{C})$ & 1917 & 4235 & 2103 & 72 \\
\hline $\mathrm{H}(18 \mathrm{~A})$ & 601 & 5090 & 1160 & 60 \\
\hline $\mathrm{H}(18 \mathrm{~B})$ & -686 & 4710 & 714 & 60 \\
\hline $\mathrm{H}(18 \mathrm{C})$ & 784 & 4792 & 544 & 60 \\
\hline $\mathrm{H}(19 \mathrm{~A})$ & -388 & 1859 & -104 & 62 \\
\hline $\mathrm{H}(19 \mathrm{~B})$ & 444 & 2939 & -134 & 62 \\
\hline $\mathrm{H}(19 \mathrm{C})$ & -1102 & 2980 & -40 & 62 \\
\hline $\mathrm{H}(20 \mathrm{~A})$ & -497 & 508 & 409 & 75 \\
\hline $\mathrm{H}(20 \mathrm{~B})$ & -1182 & 416 & 948 & 75 \\
\hline $\mathrm{H}(20 \mathrm{C})$ & 321 & -40 & 959 & 75 \\
\hline
\end{tabular}




\begin{tabular}{lrrrr}
$\mathrm{H}(21 \mathrm{~A})$ & 1971 & 862 & 159 & 38 \\
$\mathrm{H}(21 \mathrm{~B})$ & 3397 & 1334 & 29 & 38 \\
$\mathrm{H}(23)$ & 2046 & -588 & 879 & 44 \\
$\mathrm{H}(24)$ & 3263 & -1851 & 1479 & 53 \\
$\mathrm{H}(25)$ & 5646 & -1851 & 1652 & 54 \\
$\mathrm{H}(26)$ & -640 & 1180 & 53 \\
$\mathrm{H}(27)$ & 6807 & 613 & 572 & 44 \\
$\mathrm{H}(28 \mathrm{~A})$ & 5630 & 852 & 2077 & 36 \\
$\mathrm{H}(28 \mathrm{~B})$ & 3326 & 785 & 1866 & 36 \\
$\mathrm{H}(30)$ & 4723 & 905 & 2524 & 44 \\
$\mathrm{H}(31)$ & 6708 & 1423 & 3399 & 55 \\
$\mathrm{H}(32)$ & 7878 & 2425 & 3997 & 56 \\
$\mathrm{H}(33)$ & 6796 & 2879 & 3718 & 53 \\
$\mathrm{H}(34)$ & 4485 & 2381 & 2849 & 42 \\
& 3289 & & & \\
\hline
\end{tabular}


Table S26. Crystal data and structure refinement for $\left(\mathrm{C}_{5} \mathrm{Me}_{5}\right)_{2} \mathrm{U}\left(\mathrm{CH}_{3}\right)_{2}(\mathbf{1 1 c})$.

Identification code

Empirical formula

Formula weight

Temperature

Wavelength

Crystal system

Space group

Unit cell dimensions

Volume

$\mathrm{Z}$

Density (calculated)

Absorption coefficient

$\mathrm{F}(000)$

Crystal size

Theta range for data collection

Index ranges

Reflections collected

Independent reflections

Max. and min. transmission

Refinement method

Data / restraints / parameters

Goodness-of-fit on $\mathrm{F}^{2}$

Final $\mathrm{R}$ indices [I $>2 \operatorname{sigma}(\mathrm{I})]$

$\mathrm{R}$ indices (all data)

Largest diff. peak and hole $\operatorname{ccd} 825$

C22 H36 U

538.54

203(2) K

$0.71073 \AA$

tetragonal

I $4 / \mathrm{a}$

$\mathrm{a}=31.797(5) \AA$

$\mathrm{c}=8.438(3) \AA$

8532(3) $\AA^{3}$

16

$1.677 \mathrm{Mg} / \mathrm{m}^{3}$

$7.609 \mathrm{~mm}^{-1}$

4160

$0.24 \times 0.16 \times 0.16 \mathrm{~mm}^{3}$

1.28 to $28.45^{\circ}$.

$-38<=\mathrm{h}<=40,-31<=\mathrm{k}<=41,-10<=\mathrm{l}<=10$

29886

$5026[\mathrm{R}(\mathrm{int})=0.0320]$

0.3756 and 0.2625

Full-matrix least-squares on $\mathrm{F}^{2}$

$5026 / 0 / 208$

1.953

$\mathrm{R} 1=0.0456, \mathrm{wR} 2=0.0922$

$\mathrm{R} 1=0.0572, \mathrm{wR} 2=0.0935$

1.861 and -1.315 e. $\AA^{-3}$ 
Table S27. Atomic coordinates ( x 104) and equivalent isotropic displacement parameters $\left(\AA^{2} \times 10^{3}\right)$ for $\left(\mathrm{C}_{5} \mathrm{Me}_{5}\right)_{2} \mathrm{U}\left(\mathrm{CH}_{3}\right)_{2}$ (11c). U(eq) is defined as one third of the trace of the orthogonalized $\mathrm{U}^{\mathrm{ij}}$ tensor.

\begin{tabular}{|c|c|c|c|c|}
\hline & $\mathrm{x}$ & $\mathrm{y}$ & z & $\mathrm{U}(\mathrm{eq})$ \\
\hline $\mathrm{U}(1)$ & $1490(1)$ & 6494(1) & 1058(1) & $29(1)$ \\
\hline$C(1)$ & 2243(2) & $6688(2)$ & $-260(8)$ & $41(2)$ \\
\hline$C(2)$ & 2349(2) & $6557(2)$ & $1285(7)$ & $36(1)$ \\
\hline$C(3)$ & 2272(2) & $6117(2)$ & $1400(7)$ & $39(2)$ \\
\hline$C(4)$ & 2115(2) & $5985(2)$ & $-74(8)$ & $43(2)$ \\
\hline$C(5)$ & 2099(2) & $6330(3)$ & $-1082(7)$ & $46(2)$ \\
\hline$C(6)$ & 2262(3) & 7134(3) & $-879(11)$ & $69(3)$ \\
\hline$C(7)$ & $2566(2)$ & $6817(3)$ & $2539(10)$ & $56(2)$ \\
\hline$C(8)$ & $2400(3)$ & $5836(3)$ & 2759(9) & $60(2)$ \\
\hline$C(9)$ & 1993(3) & $5530(3)$ & $-469(11)$ & $70(3)$ \\
\hline$C(10)$ & 2014(3) & 6308(3) & $-2873(8)$ & $70(3)$ \\
\hline$C(11)$ & $800(2)$ & $6946(2)$ & $85(8)$ & $34(1)$ \\
\hline$C(12)$ & $931(2)$ & $6715(2)$ & $-1253(7)$ & $36(2)$ \\
\hline$C(13)$ & $855(2)$ & $6284(2)$ & $-979(7)$ & $35(1)$ \\
\hline$C(14)$ & $680(2)$ & $6243(2)$ & $546(7)$ & $32(1)$ \\
\hline$C(15)$ & $642(2)$ & $6655(2)$ & $1208(7)$ & $32(1)$ \\
\hline$C(16)$ & 781(3) & $7415(2)$ & $186(10)$ & $58(2)$ \\
\hline$C(17)$ & 1068(3) & $6910(3)$ & $-2813(9)$ & $63(2)$ \\
\hline$C(18)$ & $902(2)$ & $5927(3)$ & $-2148(9)$ & $55(2)$ \\
\hline$C(19)$ & $509(2)$ & $5844(2)$ & 1261(9) & $53(2)$ \\
\hline$C(20)$ & $443(2)$ & $6756(3)$ & 2778(9) & $54(2)$ \\
\hline$C(21)$ & $1560(2)$ & $7113(3)$ & $2713(10)$ & $57(2)$ \\
\hline$C(22)$ & 1378(2) & $6020(3)$ & $3252(8)$ & $49(2)$ \\
\hline
\end{tabular}


Table S28. Bond lengths $[\AA]$ and angles $\left[^{\circ}\right]$ for $\left(\mathrm{C}_{5} \mathrm{Me}_{5}\right)_{2} \mathrm{U}\left(\mathrm{CH}_{3}\right)_{2}(\mathbf{1 1 c})$.

\begin{tabular}{ll}
\hline $\mathrm{U}(1)-\mathrm{C}(22)$ & $2.414(7)$ \\
$\mathrm{U}(1)-\mathrm{C}(21)$ & $2.424(7)$ \\
$\mathrm{U}(1)-\mathrm{C}(5)$ & $2.696(6)$ \\
$\mathrm{U}(1)-\mathrm{C}(1)$ & $2.710(7)$ \\
$\mathrm{U}(1)-\mathrm{C}(14)$ & $2.732(6)$ \\
$\mathrm{U}(1)-\mathrm{C}(12)$ & $2.731(6)$ \\
$\mathrm{U}(1)-\mathrm{C}(4)$ & $2.736(7)$ \\
$\mathrm{U}(1)-\mathrm{C}(13)$ & $2.735(6)$ \\
$\mathrm{U}(1)-\mathrm{C}(2)$ & $2.745(6)$ \\
$\mathrm{U}(1)-\mathrm{C}(11)$ & $2.748(6)$ \\
$\mathrm{U}(1)-\mathrm{C}(15)$ & $2.749(6)$ \\
$\mathrm{U}(1)-\mathrm{C}(3)$ & $2.774(7)$ \\
$\mathrm{C}(1)-\mathrm{C}(2)$ & $1.411(9)$ \\
$\mathrm{C}(1)-\mathrm{C}(5)$ & $1.410(10)$ \\
$\mathrm{C}(1)-\mathrm{C}(6)$ & $1.513(10)$ \\
$\mathrm{C}(2)-\mathrm{C}(3)$ & $1.424(10)$ \\
$\mathrm{C}(2)-\mathrm{C}(7)$ & $1.510(9)$ \\
$\mathrm{C}(3)-\mathrm{C}(4)$ & $1.404(9)$ \\
$\mathrm{C}(3)-\mathrm{C}(8)$ & $1.509(9)$ \\
$\mathrm{C}(4)-\mathrm{C}(5)$ & $1.390(10)$ \\
$\mathrm{C}(4)-\mathrm{C}(9)$ & $1.532(11)$ \\
$\mathrm{C}(5)-\mathrm{C}(10)$ & $1.537(9)$ \\
$\mathrm{C}(11)-\mathrm{C}(12)$ & $1.409(9)$ \\
$\mathrm{C}(11)-\mathrm{C}(15)$ & $1.417(9)$ \\
$\mathrm{C}(11)-\mathrm{C}(16)$ & $1.496(9)$ \\
$\mathrm{C}(12)-\mathrm{C}(13)$ & $1.408(9)$ \\
$\mathrm{C}(12)-\mathrm{C}(17)$ & $1.521(9)$ \\
$\mathrm{C}(13)-\mathrm{C}(14)$ & $1.408(9)$ \\
$\mathrm{C}(13)-\mathrm{C}(18)$ & \\
$\mathrm{C}(14)-\mathrm{C}(15)$ & \\
$\mathrm{C}(14)-\mathrm{C}(19)$ & \\
$\mathrm{C}(15)-\mathrm{C}(20)$ & \\
& \\
$\mathrm{C}(1)-\mathrm{C}(21)$ & \\
& \\
& \\
&
\end{tabular}




\begin{tabular}{|c|c|}
\hline $\mathrm{C}(22)-\mathrm{U}(1)-\mathrm{C}(5)$ & $119.9(3)$ \\
\hline $\mathrm{C}(21)-\mathrm{U}(1)-\mathrm{C}(5)$ & $118.6(3)$ \\
\hline $\mathrm{C}(22)-\mathrm{U}(1)-\mathrm{C}(1)$ & $126.0(2)$ \\
\hline $\mathrm{C}(21)-\mathrm{U}(1)-\mathrm{C}(1)$ & $88.4(3)$ \\
\hline C(5)-U(1)-C(1) & $30.2(2)$ \\
\hline $\mathrm{C}(22)-\mathrm{U}(1)-\mathrm{C}(14)$ & $78.4(2)$ \\
\hline $\mathrm{C}(21)-\mathrm{U}(1)-\mathrm{C}(14)$ & $114.5(2)$ \\
\hline $\mathrm{C}(5)-\mathrm{U}(1)-\mathrm{C}(14)$ & $120.9(2)$ \\
\hline C(1)-U(1)-C(14) & $146.6(2)$ \\
\hline $\mathrm{C}(22)-\mathrm{U}(1)-\mathrm{C}(12)$ & $127.7(2)$ \\
\hline $\mathrm{C}(21)-\mathrm{U}(1)-\mathrm{C}(12)$ & 105.2(3) \\
\hline C(5)-U(1)-C(12) & $92.2(2)$ \\
\hline $\mathrm{C}(1)-\mathrm{U}(1)-\mathrm{C}(12)$ & $102.95(19)$ \\
\hline $\mathrm{C}(14)-\mathrm{U}(1)-\mathrm{C}(12)$ & $49.27(18)$ \\
\hline $\mathrm{C}(22)-\mathrm{U}(1)-\mathrm{C}(4)$ & $90.3(2)$ \\
\hline $\mathrm{C}(21)-\mathrm{U}(1)-\mathrm{C}(4)$ & $128.1(2)$ \\
\hline $\mathrm{C}(5)-\mathrm{U}(1)-\mathrm{C}(4)$ & $29.7(2)$ \\
\hline $\mathrm{C}(1)-\mathrm{U}(1)-\mathrm{C}(4)$ & $49.5(2)$ \\
\hline $\mathrm{C}(14)-\mathrm{U}(1)-\mathrm{C}(4)$ & $117.2(2)$ \\
\hline $\mathrm{C}(12)-\mathrm{U}(1)-\mathrm{C}(4)$ & $112.1(2)$ \\
\hline $\mathrm{C}(22)-\mathrm{U}(1)-\mathrm{C}(13)$ & $102.7(2)$ \\
\hline $\mathrm{C}(21)-\mathrm{U}(1)-\mathrm{C}(13)$ & $128.9(2)$ \\
\hline C(5)-U(1)-C(13) & $93.5(2)$ \\
\hline $\mathrm{C}(1)-\mathrm{U}(1)-\mathrm{C}(13)$ & $116.7(2)$ \\
\hline $\mathrm{C}(14)-\mathrm{U}(1)-\mathrm{C}(13)$ & $29.85(18)$ \\
\hline $\mathrm{C}(12)-\mathrm{U}(1)-\mathrm{C}(13)$ & $29.8(2)$ \\
\hline C(4)-U(1)-C(13) & $99.9(2)$ \\
\hline $\mathrm{C}(22)-\mathrm{U}(1)-\mathrm{C}(2)$ & $98.0(2)$ \\
\hline $\mathrm{C}(21)-\mathrm{U}(1)-\mathrm{C}(2)$ & $79.1(2)$ \\
\hline $\mathrm{C}(5)-\mathrm{U}(1)-\mathrm{C}(2)$ & $49.3(2)$ \\
\hline $\mathrm{C}(1)-\mathrm{U}(1)-\mathrm{C}(2)$ & 29.96(19) \\
\hline $\mathrm{C}(14)-\mathrm{U}(1)-\mathrm{C}(2)$ & $166.1(2)$ \\
\hline $\mathrm{C}(12)-\mathrm{U}(1)-\mathrm{C}(2)$ & $132.82(19)$ \\
\hline $\mathrm{C}(4)-\mathrm{U}(1)-\mathrm{C}(2)$ & $49.1(2)$ \\
\hline $\mathrm{C}(13)-\mathrm{U}(1)-\mathrm{C}(2)$ & $142.80(19)$ \\
\hline $\mathrm{C}(22)-\mathrm{U}(1)-\mathrm{C}(11)$ & $115.9(2)$ \\
\hline
\end{tabular}




\begin{tabular}{|c|c|}
\hline $\mathrm{C}(21)-\mathrm{U}(1)-\mathrm{C}(11)$ & $79.7(2)$ \\
\hline $\mathrm{C}(5)-\mathrm{U}(1)-\mathrm{C}(11)$ & $118.3(2)$ \\
\hline $\mathrm{C}(1)-\mathrm{U}(1)-\mathrm{C}(11)$ & $117.6(2)$ \\
\hline $\mathrm{C}(14)-\mathrm{U}(1)-\mathrm{C}(11)$ & 49.61(19) \\
\hline $\mathrm{C}(12)-\mathrm{U}(1)-\mathrm{C}(11)$ & 29.81(19) \\
\hline $\mathrm{C}(4)-\mathrm{U}(1)-\mathrm{C}(11)$ & $141.7(2)$ \\
\hline $\mathrm{C}(13)-\mathrm{U}(1)-\mathrm{C}(11)$ & 49.43(19) \\
\hline $\mathrm{C}(2)-\mathrm{U}(1)-\mathrm{C}(11)$ & 141.1(2) \\
\hline$C(22)-U(1)-C(15)$ & $86.3(2)$ \\
\hline $\mathrm{C}(21)-\mathrm{U}(1)-\mathrm{C}(15)$ & $85.0(2)$ \\
\hline $\mathrm{C}(5)-\mathrm{U}(1)-\mathrm{C}(15)$ & $140.41(19)$ \\
\hline $\mathrm{C}(1)-\mathrm{U}(1)-\mathrm{C}(15)$ & $147.5(2)$ \\
\hline $\mathrm{C}(14)-\mathrm{U}(1)-\mathrm{C}(15)$ & $30.18(18)$ \\
\hline $\mathrm{C}(12)-\mathrm{U}(1)-\mathrm{C}(15)$ & $49.14(18)$ \\
\hline $\mathrm{C}(4)-\mathrm{U}(1)-\mathrm{C}(15)$ & $146.98(19)$ \\
\hline $\mathrm{C}(13)-\mathrm{U}(1)-\mathrm{C}(15)$ & $49.39(18)$ \\
\hline $\mathrm{C}(2)-\mathrm{U}(1)-\mathrm{C}(15)$ & $163.73(19)$ \\
\hline $\mathrm{C}(11)-\mathrm{U}(1)-\mathrm{C}(15)$ & $29.89(18)$ \\
\hline $\mathrm{C}(22)-\mathrm{U}(1)-\mathrm{C}(3)$ & $77.4(2)$ \\
\hline $\mathrm{C}(21)-\mathrm{U}(1)-\mathrm{C}(3)$ & $102.1(2)$ \\
\hline $\mathrm{C}(5)-\mathrm{U}(1)-\mathrm{C}(3)$ & $49.0(2)$ \\
\hline $\mathrm{C}(1)-\mathrm{U}(1)-\mathrm{C}(3)$ & $49.5(2)$ \\
\hline C(14)-U(1)-C(3) & $137.3(2)$ \\
\hline $\mathrm{C}(12)-\mathrm{U}(1)-\mathrm{C}(3)$ & $140.23(19)$ \\
\hline $\mathrm{C}(4)-\mathrm{U}(1)-\mathrm{C}(3)$ & 29.51(19) \\
\hline $\mathrm{C}(13)-\mathrm{U}(1)-\mathrm{C}(3)$ & $128.4(2)$ \\
\hline $\mathrm{C}(2)-\mathrm{U}(1)-\mathrm{C}(3)$ & $29.9(2)$ \\
\hline $\mathrm{C}(11)-\mathrm{U}(1)-\mathrm{C}(3)$ & $166.49(19)$ \\
\hline $\mathrm{C}(15)-\mathrm{U}(1)-\mathrm{C}(3)$ & $162.6(2)$ \\
\hline$C(2)-C(1)-C(5)$ & 107.1(6) \\
\hline $\mathrm{C}(2)-\mathrm{C}(1)-\mathrm{C}(6)$ & $125.9(7)$ \\
\hline$C(5)-C(1)-C(6)$ & $126.9(7)$ \\
\hline $\mathrm{C}(2)-\mathrm{C}(1)-\mathrm{U}(1)$ & 76.4(4) \\
\hline $\mathrm{C}(5)-\mathrm{C}(1)-\mathrm{U}(1)$ & 74.4(4) \\
\hline $\mathrm{C}(6)-\mathrm{C}(1)-\mathrm{U}(1)$ & $113.0(5)$ \\
\hline $\mathrm{C}(1)-\mathrm{C}(2)-\mathrm{C}(3)$ & $108.2(6)$ \\
\hline
\end{tabular}




\begin{tabular}{|c|c|}
\hline $\mathrm{C}(1)-\mathrm{C}(2)-\mathrm{C}(7)$ & $126.5(7)$ \\
\hline$C(3)-C(2)-C(7)$ & $124.8(6)$ \\
\hline $\mathrm{C}(1)-\mathrm{C}(2)-\mathrm{U}(1)$ & $73.6(4)$ \\
\hline $\mathrm{C}(3)-\mathrm{C}(2)-\mathrm{U}(1)$ & $76.2(4)$ \\
\hline $\mathrm{C}(7)-\mathrm{C}(2)-\mathrm{U}(1)$ & $122.9(4)$ \\
\hline $\mathrm{C}(4)-\mathrm{C}(3)-\mathrm{C}(2)$ & $107.2(6)$ \\
\hline $\mathrm{C}(4)-\mathrm{C}(3)-\mathrm{C}(8)$ & $126.3(7)$ \\
\hline $\mathrm{C}(2)-\mathrm{C}(3)-\mathrm{C}(8)$ & $125.8(7)$ \\
\hline $\mathrm{C}(4)-\mathrm{C}(3)-\mathrm{U}(1)$ & $73.7(4)$ \\
\hline $\mathrm{C}(2)-\mathrm{C}(3)-\mathrm{U}(1)$ & $73.9(4)$ \\
\hline $\mathrm{C}(8)-\mathrm{C}(3)-\mathrm{U}(1)$ & $125.3(5)$ \\
\hline$C(5)-C(4)-C(3)$ & $108.6(7)$ \\
\hline C(5)-C(4)-C(9) & $127.0(7)$ \\
\hline $\mathrm{C}(3)-\mathrm{C}(4)-\mathrm{C}(9)$ & $124.4(7)$ \\
\hline $\mathrm{C}(5)-\mathrm{C}(4)-\mathrm{U}(1)$ & $73.6(4)$ \\
\hline $\mathrm{C}(3)-\mathrm{C}(4)-\mathrm{U}(1)$ & $76.8(4)$ \\
\hline $\mathrm{C}(9)-\mathrm{C}(4)-\mathrm{U}(1)$ & $116.8(5)$ \\
\hline$C(4)-C(5)-C(1)$ & $109.0(6)$ \\
\hline $\mathrm{C}(4)-\mathrm{C}(5)-\mathrm{C}(10)$ & $125.0(8)$ \\
\hline $\mathrm{C}(1)-\mathrm{C}(5)-\mathrm{C}(10)$ & $125.2(8)$ \\
\hline $\mathrm{C}(4)-\mathrm{C}(5)-\mathrm{U}(1)$ & $76.7(4)$ \\
\hline $\mathrm{C}(1)-\mathrm{C}(5)-\mathrm{U}(1)$ & $75.4(4)$ \\
\hline $\mathrm{C}(10)-\mathrm{C}(5)-\mathrm{U}(1)$ & $122.9(5)$ \\
\hline$C(12)-C(11)-C(15)$ & $107.4(6)$ \\
\hline$C(12)-C(11)-C(16)$ & $125.4(6)$ \\
\hline$C(15)-C(11)-C(16)$ & $126.7(6)$ \\
\hline $\mathrm{C}(12)-\mathrm{C}(11)-\mathrm{U}(1)$ & $74.4(4)$ \\
\hline $\mathrm{C}(15)-\mathrm{C}(11)-\mathrm{U}(1)$ & $75.1(4)$ \\
\hline $\mathrm{C}(16)-\mathrm{C}(11)-\mathrm{U}(1)$ & $122.5(5)$ \\
\hline$C(13)-C(12)-C(11)$ & $109.0(5)$ \\
\hline $\mathrm{C}(13)-\mathrm{C}(12)-\mathrm{C}(17)$ & $126.1(7)$ \\
\hline$C(11)-C(12)-C(17)$ & $124.4(7)$ \\
\hline $\mathrm{C}(13)-\mathrm{C}(12)-\mathrm{U}(1)$ & $75.2(4)$ \\
\hline $\mathrm{C}(11)-\mathrm{C}(12)-\mathrm{U}(1)$ & $75.8(4)$ \\
\hline$C(17)-C(12)-U(1)$ & $122.4(5)$ \\
\hline$C(12)-C(13)-C(14)$ & $107.9(5)$ \\
\hline
\end{tabular}




$\begin{array}{lr}\mathrm{C}(12)-\mathrm{C}(13)-\mathrm{C}(18) & 127.3(6) \\ \mathrm{C}(14)-\mathrm{C}(13)-\mathrm{C}(18) & 124.4(6) \\ \mathrm{C}(12)-\mathrm{C}(13)-\mathrm{U}(1) & 74.9(3) \\ \mathrm{C}(14)-\mathrm{C}(13)-\mathrm{U}(1) & 75.0(3) \\ \mathrm{C}(18)-\mathrm{C}(13)-\mathrm{U}(1) & 121.4(4) \\ \mathrm{C}(13)-\mathrm{C}(14)-\mathrm{C}(15) & 107.8(5) \\ \mathrm{C}(13)-\mathrm{C}(14)-\mathrm{C}(19) & 125.9(6) \\ \mathrm{C}(15)-\mathrm{C}(14)-\mathrm{C}(19) & 125.8(6) \\ \mathrm{C}(13)-\mathrm{C}(14)-\mathrm{U}(1) & 75.2(3) \\ \mathrm{C}(15)-\mathrm{C}(14)-\mathrm{U}(1) & 75.5(3) \\ \mathrm{C}(19)-\mathrm{C}(14)-\mathrm{U}(1) & 121.5(4) \\ \mathrm{C}(11)-\mathrm{C}(15)-\mathrm{C}(14) & 107.9(5) \\ \mathrm{C}(11)-\mathrm{C}(15)-\mathrm{C}(20) & 126.8(6) \\ \mathrm{C}(14)-\mathrm{C}(15)-\mathrm{C}(20) & 125.3(6) \\ \mathrm{C}(11)-\mathrm{C}(15)-\mathrm{U}(1) & 75.0(4) \\ \mathrm{C}(14)-\mathrm{C}(15)-\mathrm{U}(1) & 74.3(3) \\ \mathrm{C}(20)-\mathrm{C}(15)-\mathrm{U}(1) & 119.6(4) \\ & \end{array}$

Symmetry transformations used to generate equivalent atoms: 
Table S29. Anisotropic displacement parameters $\left(\AA^{2} \times 10^{3}\right)$ for $\left(\mathrm{C}_{5} \mathrm{Me}_{5}\right)_{2} \mathrm{U}_{(}\left(\mathrm{CH}_{3}\right)_{2}$ (11c). The anisotropic displacement factor exponent takes the form: $-2 \pi^{2}\left[h^{2} a^{* 2} U^{11}+\ldots+2 h k a^{*} b^{*} U^{12}\right]$

\begin{tabular}{|c|c|c|c|c|c|c|}
\hline & $\mathrm{U}^{11}$ & $\mathrm{U}^{22}$ & $\mathrm{U}^{33}$ & $\mathrm{U}^{23}$ & $\mathrm{U}^{13}$ & $\mathrm{U}^{12}$ \\
\hline $\mathrm{U}(1)$ & $26(1)$ & $34(1)$ & $26(1)$ & $-3(1)$ & 1(1) & $-4(1)$ \\
\hline$C(1)$ & $26(3)$ & $53(5)$ & 44(4) & $12(3)$ & $4(3)$ & $-2(3)$ \\
\hline$C(2)$ & $23(3)$ & $44(4)$ & $42(3)$ & $-2(3)$ & $-1(3)$ & $2(3)$ \\
\hline$C(3)$ & $35(4)$ & $42(4)$ & $40(3)$ & $2(3)$ & $7(3)$ & 2(3) \\
\hline$C(4)$ & $33(4)$ & $56(5)$ & $40(4)$ & $-13(3)$ & $6(3)$ & $2(3)$ \\
\hline$C(5)$ & $28(3)$ & $82(6)$ & $28(3)$ & $-1(3)$ & $7(3)$ & $8(4)$ \\
\hline$C(6)$ & $43(5)$ & $76(6)$ & $87(6)$ & $46(5)$ & $14(4)$ & $5(4)$ \\
\hline$C(7)$ & $32(4)$ & $68(6)$ & $69(5)$ & $-15(4)$ & $-15(4)$ & $-3(4)$ \\
\hline$C(8)$ & $55(5)$ & $67(6)$ & $58(5)$ & $19(4)$ & $4(4)$ & $17(4)$ \\
\hline C(9) & $58(6)$ & $64(6)$ & $87(6)$ & $-29(5)$ & $4(5)$ & $5(5)$ \\
\hline$C(10)$ & $40(5)$ & $138(9)$ & $32(4)$ & $-2(5)$ & $3(3)$ & $13(5)$ \\
\hline $\mathrm{C}(11)$ & $27(3)$ & $27(3)$ & $47(4)$ & $0(3)$ & $-2(3)$ & $4(3)$ \\
\hline$C(12)$ & $32(3)$ & $45(4)$ & $31(3)$ & $8(3)$ & $-3(3)$ & $-4(3)$ \\
\hline$C(13)$ & $31(3)$ & $39(4)$ & $35(3)$ & $-10(3)$ & $-9(3)$ & $1(3)$ \\
\hline$C(14)$ & $27(3)$ & $26(3)$ & $42(3)$ & $3(3)$ & $-3(3)$ & $-11(3)$ \\
\hline$C(15)$ & $24(3)$ & $32(3)$ & $39(3)$ & $-5(3)$ & $-2(3)$ & $0(3)$ \\
\hline$C(16)$ & $60(5)$ & $37(4)$ & $76(6)$ & $5(4)$ & $-6(4)$ & $4(4)$ \\
\hline$C(17)$ & $49(5)$ & $92(7)$ & $46(4)$ & $36(4)$ & $-1(4)$ & $0(5)$ \\
\hline$C(18)$ & $46(5)$ & $61(5)$ & $57(4)$ & $-34(4)$ & $-3(4)$ & $-2(4)$ \\
\hline$C(19)$ & $48(5)$ & $46(4)$ & $67(5)$ & $19(4)$ & $-11(4)$ & $-13(4)$ \\
\hline$C(20)$ & $31(4)$ & $75(6)$ & $55(4)$ & $-20(4)$ & $12(3)$ & $-2(4)$ \\
\hline$C(21)$ & $38(4)$ & $66(6)$ & $67(5)$ & $-32(4)$ & $1(4)$ & $2(4)$ \\
\hline$C(22)$ & $37(4)$ & $73(6)$ & $37(4)$ & $15(4)$ & $2(3)$ & $-7(4)$ \\
\hline
\end{tabular}


Table S30. Hydrogen coordinates ( x 104) and isotropic displacement parameters $\left(\AA^{2} \times 10^{3}\right)$ for $\left(\mathrm{C}_{5} \mathrm{Me}_{5}\right)_{2} \mathrm{U}\left(\mathrm{CH}_{3}\right)_{2}$ (11c).

\begin{tabular}{|c|c|c|c|c|}
\hline & $\mathrm{x}$ & $\mathrm{y}$ & z & $\mathrm{U}(\mathrm{eq})$ \\
\hline $\mathrm{H}(6 \mathrm{~A})$ & 2173 & 7139 & -1966 & 103 \\
\hline $\mathrm{H}(6 \mathrm{~B})$ & 2546 & 7237 & -806 & 103 \\
\hline $\mathrm{H}(6 \mathrm{C})$ & 2080 & 7310 & -258 & 103 \\
\hline $\mathrm{H}(7 \mathrm{~A})$ & 2601 & 6652 & 3482 & 84 \\
\hline $\mathrm{H}(7 \mathrm{~B})$ & 2399 & 7060 & 2775 & 84 \\
\hline $\mathrm{H}(7 \mathrm{C})$ & 2837 & 6905 & 2157 & 84 \\
\hline $\mathrm{H}(8 \mathrm{~A})$ & 2502 & 6006 & 3618 & 90 \\
\hline $\mathrm{H}(8 \mathrm{~B})$ & 2619 & 5649 & 2416 & 90 \\
\hline $\mathrm{H}(8 \mathrm{C})$ & 2162 & 5676 & 3107 & 90 \\
\hline $\mathrm{H}(9 \mathrm{~A})$ & 2032 & 5357 & 451 & 105 \\
\hline $\mathrm{H}(9 \mathrm{~B})$ & 2168 & 5429 & -1315 & 105 \\
\hline $\mathrm{H}(9 \mathrm{C})$ & 1704 & 5521 & -791 & 105 \\
\hline $\mathrm{H}(10 \mathrm{~A})$ & 1922 & 6030 & -3150 & 105 \\
\hline $\mathrm{H}(10 \mathrm{~B})$ & 2268 & 6373 & -3439 & 105 \\
\hline $\mathrm{H}(10 \mathrm{C})$ & 1800 & 6508 & -3150 & 105 \\
\hline $\mathrm{H}(16 \mathrm{~A})$ & 691 & 7496 & 1229 & 87 \\
\hline $\mathrm{H}(16 \mathrm{~B})$ & 584 & 7519 & -583 & 87 \\
\hline $\mathrm{H}(16 \mathrm{C})$ & 1054 & 7531 & -21 & 87 \\
\hline $\mathrm{H}(17 \mathrm{~A})$ & 1096 & 7209 & -2685 & 94 \\
\hline $\mathrm{H}(17 \mathrm{~B})$ & 862 & 6852 & -3614 & 94 \\
\hline $\mathrm{H}(17 \mathrm{C})$ & 1334 & 6793 & -3126 & 94 \\
\hline $\mathrm{H}(18 \mathrm{~A})$ & 833 & 5666 & -1637 & 82 \\
\hline $\mathrm{H}(18 \mathrm{~B})$ & 1187 & 5917 & -2521 & 82 \\
\hline $\mathrm{H}(18 \mathrm{C})$ & 716 & 5972 & -3027 & 82 \\
\hline $\mathrm{H}(19 \mathrm{~A})$ & 407 & 5900 & 2311 & 80 \\
\hline $\mathrm{H}(19 \mathrm{~B})$ & 728 & 5636 & 1308 & 80 \\
\hline $\mathrm{H}(19 \mathrm{C})$ & 282 & 5740 & 617 & 80 \\
\hline $\mathrm{H}(20 \mathrm{~A})$ & 361 & 6500 & 3296 & 80 \\
\hline $\mathrm{H}(20 \mathrm{~B})$ & 200 & 6929 & 2611 & 80 \\
\hline $\mathrm{H}(20 \mathrm{C})$ & 641 & 6904 & 3430 & 80 \\
\hline
\end{tabular}




\begin{tabular}{lllll}
$\mathrm{H}(21 \mathrm{~A})$ & 1610 & 7355 & 2058 & 86 \\
$\mathrm{H}(21 \mathrm{~B})$ & 1791 & 7076 & 3430 & 86 \\
$\mathrm{H}(21 \mathrm{C})$ & 1305 & 7154 & 3307 & 86 \\
$\mathrm{H}(22 \mathrm{~A})$ & 1341 & 5739 & 2857 & 73 \\
$\mathrm{H}(22 \mathrm{~B})$ & 1131 & 6104 & 3825 & 73 \\
$\mathrm{H}(22 \mathrm{C})$ & 1617 & 6028 & 3945 & 73 \\
\hline
\end{tabular}


Table S31. Crystal data and structure refinement for $\left(\mathrm{C}_{5} \mathrm{Me}_{5}\right)_{2} \mathrm{U}[-\mathrm{N}=\mathrm{C}(\mathrm{Ph})(\mathrm{Bz})]_{2}$ (12b).

Identification code

Empirical formula

Formula weight

Temperature

Wavelength

Crystal system

Space group

Unit cell dimensions

Volume

$\mathrm{Z}$

Density (calculated)

Absorption coefficient

$\mathrm{F}(000)$

Crystal size

Theta range for data collection

Index ranges

Reflections collected

Independent reflections

Max. and min. transmission

Refinement method

Data / restraints / parameters

Goodness-of-fit on $\mathrm{F}^{2}$

Final $\mathrm{R}$ indices [I $>2 \operatorname{sigma}(\mathrm{I})]$

$\mathrm{R}$ indices (all data)

Largest diff. peak and hole $\operatorname{ccd} 812$

$\mathrm{C}_{48} \mathrm{H}_{54} \mathrm{~N}_{2} \mathrm{U}$

896.96

203(2) K

$0.71073 \AA$

monoclinic

$\mathrm{C} 2 / \mathrm{c}$

$\mathrm{a}=15.272(3) \AA$

$\alpha=90^{\circ}$.

$\mathrm{b}=13.469(3) \AA$

$\beta=94.784(4)^{\circ}$.

$\mathrm{c}=19.301(4) \AA$

$\gamma=90^{\circ}$.

3956.3(14) $\AA^{3}$

4

$1.506 \mathrm{Mg} / \mathrm{m}^{3}$

$4.138 \mathrm{~mm}^{-1}$

1792

$0.14 \times 0.10 \times 0.04 \mathrm{~mm}^{3}$

2.02 to $25.35^{\circ}$.

$-18<=\mathrm{h}<=18,-16<=\mathrm{k}<=16,-21<=1<=22$

12464

$3507[\mathrm{R}(\mathrm{int})=0.0256]$

0.8520 and 0.5950

Full-matrix least-squares on $\mathrm{F}^{2}$

$3507 / 0 / 231$

1.208

$\mathrm{R} 1=0.0236, \mathrm{wR} 2=0.0550$

$\mathrm{R} 1=0.0275, \mathrm{wR} 2=0.0560$

0.791 and -1.151 e. $\AA^{-3}$ 
Table S32. Atomic coordinates ( x 104) and equivalent isotropic displacement parameters $\left(\AA^{2} \times 10^{3}\right)$ for $\left(\mathrm{C}_{5} \mathrm{Me}_{5}\right)_{2} \mathrm{U}[-\mathrm{N}=\mathrm{C}(\mathrm{Ph})(\mathrm{Bz})]_{2}(\mathbf{1 2 b}) . \mathrm{U}(\mathrm{eq})$ is defined as one third of the trace of the orthogonalized $\mathrm{U}^{\mathrm{ij}}$ tensor.

\begin{tabular}{|c|c|c|c|c|}
\hline & $\mathrm{x}$ & $\mathrm{y}$ & $\mathrm{z}$ & $\mathrm{U}(\mathrm{eq})$ \\
\hline $\mathrm{N}(1)$ & $-4242(2)$ & $573(2)$ & $3198(2)$ & $36(1)$ \\
\hline $\mathrm{U}(1)$ & -5000 & $1589(1)$ & 2500 & $28(1)$ \\
\hline $\mathrm{C}(1)$ & $-4093(2)$ & $1717(2)$ & $1313(2)$ & $36(1)$ \\
\hline$C(2)$ & $-3454(2)$ & $1452(3)$ & $1861(2)$ & $39(1)$ \\
\hline$C(3)$ & $-3341(2)$ & 2261(3) & $2323(2)$ & $41(1)$ \\
\hline$C(4)$ & $-3900(2)$ & $3038(3)$ & $2061(2)$ & $38(1)$ \\
\hline$C(5)$ & $-4378(2)$ & $2690(3)$ & $1446(2)$ & $35(1)$ \\
\hline$C(6)$ & $-4348(3)$ & $1120(3)$ & $668(2)$ & $46(1)$ \\
\hline$C(7)$ & $-2933(3)$ & $507(3)$ & $1898(2)$ & $57(1)$ \\
\hline $\mathrm{C}(8)$ & $-2707(2)$ & $2322(4)$ & $2965(2)$ & $61(1)$ \\
\hline$C(9)$ & $-3877(3)$ & $4090(3)$ & $2320(2)$ & $60(1)$ \\
\hline$C(10)$ & $-5022(3)$ & $3285(3)$ & $985(2)$ & $47(1)$ \\
\hline$C(11)$ & $-3807(2)$ & $-177(3)$ & $3437(2)$ & $35(1)$ \\
\hline $\mathrm{C}(12)$ & $-3167(2)$ & $-127(3)$ & $4069(2)$ & $38(1)$ \\
\hline$C(13)$ & $-2629(2)$ & $-913(3)$ & $4286(2)$ & $50(1)$ \\
\hline$C(14)$ & $-2020(3)$ & $-823(4)$ & $4859(3)$ & $65(1)$ \\
\hline$C(15)$ & $-1945(3)$ & $61(4)$ & $5223(2)$ & $64(1)$ \\
\hline$C(16)$ & $-2477(3)$ & $842(4)$ & $5017(2)$ & $57(1)$ \\
\hline$C(17)$ & $-3085(3)$ & $757(3)$ & $4456(2)$ & $47(1)$ \\
\hline$C(18)$ & $-3902(3)$ & $-1178(3)$ & $3067(2)$ & $45(1)$ \\
\hline$C(19)$ & $-4433(2)$ & $-1960(3)$ & $3417(2)$ & $39(1)$ \\
\hline$C(20)$ & $-4254(3)$ & $-2950(3)$ & $3319(3)$ & $59(1)$ \\
\hline $\mathrm{C}(21)$ & $-4767(4)$ & $-3677(3)$ & 3591(3) & $68(1)$ \\
\hline $\mathrm{C}(22)$ & $-5459(3)$ & $-3433(3)$ & 3961(3) & $58(1)$ \\
\hline$C(23)$ & $-5638(3)$ & $-2449(4)$ & $4060(2)$ & $58(1)$ \\
\hline $\mathrm{C}(24)$ & $-5134(3)$ & $-1721(3)$ & $3790(2)$ & $48(1)$ \\
\hline
\end{tabular}


Table S33. Bond lengths $[\AA]$ and angles $\left[{ }^{\circ}\right]$ for $\left(\mathrm{C}_{5} \mathrm{Me}_{5}\right)_{2} \mathrm{U}[-\mathrm{N}=\mathrm{C}(\mathrm{Ph})(\mathrm{Bz})]_{2}$ (12b).

\begin{tabular}{|c|c|}
\hline $\mathrm{N}(1)-\mathrm{C}(11)$ & $1.274(4)$ \\
\hline $\mathrm{N}(1)-\mathrm{U}(1)$ & $2.184(3)$ \\
\hline $\mathrm{U}(1)-\mathrm{N}(1) \# 1$ & $2.184(3)$ \\
\hline $\mathrm{U}(1)-\mathrm{C}(3)$ & $2.738(3)$ \\
\hline $\mathrm{U}(1)-\mathrm{C}(3) \# 1$ & $2.738(3)$ \\
\hline $\mathrm{U}(1)-\mathrm{C}(5)$ & $2.749(3)$ \\
\hline $\mathrm{U}(1)-\mathrm{C}(5) \# 1$ & $2.749(3)$ \\
\hline $\mathrm{U}(1)-\mathrm{C}(4)$ & $2.754(3)$ \\
\hline $\mathrm{U}(1)-\mathrm{C}(4) \# 1$ & $2.754(3)$ \\
\hline $\mathrm{U}(1)-\mathrm{C}(2) \# 1$ & $2.760(3)$ \\
\hline $\mathrm{U}(1)-\mathrm{C}(2)$ & $2.760(3)$ \\
\hline $\mathrm{U}(1)-\mathrm{C}(1)$ & $2.779(4)$ \\
\hline $\mathrm{U}(1)-\mathrm{C}(1) \# 1$ & $2.779(4)$ \\
\hline$C(1)-C(5)$ & $1.411(5)$ \\
\hline $\mathrm{C}(1)-\mathrm{C}(2)$ & $1.424(5)$ \\
\hline$C(1)-C(6)$ & $1.506(5)$ \\
\hline$C(2)-C(3)$ & $1.410(6)$ \\
\hline$C(2)-C(7)$ & $1.499(5)$ \\
\hline$C(3)-C(4)$ & $1.417(5)$ \\
\hline$C(3)-C(8)$ & $1.510(5)$ \\
\hline $\mathrm{C}(4)-\mathrm{C}(5)$ & $1.420(5)$ \\
\hline$C(4)-C(9)$ & $1.502(6)$ \\
\hline $\mathrm{C}(5)-\mathrm{C}(10)$ & $1.503(5)$ \\
\hline $\mathrm{C}(11)-\mathrm{C}(12)$ & $1.501(5)$ \\
\hline$C(11)-C(18)$ & $1.526(5)$ \\
\hline$C(12)-C(13)$ & $1.383(5)$ \\
\hline$C(12)-C(17)$ & $1.405(5)$ \\
\hline$C(13)-C(14)$ & $1.390(6)$ \\
\hline$C(14)-C(15)$ & $1.383(7)$ \\
\hline$C(15)-C(16)$ & $1.367(7)$ \\
\hline$C(16)-C(17)$ & $1.372(6)$ \\
\hline$C(18)-C(19)$ & $1.521(5)$ \\
\hline$C(19)-C(24)$ & $1.377(6)$ \\
\hline$C(19)-C(20)$ & $1.377(6)$ \\
\hline
\end{tabular}




\begin{tabular}{|c|c|}
\hline$C(20)-C(21)$ & $1.385(7)$ \\
\hline$C(21)-C(22)$ & $1.363(7)$ \\
\hline$C(22)-C(23)$ & $1.371(6)$ \\
\hline$C(23)-C(24)$ & $1.374(6)$ \\
\hline $\mathrm{C}(11)-\mathrm{N}(1)-\mathrm{U}(1)$ & $162.4(3)$ \\
\hline $\mathrm{N}(1) \# 1-\mathrm{U}(1)-\mathrm{N}(1)$ & $102.40(15)$ \\
\hline $\mathrm{N}(1) \# 1-\mathrm{U}(1)-\mathrm{C}(3)$ & $125.33(12)$ \\
\hline $\mathrm{N}(1)-\mathrm{U}(1)-\mathrm{C}(3)$ & $80.55(11)$ \\
\hline $\mathrm{N}(1) \# 1-\mathrm{U}(1)-\mathrm{C}(3) \# 1$ & $80.55(11)$ \\
\hline $\mathrm{N}(1)-\mathrm{U}(1)-\mathrm{C}(3) \# 1$ & $125.33(12)$ \\
\hline $\mathrm{C}(3)-\mathrm{U}(1)-\mathrm{C}(3) \# 1$ & $141.41(17)$ \\
\hline $\mathrm{N}(1) \# 1-\mathrm{U}(1)-\mathrm{C}(5)$ & $94.56(11)$ \\
\hline $\mathrm{N}(1)-\mathrm{U}(1)-\mathrm{C}(5)$ & $126.60(10)$ \\
\hline $\mathrm{C}(3)-\mathrm{U}(1)-\mathrm{C}(5)$ & $49.38(10)$ \\
\hline $\mathrm{C}(3) \# 1-\mathrm{U}(1)-\mathrm{C}(5)$ & $107.14(11)$ \\
\hline $\mathrm{N}(1) \# 1-\mathrm{U}(1)-\mathrm{C}(5) \# 1$ & $126.60(10)$ \\
\hline $\mathrm{N}(1)-\mathrm{U}(1)-\mathrm{C}(5) \# 1$ & $94.56(11)$ \\
\hline $\mathrm{C}(3)-\mathrm{U}(1)-\mathrm{C}(5) \# 1$ & $107.14(11)$ \\
\hline $\mathrm{C}(3) \# 1-\mathrm{U}(1)-\mathrm{C}(5) \# 1$ & $49.38(10)$ \\
\hline $\mathrm{C}(5)-\mathrm{U}(1)-\mathrm{C}(5) \# 1$ & $114.74(15)$ \\
\hline $\mathrm{N}(1) \# 1-\mathrm{U}(1)-\mathrm{C}(4)$ & $124.04(11)$ \\
\hline $\mathrm{N}(1)-\mathrm{U}(1)-\mathrm{C}(4)$ & $109.17(11)$ \\
\hline$C(3)-U(1)-C(4)$ & $29.90(11)$ \\
\hline $\mathrm{C}(3) \# 1-\mathrm{U}(1)-\mathrm{C}(4)$ & $113.49(12)$ \\
\hline$C(5)-U(1)-C(4)$ & 29.91(11) \\
\hline $\mathrm{C}(5) \# 1-\mathrm{U}(1)-\mathrm{C}(4)$ & $95.89(11)$ \\
\hline $\mathrm{N}(1) \# 1-\mathrm{U}(1)-\mathrm{C}(4) \# 1$ & $109.17(11)$ \\
\hline $\mathrm{N}(1)-\mathrm{U}(1)-\mathrm{C}(4) \# 1$ & $124.04(11)$ \\
\hline $\mathrm{C}(3)-\mathrm{U}(1)-\mathrm{C}(4) \# 1$ & $113.49(12)$ \\
\hline $\mathrm{C}(3) \# 1-\mathrm{U}(1)-\mathrm{C}(4) \# 1$ & $29.90(11)$ \\
\hline $\mathrm{C}(5)-\mathrm{U}(1)-\mathrm{C}(4) \# 1$ & $95.89(11)$ \\
\hline $\mathrm{C}(5) \# 1-\mathrm{U}(1)-\mathrm{C}(4) \# 1$ & $29.91(11)$ \\
\hline $\mathrm{C}(4)-\mathrm{U}(1)-\mathrm{C}(4) \# 1$ & $89.75(16)$ \\
\hline $\mathrm{N}(1) \# 1-\mathrm{U}(1)-\mathrm{C}(2) \# 1$ & $78.73(11)$ \\
\hline $\mathrm{N}(1)-\mathrm{U}(1)-\mathrm{C}(2) \# 1$ & $96.40(11)$ \\
\hline
\end{tabular}




\begin{tabular}{|c|c|}
\hline $\mathrm{C}(3)-\mathrm{U}(1)-\mathrm{C}(2) \# 1$ & $155.91(12)$ \\
\hline $\mathrm{C}(3) \# 1-\mathrm{U}(1)-\mathrm{C}(2) \# 1$ & $29.71(12)$ \\
\hline $\mathrm{C}(5)-\mathrm{U}(1)-\mathrm{C}(2) \# 1$ & $136.73(11)$ \\
\hline $\mathrm{C}(5) \# 1-\mathrm{U}(1)-\mathrm{C}(2) \# 1$ & $49.02(10)$ \\
\hline $\mathrm{C}(4)-\mathrm{U}(1)-\mathrm{C}(2) \# 1$ & $138.71(11)$ \\
\hline $\mathrm{C}(4) \# 1-\mathrm{U}(1)-\mathrm{C}(2) \# 1$ & $48.98(11)$ \\
\hline N(1)\#1-U(1)-C(2) & $96.40(11)$ \\
\hline $\mathrm{N}(1)-\mathrm{U}(1)-\mathrm{C}(2)$ & $78.73(11)$ \\
\hline $\mathrm{C}(3)-\mathrm{U}(1)-\mathrm{C}(2)$ & $29.71(12)$ \\
\hline $\mathrm{C}(3) \# 1-\mathrm{U}(1)-\mathrm{C}(2)$ & $155.91(12)$ \\
\hline $\mathrm{C}(5)-\mathrm{U}(1)-\mathrm{C}(2)$ & $49.02(10)$ \\
\hline $\mathrm{C}(5) \# 1-\mathrm{U}(1)-\mathrm{C}(2)$ & $136.73(11)$ \\
\hline $\mathrm{C}(4)-\mathrm{U}(1)-\mathrm{C}(2)$ & $48.98(11)$ \\
\hline C(4)\#1-U(1)-C(2) & $138.71(11)$ \\
\hline $\mathrm{C}(2) \# 1-\mathrm{U}(1)-\mathrm{C}(2)$ & $172.31(15)$ \\
\hline $\mathrm{N}(1) \# 1-\mathrm{U}(1)-\mathrm{C}(1)$ & $78.74(11)$ \\
\hline N(1)-U(1)-C(1) & $105.81(10)$ \\
\hline $\mathrm{C}(3)-\mathrm{U}(1)-\mathrm{C}(1)$ & $49.23(11)$ \\
\hline $\mathrm{C}(3) \# 1-\mathrm{U}(1)-\mathrm{C}(1)$ & $127.76(11)$ \\
\hline $\mathrm{C}(5)-\mathrm{U}(1)-\mathrm{C}(1)$ & $29.58(10)$ \\
\hline $\mathrm{C}(5) \# 1-\mathrm{U}(1)-\mathrm{C}(1)$ & $143.44(10)$ \\
\hline $\mathrm{C}(4)-\mathrm{U}(1)-\mathrm{C}(1)$ & $49.03(11)$ \\
\hline $\mathrm{C}(4) \# 1-\mathrm{U}(1)-\mathrm{C}(1)$ & $124.63(11)$ \\
\hline $\mathrm{C}(2) \# 1-\mathrm{U}(1)-\mathrm{C}(1)$ & $151.19(11)$ \\
\hline $\mathrm{C}(2)-\mathrm{U}(1)-\mathrm{C}(1)$ & $29.78(11)$ \\
\hline $\mathrm{N}(1) \# 1-\mathrm{U}(1)-\mathrm{C}(1) \# 1$ & $105.81(10)$ \\
\hline $\mathrm{N}(1)-\mathrm{U}(1)-\mathrm{C}(1) \# 1$ & $78.74(11)$ \\
\hline $\mathrm{C}(3)-\mathrm{U}(1)-\mathrm{C}(1) \# 1$ & $127.76(11)$ \\
\hline $\mathrm{C}(3) \# 1-\mathrm{U}(1)-\mathrm{C}(1) \# 1$ & $49.23(11)$ \\
\hline $\mathrm{C}(5)-\mathrm{U}(1)-\mathrm{C}(1) \# 1$ & $143.44(10)$ \\
\hline $\mathrm{C}(5) \# 1-\mathrm{U}(1)-\mathrm{C}(1) \# 1$ & $29.58(10)$ \\
\hline $\mathrm{C}(4)-\mathrm{U}(1)-\mathrm{C}(1) \# 1$ & $124.63(11)$ \\
\hline $\mathrm{C}(4) \# 1-\mathrm{U}(1)-\mathrm{C}(1) \# 1$ & $49.03(11)$ \\
\hline $\mathrm{C}(2) \# 1-\mathrm{U}(1)-\mathrm{C}(1) \# 1$ & $29.78(11)$ \\
\hline $\mathrm{C}(2)-\mathrm{U}(1)-\mathrm{C}(1) \# 1$ & $151.19(11)$ \\
\hline $\mathrm{C}(1)-\mathrm{U}(1)-\mathrm{C}(1) \# 1$ & $172.93(14)$ \\
\hline
\end{tabular}




\begin{tabular}{|c|c|}
\hline$C(5)-C(1)-C(2)$ & $107.5(3)$ \\
\hline$C(5)-C(1)-C(6)$ & $125.7(3)$ \\
\hline$C(2)-C(1)-C(6)$ & $126.5(3)$ \\
\hline $\mathrm{C}(5)-\mathrm{C}(1)-\mathrm{U}(1)$ & $74.1(2)$ \\
\hline $\mathrm{C}(2)-\mathrm{C}(1)-\mathrm{U}(1)$ & $74.4(2)$ \\
\hline $\mathrm{C}(6)-\mathrm{C}(1)-\mathrm{U}(1)$ & $122.5(2)$ \\
\hline $\mathrm{C}(3)-\mathrm{C}(2)-\mathrm{C}(1)$ & $108.4(3)$ \\
\hline $\mathrm{C}(3)-\mathrm{C}(2)-\mathrm{C}(7)$ & $126.2(4)$ \\
\hline $\mathrm{C}(1)-\mathrm{C}(2)-\mathrm{C}(7)$ & $125.2(4)$ \\
\hline $\mathrm{C}(3)-\mathrm{C}(2)-\mathrm{U}(1)$ & $74.28(19)$ \\
\hline $\mathrm{C}(1)-\mathrm{C}(2)-\mathrm{U}(1)$ & $75.8(2)$ \\
\hline $\mathrm{C}(7)-\mathrm{C}(2)-\mathrm{U}(1)$ & $120.4(2)$ \\
\hline $\mathrm{C}(2)-\mathrm{C}(3)-\mathrm{C}(4)$ & $107.9(3)$ \\
\hline $\mathrm{C}(2)-\mathrm{C}(3)-\mathrm{C}(8)$ & $126.6(4)$ \\
\hline $\mathrm{C}(4)-\mathrm{C}(3)-\mathrm{C}(8)$ & $125.4(4)$ \\
\hline $\mathrm{C}(2)-\mathrm{C}(3)-\mathrm{U}(1)$ & 76.01(19) \\
\hline $\mathrm{C}(4)-\mathrm{C}(3)-\mathrm{U}(1)$ & $75.65(19)$ \\
\hline $\mathrm{C}(8)-\mathrm{C}(3)-\mathrm{U}(1)$ & $116.9(2)$ \\
\hline$C(3)-C(4)-C(5)$ & $107.8(3)$ \\
\hline $\mathrm{C}(3)-\mathrm{C}(4)-\mathrm{C}(9)$ & $125.6(3)$ \\
\hline $\mathrm{C}(5)-\mathrm{C}(4)-\mathrm{C}(9)$ & $125.8(4)$ \\
\hline $\mathrm{C}(3)-\mathrm{C}(4)-\mathrm{U}(1)$ & $74.45(19)$ \\
\hline $\mathrm{C}(5)-\mathrm{C}(4)-\mathrm{U}(1)$ & $74.87(19)$ \\
\hline $\mathrm{C}(9)-\mathrm{C}(4)-\mathrm{U}(1)$ & $124.4(3)$ \\
\hline$C(1)-C(5)-C(4)$ & $108.3(3)$ \\
\hline $\mathrm{C}(1)-\mathrm{C}(5)-\mathrm{C}(10)$ & $125.7(3)$ \\
\hline $\mathrm{C}(4)-\mathrm{C}(5)-\mathrm{C}(10)$ & $125.8(3)$ \\
\hline $\mathrm{C}(1)-\mathrm{C}(5)-\mathrm{U}(1)$ & $76.4(2)$ \\
\hline $\mathrm{C}(4)-\mathrm{C}(5)-\mathrm{U}(1)$ & $75.2(2)$ \\
\hline $\mathrm{C}(10)-\mathrm{C}(5)-\mathrm{U}(1)$ & $118.5(2)$ \\
\hline $\mathrm{N}(1)-\mathrm{C}(11)-\mathrm{C}(12)$ & $122.8(3)$ \\
\hline N(1)-C(11)-C(18) & $120.4(3)$ \\
\hline $\mathrm{C}(12)-\mathrm{C}(11)-\mathrm{C}(18)$ & $116.7(3)$ \\
\hline$C(13)-C(12)-C(17)$ & $117.6(3)$ \\
\hline$C(13)-C(12)-C(11)$ & $122.8(4)$ \\
\hline$C(17)-C(12)-C(11)$ & 119.6(3) \\
\hline
\end{tabular}




$\begin{array}{ll}\mathrm{C}(12)-\mathrm{C}(13)-\mathrm{C}(14) & 121.0(4) \\ \mathrm{C}(15)-\mathrm{C}(14)-\mathrm{C}(13) & 120.1(4) \\ \mathrm{C}(16)-\mathrm{C}(15)-\mathrm{C}(14) & 119.5(4) \\ \mathrm{C}(15)-\mathrm{C}(16)-\mathrm{C}(17) & 120.8(5) \\ \mathrm{C}(16)-\mathrm{C}(17)-\mathrm{C}(12) & 121.0(4) \\ \mathrm{C}(19)-\mathrm{C}(18)-\mathrm{C}(11) & 115.9(3) \\ \mathrm{C}(24)-\mathrm{C}(19)-\mathrm{C}(20) & 118.0(4) \\ \mathrm{C}(24)-\mathrm{C}(19)-\mathrm{C}(18) & 122.5(3) \\ \mathrm{C}(20)-\mathrm{C}(19)-\mathrm{C}(18) & 119.3(4) \\ \mathrm{C}(19)-\mathrm{C}(20)-\mathrm{C}(21) & 120.5(5) \\ \mathrm{C}(22)-\mathrm{C}(21)-\mathrm{C}(20) & 121.1(5) \\ \mathrm{C}(21)-\mathrm{C}(22)-\mathrm{C}(23) & 118.6(4) \\ \mathrm{C}(22)-\mathrm{C}(23)-\mathrm{C}(24) & 120.9(4) \\ \mathrm{C}(23)-\mathrm{C}(24)-\mathrm{C}(19) & 121.0(4)\end{array}$

Symmetry transformations used to generate equivalent atoms:

$\# 1-\mathrm{x}-1, \mathrm{y},-\mathrm{z}+1 / 2$ 
Table S34. Anisotropic displacement parameters $\left(\AA^{2} \times 10^{3}\right)$ for $\left(\mathrm{C}_{5} \mathrm{Me}_{5}\right)_{2} \mathrm{U}[-\mathrm{N}=\mathrm{C}(\mathrm{Ph})(\mathrm{Bz})]_{2}(\mathbf{1 2 b})$. The anisotropic displacement factor exponent takes the form: $-2 \pi^{2}\left[\mathrm{~h}^{2} \mathrm{a}^{* 2} \mathrm{U}^{11}+\ldots+2 \mathrm{hk} \mathrm{a}^{*} \mathrm{~b}^{*} \mathrm{U}^{12}\right]$

\begin{tabular}{|c|c|c|c|c|c|c|}
\hline & $\mathrm{U}^{11}$ & $\mathrm{U}^{22}$ & $\mathrm{U}^{33}$ & $\mathrm{U}^{23}$ & $\mathrm{U}^{13}$ & $\mathrm{U}^{12}$ \\
\hline $\mathrm{N}(1)$ & $35(1)$ & $40(2)$ & $34(2)$ & $5(1)$ & $4(1)$ & 1(1) \\
\hline $\mathrm{U}(1)$ & $26(1)$ & $30(1)$ & $28(1)$ & 0 & $3(1)$ & 0 \\
\hline $\mathrm{C}(1)$ & $34(2)$ & $42(2)$ & $33(2)$ & $5(2)$ & $9(2)$ & $2(2)$ \\
\hline$C(2)$ & $28(2)$ & $47(2)$ & $44(2)$ & $14(2)$ & $13(2)$ & $5(2)$ \\
\hline$C(3)$ & $24(2)$ & $59(2)$ & $38(2)$ & $12(2)$ & $3(2)$ & $-10(2)$ \\
\hline$C(4)$ & $33(2)$ & $41(2)$ & $40(2)$ & $4(2)$ & $7(2)$ & $-10(2)$ \\
\hline$C(5)$ & $32(2)$ & $37(2)$ & $35(2)$ & $8(2)$ & $4(2)$ & $-3(1)$ \\
\hline$C(6)$ & $52(2)$ & $50(2)$ & $38(2)$ & $-2(2)$ & $13(2)$ & $2(2)$ \\
\hline$C(7)$ & $46(2)$ & $69(3)$ & $60(3)$ & $21(2)$ & $20(2)$ & $26(2)$ \\
\hline$C(8)$ & $36(2)$ & $95(4)$ & $48(3)$ & $17(2)$ & $-8(2)$ & $-25(2)$ \\
\hline$C(9)$ & 61(3) & $48(2)$ & $70(3)$ & $-12(2)$ & $7(2)$ & $-22(2)$ \\
\hline$C(10)$ & $51(2)$ & $47(2)$ & $43(2)$ & $15(2)$ & $-2(2)$ & $5(2)$ \\
\hline $\mathrm{C}(11)$ & $36(2)$ & $37(2)$ & $33(2)$ & $3(2)$ & $9(2)$ & $-1(2)$ \\
\hline$C(12)$ & $35(2)$ & $47(2)$ & $33(2)$ & $8(2)$ & $9(2)$ & 1(2) \\
\hline$C(13)$ & $44(2)$ & $56(2)$ & $50(3)$ & $8(2)$ & $6(2)$ & $7(2)$ \\
\hline$C(14)$ & $42(2)$ & $91(4)$ & $62(3)$ & $24(3)$ & $-1(2)$ & $14(2)$ \\
\hline$C(15)$ & $44(2)$ & $101(4)$ & $46(3)$ & $12(3)$ & $-6(2)$ & $-11(2)$ \\
\hline$C(16)$ & $53(2)$ & $73(3)$ & $45(3)$ & $-1(2)$ & $1(2)$ & $-12(2)$ \\
\hline $\mathrm{C}(17)$ & $45(2)$ & $57(2)$ & $39(2)$ & $1(2)$ & $4(2)$ & $-1(2)$ \\
\hline$C(18)$ & $54(2)$ & $45(2)$ & $35(2)$ & $2(2)$ & $8(2)$ & $2(2)$ \\
\hline$C(19)$ & $44(2)$ & $39(2)$ & $34(2)$ & $2(2)$ & $-4(2)$ & $4(2)$ \\
\hline$C(20)$ & $60(3)$ & $43(2)$ & $74(3)$ & $-1(2)$ & $14(2)$ & $6(2)$ \\
\hline$C(21)$ & $81(3)$ & $42(2)$ & $81(4)$ & $2(2)$ & $0(3)$ & $-2(2)$ \\
\hline $\mathrm{C}(22)$ & $63(3)$ & $59(3)$ & $52(3)$ & $10(2)$ & $-6(2)$ & $-15(2)$ \\
\hline$C(23)$ & $53(2)$ & $75(3)$ & $46(3)$ & $0(2)$ & $10(2)$ & $-5(2)$ \\
\hline$C(24)$ & $55(2)$ & $45(2)$ & $46(2)$ & $-3(2)$ & $8(2)$ & $3(2)$ \\
\hline
\end{tabular}


Table S35. Hydrogen coordinates ( x 104) and isotropic displacement parameters $\left(\AA^{2} \times 10^{3}\right)$ for $\left(\mathrm{C}_{5} \mathrm{Me}_{5}\right)_{2} \mathrm{U}[-\mathrm{N}=\mathrm{C}(\mathrm{Ph})(\mathrm{Bz})]_{2}$ (12b).

\begin{tabular}{|c|c|c|c|c|}
\hline & $\mathrm{x}$ & $\mathrm{y}$ & z & $\mathrm{U}(\mathrm{eq})$ \\
\hline $\mathrm{H}(6 \mathrm{~A})$ & -4800 & 1464 & 388 & 70 \\
\hline $\mathrm{H}(6 \mathrm{~B})$ & -4562 & 482 & 797 & 70 \\
\hline $\mathrm{H}(6 \mathrm{C})$ & -3844 & 1035 & 408 & 70 \\
\hline $\mathrm{H}(7 \mathrm{~A})$ & -3144 & 77 & 1524 & 86 \\
\hline $\mathrm{H}(7 \mathrm{~B})$ & -2999 & 184 & 2334 & 86 \\
\hline $\mathrm{H}(7 \mathrm{C})$ & -2324 & 655 & 1860 & 86 \\
\hline $\mathrm{H}(8 \mathrm{~A})$ & -2754 & 2962 & 3178 & 91 \\
\hline $\mathrm{H}(8 \mathrm{~B})$ & -2118 & 2228 & 2838 & 91 \\
\hline $\mathrm{H}(8 \mathrm{C})$ & -2845 & 1814 & 3288 & 91 \\
\hline $\mathrm{H}(9 \mathrm{~A})$ & -3507 & 4130 & 2746 & 89 \\
\hline $\mathrm{H}(9 \mathrm{~B})$ & -4461 & 4299 & 2400 & 89 \\
\hline $\mathrm{H}(9 \mathrm{C})$ & -3648 & 4515 & 1978 & 89 \\
\hline $\mathrm{H}(10 \mathrm{~A})$ & -5247 & 2883 & 599 & 71 \\
\hline $\mathrm{H}(10 \mathrm{~B})$ & -4733 & 3857 & 815 & 71 \\
\hline $\mathrm{H}(10 \mathrm{C})$ & -5498 & 3494 & 1245 & 71 \\
\hline $\mathrm{H}(13)$ & -2674 & -1510 & 4043 & 60 \\
\hline $\mathrm{H}(14)$ & -1664 & -1359 & 4999 & 78 \\
\hline $\mathrm{H}(15)$ & -1535 & 125 & 5605 & 77 \\
\hline $\mathrm{H}(16)$ & -2425 & 1438 & 5261 & 69 \\
\hline $\mathrm{H}(17)$ & -3449 & 1292 & 4330 & 56 \\
\hline $\mathrm{H}(18 \mathrm{~A})$ & -4175 & -1068 & 2601 & 54 \\
\hline $\mathrm{H}(18 \mathrm{~B})$ & -3319 & -1442 & 3022 & 54 \\
\hline $\mathrm{H}(20)$ & -3784 & -3131 & 3069 & 70 \\
\hline $\mathrm{H}(21)$ & -4638 & -4342 & 3521 & 82 \\
\hline $\mathrm{H}(22)$ & -5802 & -3924 & 4142 & 70 \\
\hline $\mathrm{H}(23)$ & -6106 & -2271 & 4312 & 69 \\
\hline $\mathrm{H}(24)$ & -5269 & -1057 & 3861 & 58 \\
\hline
\end{tabular}

